\title{
Vegetation diversity of the early Miocene St. Bathans Palaeovalley Ecosystem, New Zealand
}

\author{
Mike Pole
}

\begin{abstract}
The St. Bathans Member is the stratigraphically lowest unit of the Manuherikia Group, New Zealand. It consists of braided river facies, which accumulated within a 'St. Bathans Palaeovalley' that was incised into metamorphic basement. Incision probably resulted from gentle uplift to the west in the late Oligocene and may have also initiated deltaic sedimentation in the Eastern Southland Lignite Basin. The St. Bathans Member dates to the early Miocene, and its predominant fill of sand and gravel also contains muddy units that are rich in well-preserved plant fossils.

The regional vegetation was rainforest of very high diversity, with 145 angiosperms 14 conifers, and two cycad-like gymnosperms currently known from leaf cuticle, compressions and shoots, and more taxa known only from the palynological record. The vegetation was dominated by a mixture of conifers (mostly Podocarpaceae, especially Retrophyllum, but also Araucariaceae, Cupressaceae and Taxaceae) and angiosperms that included large-leaved Nothofagus, Lauraceae, Myrtaceae Proteaceae, and Elaeocarpaceae, and/or Cunoniaceae. The mean annual temperature was around $14-20^{\circ} \mathrm{C}$, and the broader climate condition was warm-temperate/mesothermal/paratropical, ever-wet and entirely fire-free.

In this paper 13 new leaf parataxa are described. Of these, MANU-46 and MANU61 probably belong to the Sapindaceae, MANU-50 is identified as Strychnos (Loganiaceae, the first record of the genus from New Zealand), and MANU-53 to the Myrtaceae. MANU-57 belongs in the Proteaceae, having cuticle with similarities to Musgravea, however, the leaf architecture suggests it may have been produced by another genus. MANU-58 represents another Proteaceae, genus uncertain. A previously described leaf parataxon, MANU-15, probably belongs in the Cunoniaceae.
\end{abstract}

Mike Pole. Queensland Herbarium, Brisbane Botanic Gardens Mt Coot-tha, Toowong, Qld, Australia. murihiku@yahoo.com

Keywords: Miocene; cuticle; paleoclimate; rainforest; Manuherikia

Submission: 3 June 2019. Acceptance: 4 November 2019.

Pole, Mike. 2019. Vegetation diversity of the early Miocene St. Bathans Palaeovalley Ecosystem, New Zealand. Palaeontologia Electronica 22.3.75. 1-50. https://doi.org/10.26879/1004

palaeo-electronica.org/content/2019/2848-early-miocene-st-bathans-paleo

Copyright: November 2019 Paleontological Society.

This is an open access article distributed under the terms of Attribution-NonCommercial-ShareAlike 4.0 International (CC BY-NC-SA 4.0), which permits users to copy and redistribute the material in any medium or format, provided it is not used for commercial purposes and the original author and source are credited, with indications if any changes are made.

creativecommons.org/licenses/by-nc-sa/4.0/ 


\section{INTRODUCTION}

The Manuherikia Group is an extensive fluviolacustrine unit (Douglas, 1986) of Miocene age (Mildenhall and Pocknall, 1989) in southern New Zealand. Douglas (1986) concluded that the oldest sediments were deposited by braided rivers flowing in valleys incised into the basement rock (schist and greywacke), and he termed these the St. Bathans Member of the Dunstan Formation. Study of outcrop and drillcore established that the palaeovalleys had a general west-east orientation, and that flow was to the east or southeast. It is not clear where on the coast the rivers flowed to, although it has been suggested that gravels in the marginal marine Gore Lignite Measures in Southland, may be the distal equivalent of these rivers (Issac et al., 1990). As the incised valley filled with sediment, and sedimentation spread out over a much broader area, the fluvial style changed to meandering.

The St. Bathans Member (SBM, exposed near the village of St. Bathans, Figure 1) has been a rich source of organically-preserved (with cuticle) plant fossils. These include an extinct gymnosperm (Hill and Pole, 1994), a rich conifer flora (Pole, 1992, 1997, 2007a), a diverse array of Lauraceae dispersed cuticle and mummified leaves (Pole, 1993a, 2007b), a new species of Nothofagus, N. azureus (Pole 1993b), Proteaceae (Pole, 1998), Myrtaceae (Pole et al., 2008), the existence of some plant taxa now restricted to New Caledonia (Pole, 2010), a flower (Pole et al., 2003) and a wide range of other angiosperm leaf cuticle taxa including monocots and monocot seeds, (Pole, 2007c, 2008) and mummified leaves (Pole, 1993c). Samples from the St. Bathans Member were part of a quantitative

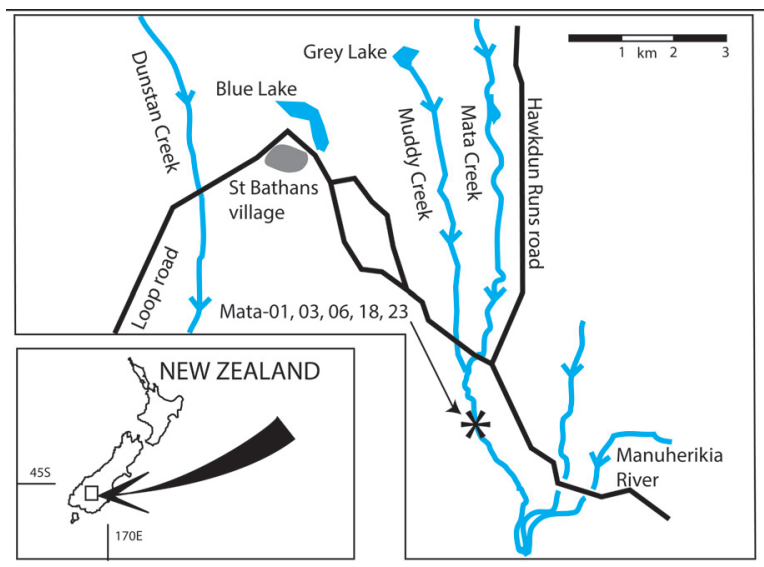

FIGURE 1. General locality map showing the position of St. Bathans in New Zealand (bottom left) and the major localities of Blue Lake, Grey Lake, and Mata Creek. palynological zonation (Pole and Douglas, 1998), a reconstruction of Miocene atmospheric dynamics in New Zealand (Pole, 2003), and an assessment of Neogene temperatures (Pole, 2014). Reichgelt et al. (2015, p. 351) report what they called "a newly discovered mudstone lens." This particular lens (GL-05) has had its precise grid reference and dispersed cuticle details in four previous publications (Pole, 2007a, 2007b, 2008, 2014).

Most of the angiosperm material has been finely fragmented (as a result of fluvial reworking), necessitating it being treated as dispersed cuticle. Conifer shoots are typically small and robust enough to survive the redeposition. This kind of material formed the focus of the earliest phase of research. However, several locations were discovered in which intact, or mostly so, angiosperm leaves were present. These have been accumulated while experimenting with new techniques to deal with them.

This current paper describes further intact angiosperm leaves, documents some of the palynology, and integrates these with dispersed cuticle into the local stratigraphy for an enhanced understanding of what was the 'St. Bathans Palaeovalley Ecosystem'.

\section{MATERIALS AND METHODS}

The main outcrops of the St. Bathans Member occur at Blue Lake (Figure 2), and what is locally known as Grey Lake (Figure 3), both near the village of St. Bathans. There are also outcrops present along Mata $\mathrm{Ck}$ a few kilometres distant. It is also well-represented in a series of coal-exploration bore holes across the wider region (Douglas, 1986).

The basic unit of collection is termed a 'sample' and in most cases this was a plastic bag of mudstone (c. 100-200 g), which is adequate for dispersed cuticle analysis. Samples are prefixed with "BL-“", "GL-" and "Mata-". In cases where macrofossils are common, repeat collections were made from the same mudstone unit, sometimes extending laterally for 4 or $5 \mathrm{~m}$. These larger collections are still covered by the same sample number.

The appearance of intact fossil leaves exposed on mudstone varies from a golden brown, with venation details apparent, to more commonly opaque black cuticular material that obscures details. Leaves were treated in a variety of ways. Some were left exposed, while in some cases they could be floated off the matrix and then mounted between glass or plastic sheets using glycerine 


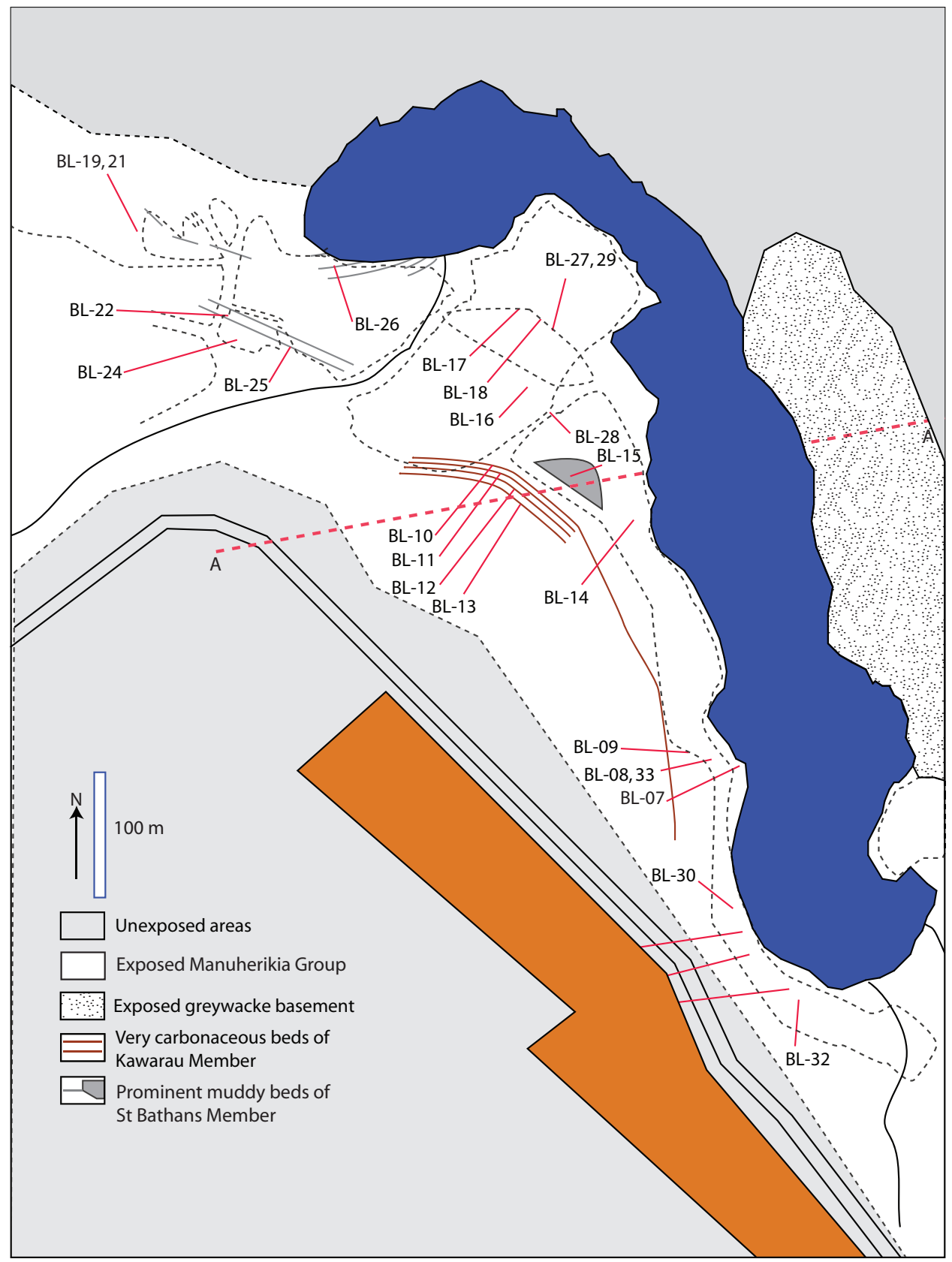

FIGURE 2. Blue Lake, St. Bathans. Showing location of samples. Greyed area is soil/no-outcrop area. Dashed lines show steep cliffs or scarps, usually a result of gold-sluicing activity. A-A' is the line of cross-section shown in Figure 4.

jelly. Some leaves were first coated with Polyvinyl Butryl Resin (PVB). The mudstone was then disaggregated in hot water and hydrogen peroxide. This left the leaf attached to a resin 'skin' that could be further cleaned with hydrogen peroxide and hydrofluoric acid and then mounted between plastic using glycerine jelly. Disaggregated sediment can be sieved to concentrate the finer plant remains and scanned under binocular microscope and items of interest retrieved with tweezers. Following this, leaf material can be further processed to clean dispersed cuticle by prolonged warming in concentrated hydrogen peroxide, stained in safranin, and mounted in glycerine jelly on microscope slides, or air-dried and gold or platinum coated on electron microscope stubs.

Specimens and microscope slides are prefixed with "SL", "SB" or "LX". Scanning Electron Microscope (SEM) stubs are prefixed with "S-". 


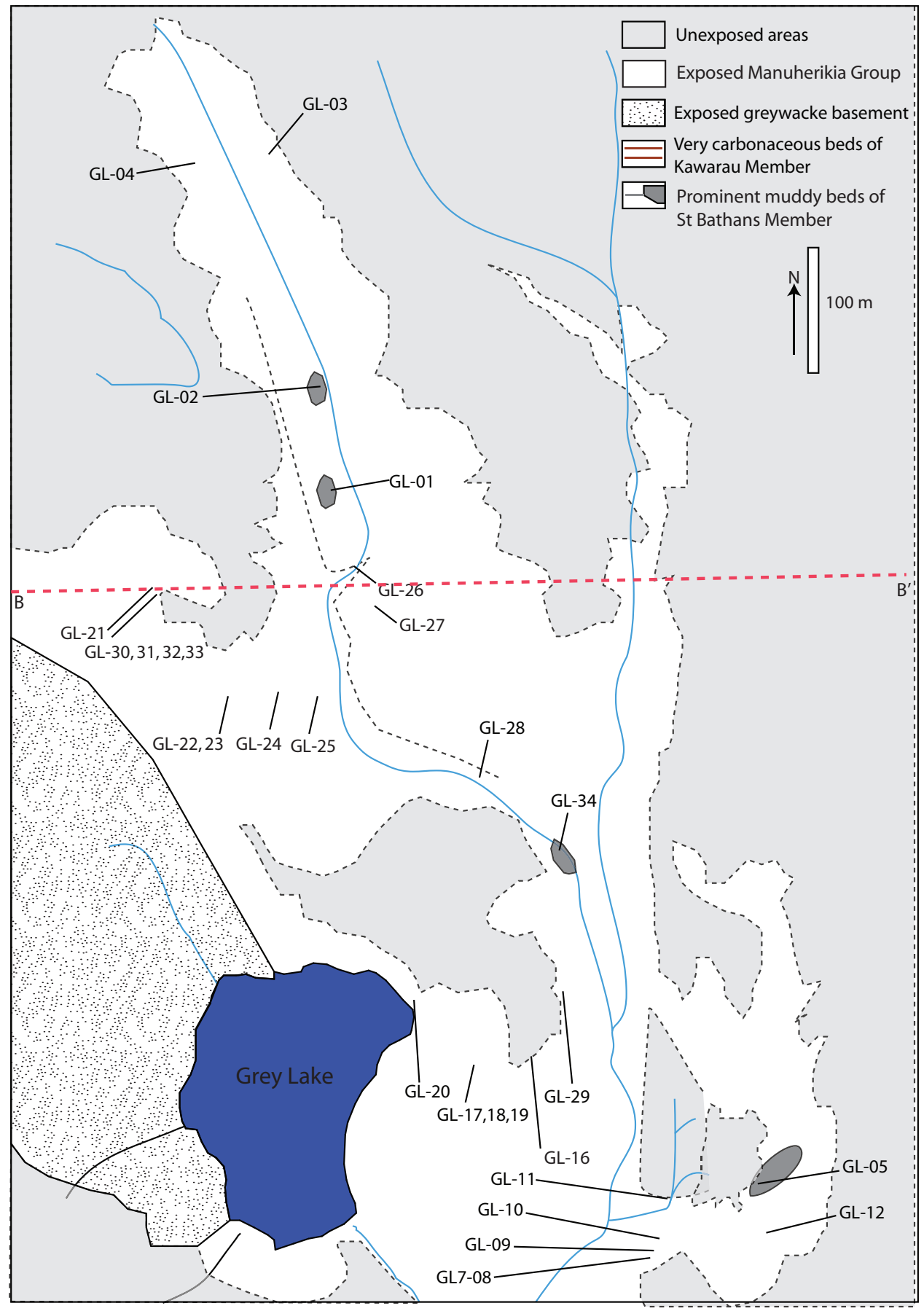

FIGURE 3. Grey Lake, to the north of St. Bathans. Showing location of samples. Greyed area is soil/no-outcrop area. Dashed lines show steep cliffs or scarps, usually a result of gold-sluicing activity. B-B' is the line of cross-section shown in Figure 4.

Leaf images were processed using Adobe Photoshop. Pixels were deleted in some figures to remove the background surrounding the rocks and in some glass/plastic/glycerine leaf mounts where there was some discolouration of glycerine external to the leaf.
The dispersed cuticle data were explored using PAST (Hammer et al., 2001). The most appropriate analyses were considered to be nonmetric multidimensional scaling (NMDS) with the Dice similarity measure.

Palynological preparations were made by removing silica in hydrofluoric acid, followed by 


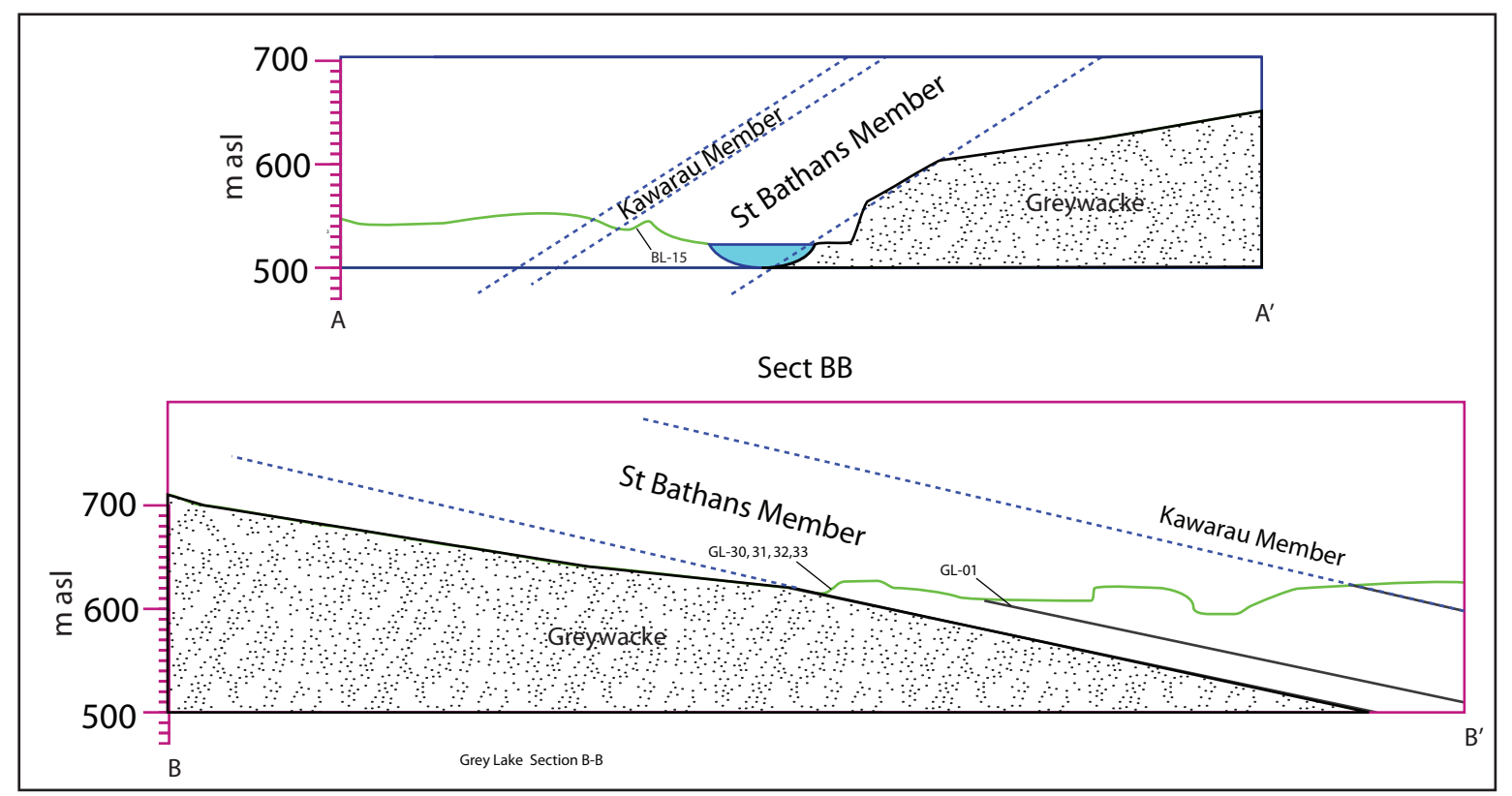

FIGURE 4. Representative cross-sections of Blue and Grey Lakes. The inferred earlier dip and extension of the St. Bathans and Kawarau Members (before erosion and mining) is shown by dashed lines.

warm nitric acid, $\mathrm{KOH}$, staining in safranin and mounting in glycerine jelly. Palynological taxa follow Raine et al. (2011). Further illustrations of Manuherikia Group pollen and spore taxa can be seen in Pocknall and Mildenhall (1984), Mildenhall and Pocknall (1989) and Mildenhall (1989).

Material is housed in the Department of Geology, Auckland University.

\section{GEOLOGICAL BACKGROUND}

Blue Lake and Grey Lake are situated on the southern and northern limbs respectively, of an antiform of Mesozoic greywacke basement (Figure 4). Greywacke basement is exposed along the east-northeastern edge of Blue Lake. The basement-sediment contact dips to the west-southeast at around $30-40^{\circ}$. At Grey Lake the greywacke basement is exposed along the west-southwestern edge and the basement-sediment contact dips east-northeast at around $12^{\circ}$. The quartz-gravel rich SBM directly overlies the greywacke basement and is itself overlain by the cyclic clay-rich, carbonaceous muds of the Cromwell Submember of the Dunstan Member. This unit is visible in the northwest of Blue Lake (where it is overlain by greenish lacustrine muds of the Ewing Member and Bannockburn Formation) and in the far northeast of Grey Lake. St. Bathans Member is also present along Mata Ck, where it occurs with Ewing and Fiddlers Members, although this is highly faulted and slumped, and the broader structure is unclear.

Douglas (1986) interpreted the SBM as mostly representing the deposits of bars of quartzose gravel that formed within a braided river system that changed in character over time. Within or between the gravel units are muddy units, either lensoidal or more sheet-like. These were deposited from suspension after floods in a variety of local environments, from flood-basins, swamps and abandoned channels. Douglas (1986) documented four sequences from Grey Lake (A-D). Reichgelt et al. (2015) claimed that the GL-05 deposit was ( $p$. 351) "probably deposited in an oxbow lake", ( $p$. 362) "growing on delta plain", and (throughout) peripheral to a "Lake Manuherikia". These comments have no published basis and contradict the braided river interpretation of Douglas (1986 - the only sedimentological work done on the SBM). The incised valley-fill of the SBM occurs well-below the late-stage sediments of 'Lake Manuherikia'. Reichgelt et al. (2015) furthermore provided a stratigraphic section (their fig. 2) indicating the GL-05 deposit approximately $5 \mathrm{~m}$ above basement. The deposit lies approximately $250 \mathrm{~m}$ NE of basement dipping to the NE, and is clearly tens of meters (c. $80 \mathrm{~m}$ ) above basement. 

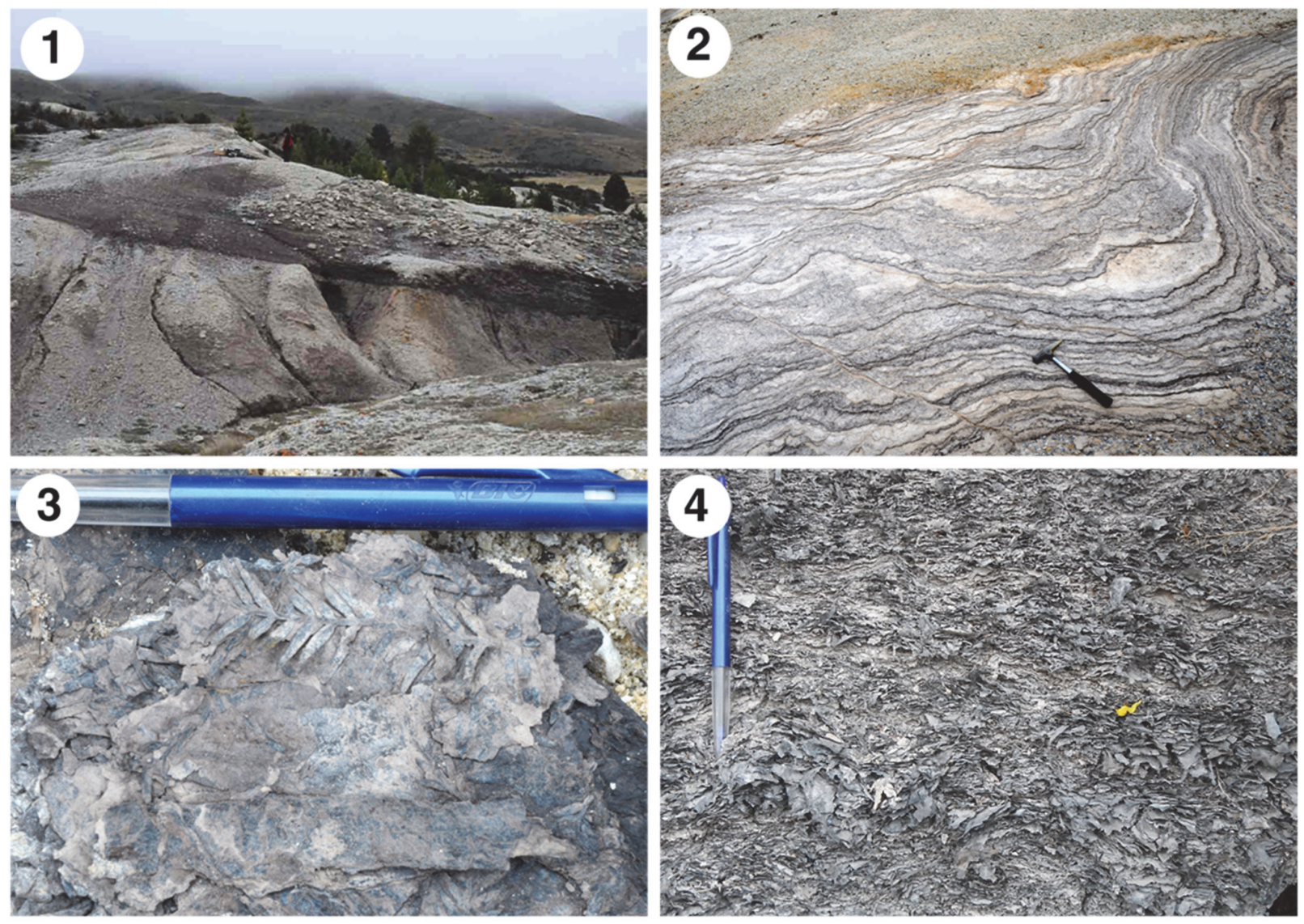

FIGURE 5. Four important plant-fossil bearing facies in the St. Bathans Palaeovalley. 1. Muddy lenses (GL-02, figure near top of bed, gives scale). 2. Beds of redeposited leaf cuticle (GL-24, rock hammer gives scale). 3. In situ deposition leaf packs. Note intact Retrophyllum shoot (GL-32, ball-point pen gives scale). 4. Redeposited leaf packs (Mata01, ball-point pen gives scale).

\section{RESULTS}

Carbonaceous muddy units with plant fossils fall into at least four facies (Figure 5):

1. Muddy lenses that are mostly almost massive but with a crude fissility and contain abundant angiosperm leaf material ranging from fragments to intact leaves and occasional pieces of very compressed wood. The lenses are often around 1-3 m thick, usually indistinctly bedded, typically with a sharp upper and lower contacts with quartz gravel, often truncated laterally by gravel-filled channels and often fill abandoned channels. These are interpreted as primary flood material filling abandoned channels or similar depressions on the valley (see Douglas, 1986, for details). The homogenous lenses may have filled from a single flood, while others with more stratified fill may have accumulated over a longer time from periodic floods. This facies includes:
GL-01, -02. Two patches of very carbonaceous mud in the north of Grey Lake that probably represent the same stratigraphic unit.

GL-03. Exposed in the eastern wall of the Grey Lake area. Blocks of mud often tumble down the slope making collecting relatively easy. In the field the assemblage is notable for its common Proteaceae leaves.

GL-05. With common leaf fossils, at the southeastern margin of the Grey Lake area.

BL-30. A bed in the cliffs edging Blue Lake. Blocks of mudstone are continuously falling into the lake. Leaves of Nothofagus azureus are a common component of the assemblage as seen in the field, as are occasional masses of Podocarpus alwyniae Pole (1992) and palm fronds.

2. Beds of finely comminuted leaf material (often small fragments of cuticle) in mud. These are interpreted as being redeposited primary flood deposits where currents have winnowed and size-sorted 
partially decomposed leaves. Typical examples of this facies are samples GL-22, 23.

3. Highly carbonacaeous units with 'mats' of leaves and/or wood. These are interpreted as almost in-situ build-up of plant material in standing water. The facies includes:

GL-34. Numerous fragments of lignified wood that have washed out over the south-eastern corner of Grey Lake all appear to come from an exposure of such a facies in the bed of a small stream. This is likely to represent an almost in-situ accumulation of plant material.

GL-30, 31, 32, 33. These samples come from a location that is unique in that a thin-bedded, noncarboniferous, laminated mud unit directly overlies cross-bedded gravel. The lower $40 \mathrm{~cm}$ of bedded mud has only sparse and small plant fragments. The carbonaceous content, including plant fragments, increases steadily upwards. Intact leaves on mud bedding surfaces are common about a meter above base, and then at the top it becomes a litter of predominantly, Retrophyllum shoots. A few logs are also present. The unit is then overlain by more sand and gravel.

The short sequence is interpreted as a lake that formed in an abandoned channel. Periodic floodwaters filled in the channel until Retrophyllum swamp forest was established and appears to have grown over what remained of the lake.

Material that represents soil is rare. In-situ lignite is essentially absent from the Blue and Grey Lake area, although it can be present as 'rip-up' clasts in channels (e.g., Douglas, 1986). A very carbonaceous to almost lignitic unit at Blue Lake contains resin lumps and may be an in-situ swamp accumulation. Over the SBM in general, no large in situ stumps are known, although zones of roots are locally present (Douglas, 1986). The general dearth of soils might reflect the highly unstable environment in which sediments were often reworked. In addition, no shells or bones have been noted.

4. Deposited leaf mats. An exposure in Mata Ck (Mata-01) is virtually entirely of compressed leaves. It is a leaf mat but appears different from leaf mats included in Facies 3in that it is wellsorted, with almost no sediment or wood. It is comparable to leaf mats that accumulate on point bars of some forested tropical rivers (the author has observed thick mats of leaves deposited along Indonesian rivers). It is therefore interpreted as a deposited leaf mat rather than an in situ accumulation.
Although many beds within the SBM are clearly lens-like or channelled, and therefore are not continuous for any great distance, some units are more extensive. In particular a prominent zone about 3-4 $m$ thick of very pale well sorted very fine sand, grading up to a massive mud or clay extends over most of Grey Lake. It is a useful local marker horizon, possibly lacustrine although does not have plant macrofossils.

Leaf-size histograms for GL-01 are shown in Figure 6. Leaf parataxa and cuticle descriptions are presented as Systematic Palaeontology, in family order following LAPG III (Haston et al., 2009). Leaf architecture terms follow Hickey (1973), Dilcher (1974), Pole (1991), and Ellis et al. (2009). Dispersed cuticle data were recorded as presenceabsence of taxa and presented in Table 1 (Gymnosperms), Table 2 (Monocots), Table 3 (Lauraceae), Table 4 (Myrtaceae), Table 5 (Proteaceae) and Table 6 (all other angiosperms. Leaf parataxon distribution is given in Table 7. Palynological data are presented in Table 8.

\section{Macrofossils and Dispersed Cuticle}

Exploration of the presence-absence dispersed cuticle data (Tables 1-6) set by clustering analysis utilising the Dice (Sorensen), Jaccard, Kulczynski, Ochiai and Raup-Crick indices and the Paired group and Single linkage algorithms did not produce any robust clusters or ordinations (and are therefore not illustrated here). This is interpreted as resulting from the data-set being relatively homogenous to start with (i.e., derived from a single 'flora', although different successional stages likely occurred) and with many of the samples being redeposited - and perhaps mixtures of earlier mud lenses.

\section{Gymnosperms}

Conifers are an important component of the SBM as a whole (Table 1, present in $72 \%$ of all samples) but conspicuous by their absence in some samples. There is no obvious correlation of conifer diversity with overall sample diversity. For example, GL-01 has 23 angiosperm taxa and seven conifers, while GL-02 has 25 angiosperm taxa and no conifers. Retrophyllum is the most widespread conifer, and if a sample has only one conifer it is usually Retrophyllum. Dacrycarpus is the second. Other conifer genera include Acmopyle, Araucaria, Dacrydium, Kakahuia, Lepidothamnus, Papuacedrus, Podocarpus, Prumnopitys and an indeterminate Taxaceae (Pole, 1997, 2007a). At least three other gymnosperms were 

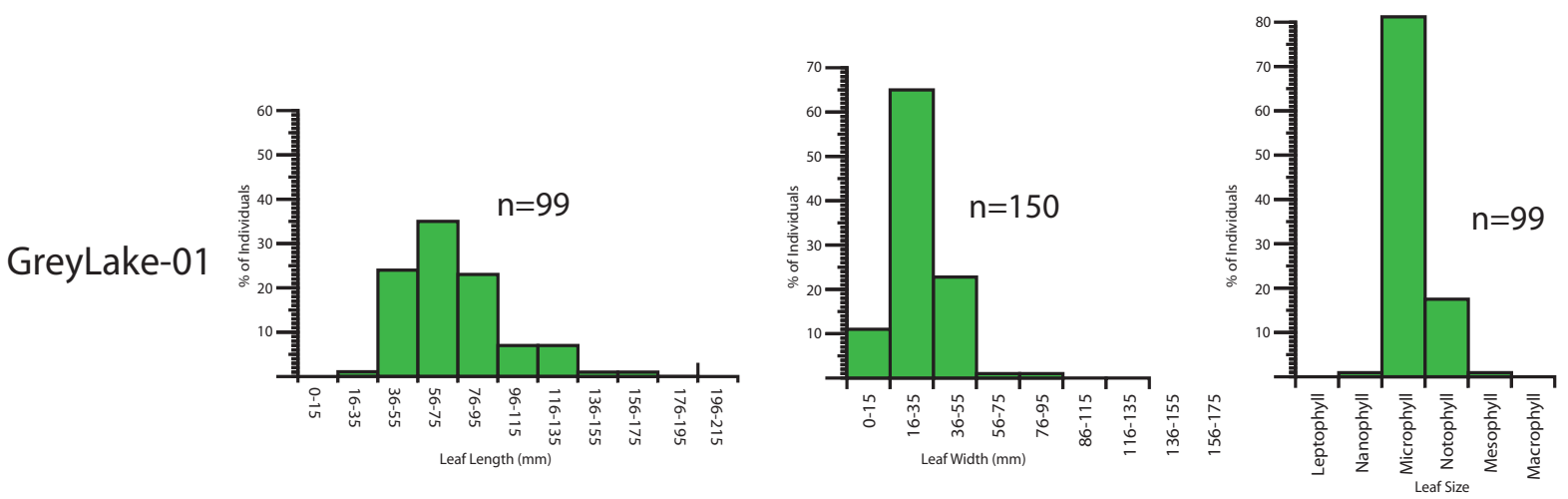

FIGURE 6. GL-01 leaf size histograms, showing length (left), width (middle) and the leaf size classes of Webb (1959) (right). The average leaf length is $\mathrm{c} .75 \mathrm{~mm}$.

present; a genus of the Gnetalaceae (Pole, 2008), and two cycad or cycad-like taxa- Pterostoma (Hill and Pole, 1994) and an un-named genus (Pole, 2007a).

Some conifer taxa that were likely in New Zealand at the time are notable by their absence or rarity in the St. Bathans Palaeovalley; for example Araucaria is known from only a few scattered leaf fragments (Pole, 2007a) while undoubted Agathis are unknown. This is consistent with Araucariaceae pollen being rare. Phyllocladus pollen is widespread although unknown as macrofossils. Their absence or rarity in the SBM is probably due to factors such as the absence of peat-based soils.

\section{Monocots}

Palm fronds are present at BL-30 (Table 2) and probable palm dispersed cuticle is also present while palm pollen is ubiquitous. This record is interpreted as palms scattered through forest vegetation, contrasting with the overlying Kawarau Member in which there were palm-dominated swamps (Pole and Douglas, 1998; Pole, 2003).

Typha, which is present as cuticle in GL-01, and Typha seeds are known in BL-31 and GL-11 (Pole, 2007). In general Typha is a primary colonist after disturbance and requires relatively nutrientrich sites (e.g., Wehi and Clarkson, 2007) such as found in a frequently disturbed braided river habitat.

Monocots notable by their apparent absence from the SBM include Ripogonum, known as impressions and dispersed cuticle in the Kawarau Member at Bannockburn (Pole, 1993d). This is odd as it is an easily recognisable leaf, and the cuticle is robust. Phormium might also be expected but has also not been found. Its pollen is distinctive although tends to be rare (under-represented).

\section{Other Angiosperms}

The most important angiosperm groups in the SBM dispersed cuticle record are Lauraceae (Table 3), occurring in $90 \%$ of all samples, and including Beilschmiedia, Cryptocarya, Endiandra and Litsea (Pole, 2007b), Myrtaceae (Table 4) in 59 $\%$ of all samples, including Metrosideros and probably a Syzygium (Pole et al., 2008), and Proteaceae (Table 5) in $67 \%$ of all samples. These families are also prominent in the intact, leaf parataxa record (Table 7). Casuarinaceae pollen is ubiquitous (Table 8) although not common, but Gymnostoma macrofossils are rare. Other families that were present include Cunoniaceae/Elaeocarpaceae (in addition to leaf material, there are flowers, possibly of Acsmithia, Cunoniaceae, Pole et al., 2003), Winteraceae, Atherospermataceae, Monimiaceae (including Hedycarya), Menispermaceae (cf. Pleiogyne), Santalaceae (Notothixos), Strasburgeriaceae (Strasburgeria), Meliaceae, Sapindaceae (cf. Cupaniopsis), Myrsinaceae (cf. Ardisia), Grisseliniaceae (Grisselinia) and Argophyllaceae (Argophyllum) (Pole, 2008).

Broad differences in floristic composition are apparent in the field. For example, the large-leaved Nothofagus azureus Pole (1993b) is a prominent component of BL-30. In GL-03, both Nothofagus azureus and the compound-leaved Proteaceae MANU-58, are co-dominant, but both are rare in GL-05. However, there is no straight-forward way to quantify these results, as apart from the few easily identifiable taxa, most remains are fragmentary, or need cuticular details to confirm identification. Note that despite being a common leaf macrofossil, Nothofagus azureus has very thin cuticle and does not form a part of the dispersed cuticle assemblages. The combination of extraordinary 
TABLE 1. Presence-absence matrix of St. Bathans Member conifers.

\begin{tabular}{|c|c|c|c|c|c|c|c|c|c|c|c|c|c|c|c|c|c|c|c|c|c|c|c|c|c|c|c|c|c|c|c|c|c|c|c|c|c|}
\hline TAXON & 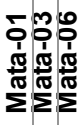 & 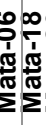 & & & & & m. & & & & & & & & พิ่ & 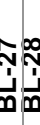 & & & & & 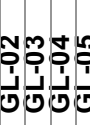 & 1َ. & 象 & & & Ne & & & & & & & & & & & \\
\hline Acmopyle & 1 & & 1 & & & & & & & & & & & & & & & & 1 & 1 & 1 & & & & & & 1 & & 1 & & & & 1 & & & & \\
\hline Araucariaceae & & & & & & & & & & & & & & & & & & & & 1 & & & & & & & 1 & & & 1 & 1 & & & & & & \\
\hline Agathis-like & & & & & & & & & & & & & & & & & & & & 1 & & & & & 1 & & & & & & & & & & & & \\
\hline Dacrycarpus & 1 & & & & 1 & 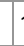 & 1 & 1 & & & & 1 & 1 & 1 & 1 & & 1 & & \begin{tabular}{l|l}
1 & 1
\end{tabular} & \begin{tabular}{|l|l|}
1 & 1
\end{tabular} & 1 & & & & & 1 & & 11 & \begin{tabular}{|l|l|}
1 & 1
\end{tabular} & & \begin{tabular}{l|l}
1 & 1
\end{tabular} & 1 & 1 & 1 & 1 & & 1 \\
\hline Dacrydium & & & & & & & & & & & & & & & & & 1 & & 1 & & & & & & & & & & & & & & & & & & \\
\hline Greylackia & & & & & 1 & & & & & & & & & & & & & & & & & & 1 & & & & 1 & & & & & & & 1 & & & \\
\hline Lepidothamnus & 1 & & & & & & & & & & & & & & & & & & & & & & & & & & & & & 1 & & & & & & & \\
\hline Papuacedrus & & & & & & & & & & & & & & & & & 1 & & & & & & & & & & & & & & & & & & & & \\
\hline $\begin{array}{l}\text { Podocarpus } \\
\text { alwyniae }\end{array}$ & & & & & & & & & & & & & & & & & 1 & & & & & & & & & & & & & & & & & & & & \\
\hline $\begin{array}{l}\text { Podocarpus sp. } \\
\text { "Mata Ck" }\end{array}$ & 1 & & & & & & & & & & & & & & & & & & 111 & 11 & 1 & & & & 1 & & & & & & & & 1 & 1 & 1 & & \\
\hline $\begin{array}{l}\text { Podocarpus sp. } \\
\text { "sinuous" }\end{array}$ & & & & & & & & & & & & & & & & & & & & & & & & & & & & & & & & & & & 1 & & \\
\hline $\begin{array}{l}\text { Prumnopitys } \\
\text { taxifolia }\end{array}$ & 11 & 1 & & & & & 1 & & & 1 & & & & & & & & & 1 & 1 & 1 & & & & & & & & $?$ & & & & & 1 & & & \\
\hline Kakahuia & 1 & & & & & & & & & & & & & & & & & & & 1 & & & & & & & & & & & & & & & & & \\
\hline Pterostoma & & & 1 & & 1 & & & & & & & & & & & & & & 1 & & 11 & 11 & \begin{tabular}{l|l}
1 \\
1
\end{tabular} & & 1 & & & 1 & 1 & 1 & 1 & & & & & & \\
\hline Retrophyllum & \begin{tabular}{l|l}
1 & 1
\end{tabular} & \begin{tabular}{l|l}
1 & 1
\end{tabular} & 1 & 1 & \begin{tabular}{|l|l|}
1 & 1
\end{tabular} & & 1 & 1 & & 1 & 1 & 1 . & 1 & & 1 & & & 1 & 1 & & & 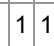 & 1 & & \begin{tabular}{l|l}
1 & 1
\end{tabular} & 1 & & 11 & \begin{tabular}{|l|l|}
1 & 1
\end{tabular} & 1 & \begin{tabular}{l|l}
1 & 1
\end{tabular} & 1 & 1 & 1 & & & 1 \\
\hline Taxaceae & 1 & & & & & & & & & & & & & & & & & & & & & & & & & & & & & & & & & & & & \\
\hline
\end{tabular}

TABLE 2. Presence-absence matrix of St. Bathans Member monocot dispersed cuticle taxa.

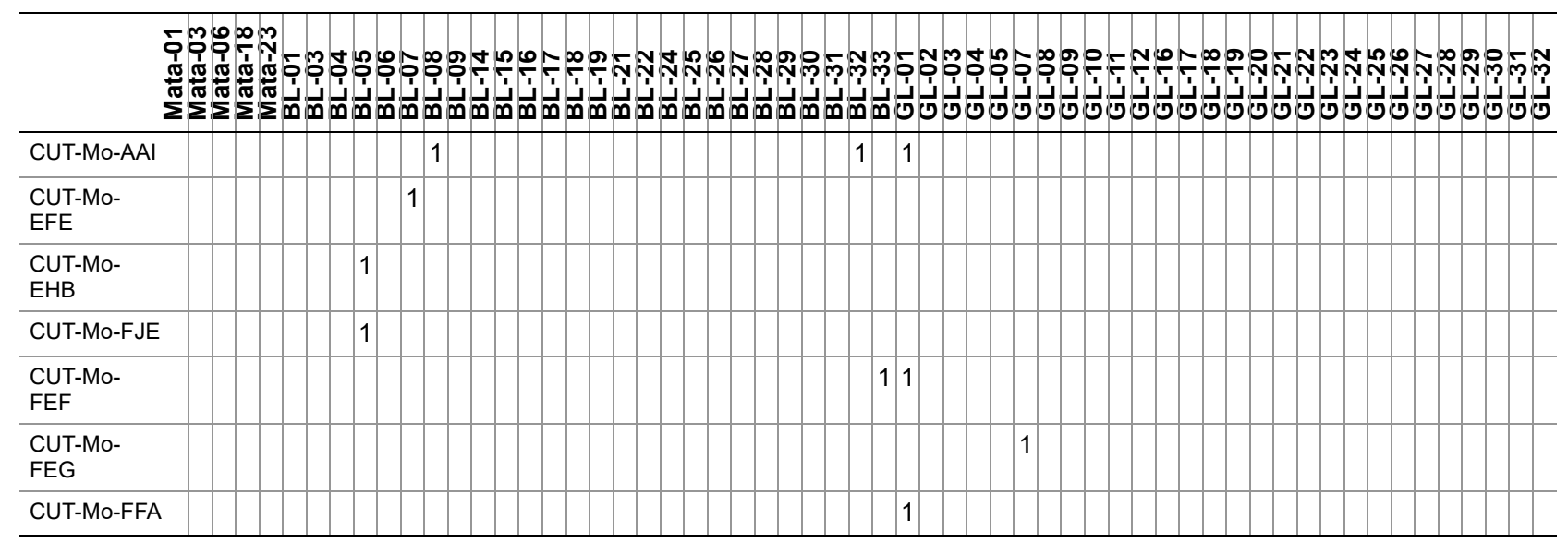

large size and very thin cuticle suggest that Nothofagus azureus was deciduous (Pole, 2014).

The floristic composition at the family level the predominance of Podocarpaceae, Elaeocarpaceae/Cunoniaceae, Lauraceae, Myrtaceae, is similar to New Zealand today. It is also typical of 'montane' rainforests in the tropics (Paijmans, 1976; Whitmore, 1986). Lauraceae and Myrtaceae tend to be more prominent in lower montane forests and conifers in upper montane forests. The classification is a structural one, but a possible relationship with cloudiness has been noted (e.g., Whitmore, 1986).

\section{Palynology}

Most samples (Table 8) are dominated by Nothofagidites cranwelliae (Couper, 1953) Mildenhall and Pocknall (1989) (Nothofagus s.g. Brassospora), and most also contain N. lachlanae (Couper, 1953) Mildenhall and Pocknall (1989) (Nothofagus s.g. Fuscospora) and N. asperus (Cookson, 1959) Romero (1973) (Nothofagus s.g. Lophozonia). N. falcatus (Cookson, 1959) Hekel (1972) and $N$. 
Pole: Early miocene St. Bathans Paleo

TABLE 3. Presence-absence matrix of St. Bathans Member Lauraceae dispersed cuticle taxa.

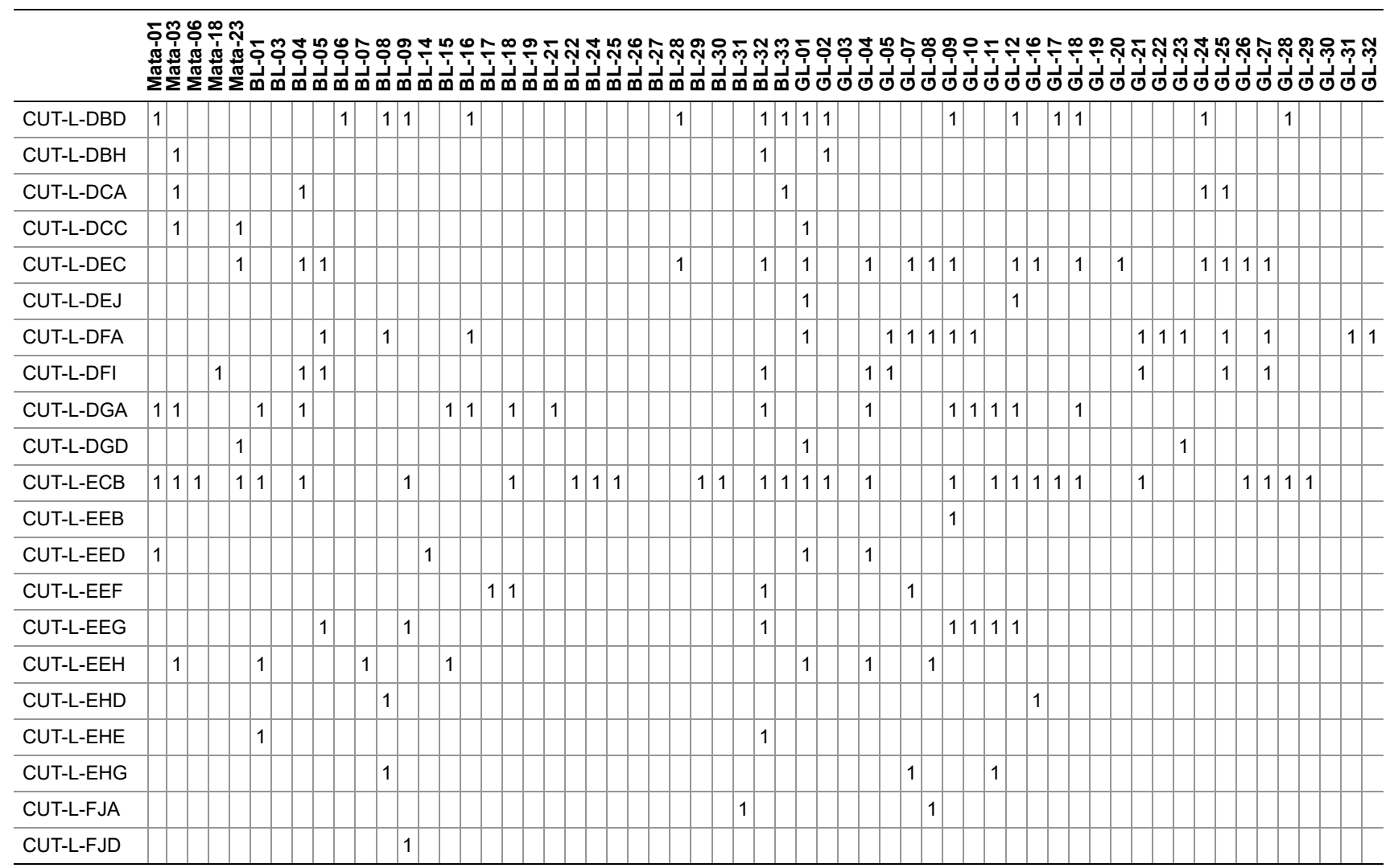

TABLE 4. Presence-absence matrix of St. Bathans Member Myrtaceae dispersed cuticle taxa.

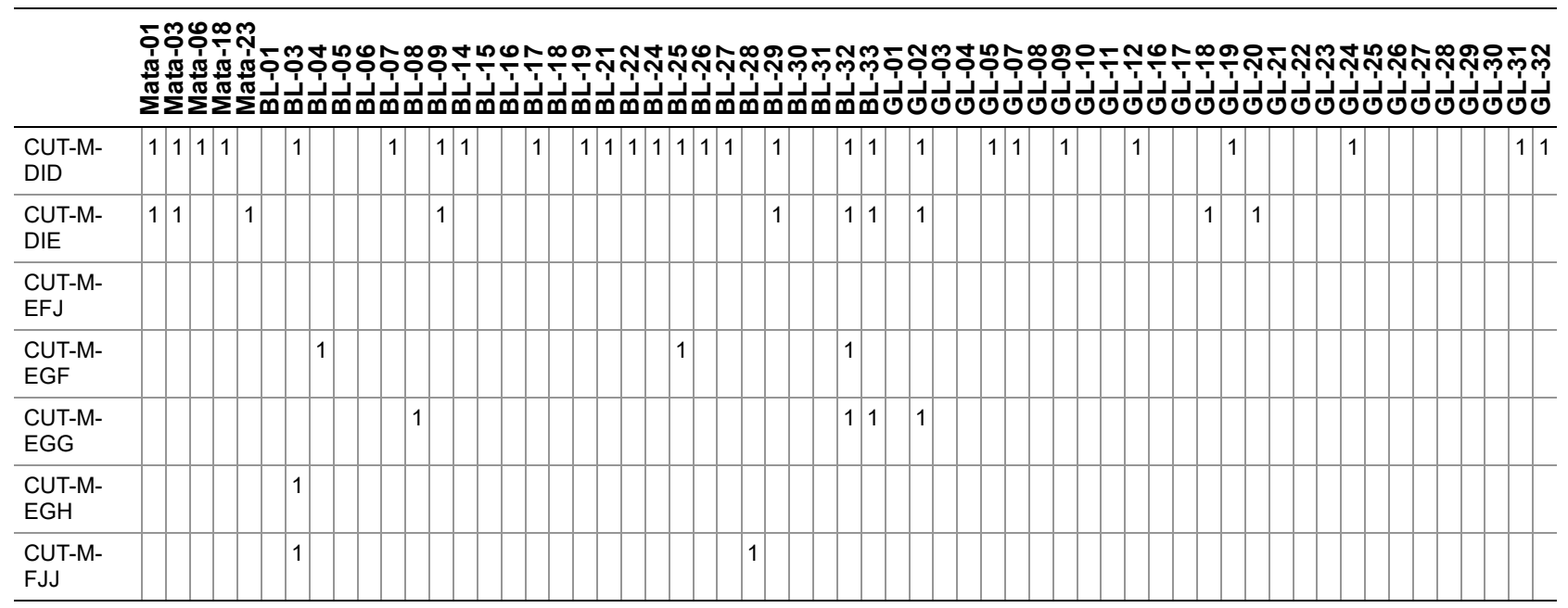

mataurarensis (Couper, 1953) Hekel (1972) are often present as well. Nothofagus was likely a prominent member of the vegetation, although it is recognised that much of the pollen could have blown from a distance. Only one form of podocarp pollen is ever common - Podocarpidites puteus (Mildenhall and Pocknall, 1989) - a species that was probably produced by Retrophyllum. Dacry- carpites and Dacrydiumites (Dacrycarpus and Dacrydium) are almost ubiquitous, but in low amounts, while Araucariaceae and Phyllocladus are rare. Some samples are notable for their lack of conifer pollen. The Proteaceae have been grouped very generally into five previously described taxa. This number will be a minimum, and detailed work will probably indicate that some 
TABLE 5. Presence-absence matrix of St. Bathans Member Proteaceae dispersed cuticle taxa.

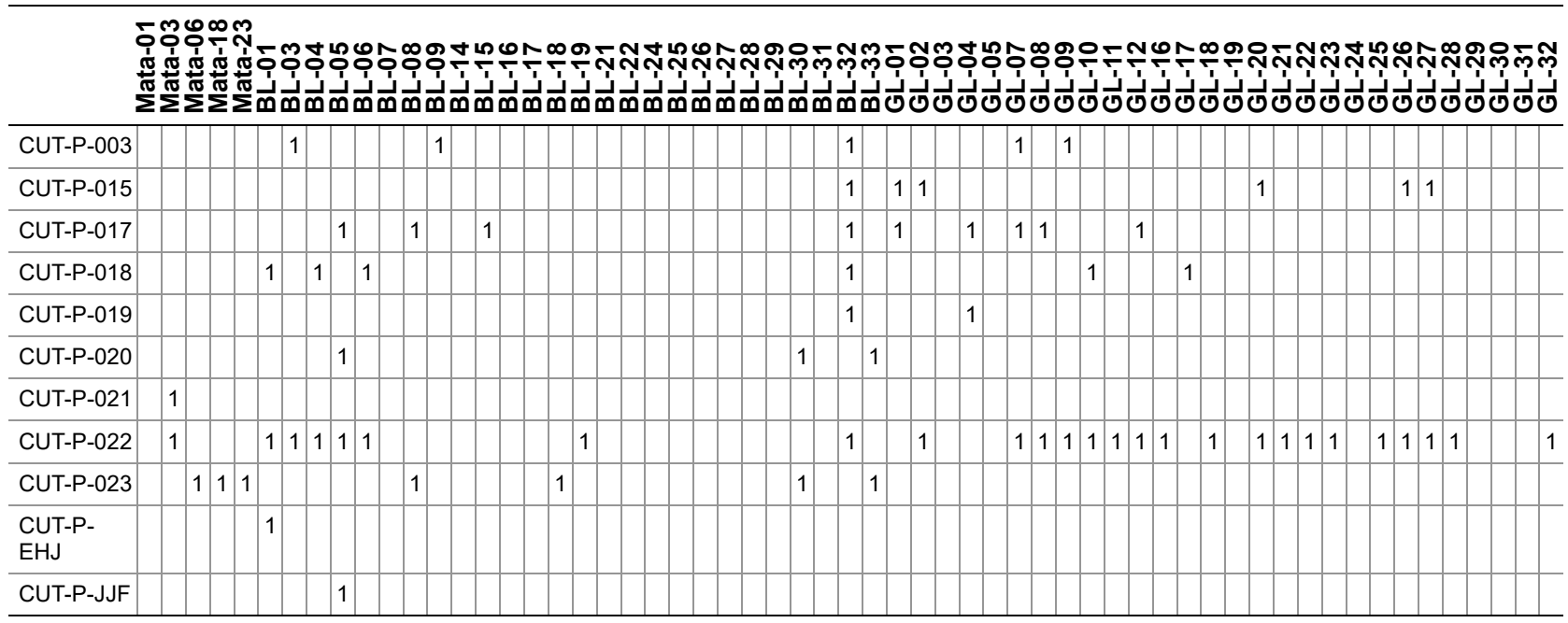

of these are new taxa. Proteaceae are typically represented by two or three of these taxa per sample. Fern or bryophyte spores and pollen of Arecaceae, Myrtaceae, Euphorbiaceae and Casuarinaceae is generally present, although often uncommon. Myrtaceae dominates over Nothofagidites in GL-05. Most spore taxa and many angiosperms only make rare appearances. Scattered occurrences of Ericaceae pollen suggest some shrubby vegetation. Pollen of putative aquatic or swamp plants such as Liliacidites, Cyperaceae, Tricolpites reticulatus (Gunnera), are present, but are always uncommon. This suggests that open swamp or marsh communities were at least restricted.

Charcoal is conspicuous by its absence and suggests fire was entirely absent. In general the palynological spectra indicates a predominance of closed, rainforest conditions to the virtual exclusion of herbfields.

\section{SYSTEMATIC PALAEONTOLOGY}

Arecaceae Berchtold and Presl, 1820 Figure 7

Specimen and occurrence. LX1777 (BL-30).

Notes. Pinnate, palm fronds, pinnae overlapping, c. $25-30 \mathrm{~mm}$ wide. Cuticle is present although no useful preparations were achieved.

\section{Proteaceae Jussieu, 1789 \\ Taxon MANU-57 (new)}

Figure 8

Reference specimen. LX1700 (GL-05).

Distinguishing features. Differs from all other Manuherikia Group taxa in its imparipinnate construction and robust teeth.
Description. Construction: imparipinnate. Terminal leaflet size: length $40 \mathrm{~mm}$, width $20 \mathrm{~mm}$, microphyll. Terminal leaflet shape ovate, apex acute, base acute. Petiole unknown. Margin toothed. First order venation pattern externodromous. Development normal. Midrib c. $1.6 \%$ width of mid-lamina. First order lateral veins probably numbering at least six to seven on each side of midrib, details indistinct. Teeth robust, sharp, c. $2 \mathrm{~mm}$ high, about four up each side of terminal leaflet, perhaps slightly more on lateral leaflets, separated by a smooth sinus.

Cuticle. CUT-P-018 of Pole (1998)

Notes. A single specimen appears to be a fragment of an imparipinnate leaf. The terminal leaflet shows the margins were robustly toothed, but the venation is unclear. The cuticle was regarded as Musgravea by Pole (1998) on the basis of the dense covering of small diameter, thickened, cylindrical trichome bases and striations over the subsidiary cells (see Carpenter et al.,1994b; Carpenter and Pole, 1995). However, the imparipinnate leaf of MANU-57 makes it distinct from the simple leaves of the extant Musgravea species.

Taxon MANU-58 (new)

Figure 9, 10

Reference specimen. LX1784 (GL-03).

Distinguishing features. Differs from all other Manuherikia Group taxa in its imparipinnate construction, or deeply, pinnately-lobed, and small teeth.

Description. Leaves up to at least $15 \mathrm{~cm}$ long, imparipinnate or deeply, pinnately-lobed with an apical lobe, and from one to three lobes on each side of a mid-rib, which extends as a petiole. The 
Pole: Early miocene St. Bathans Paleo

TABLE 6. Presence-absence matrix of St. Bathans Member 'other angiosperm' dispersed cuticle taxa.

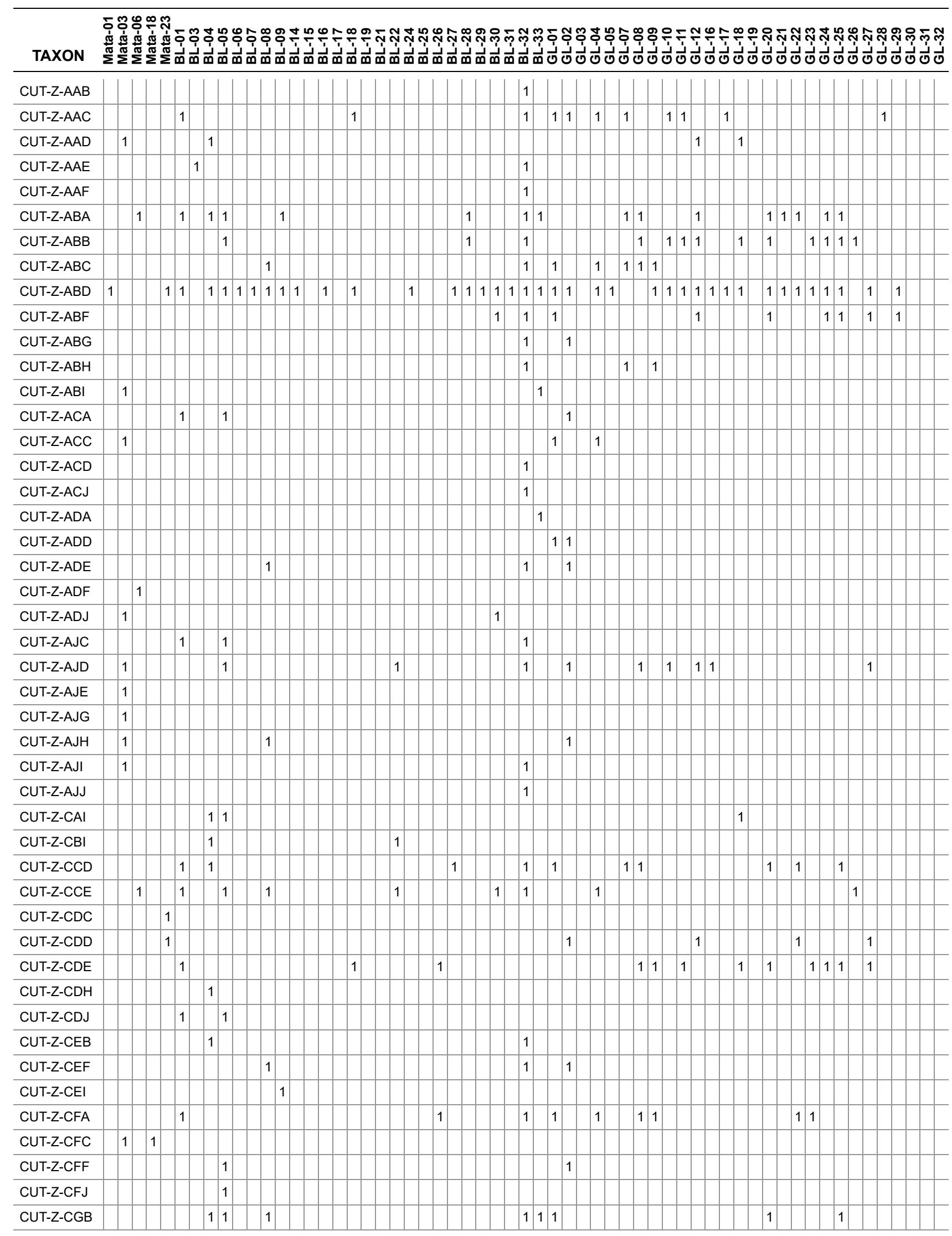


TABLE 6 (continued).

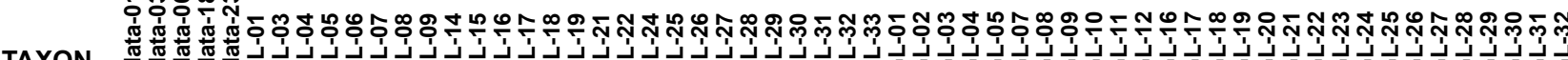

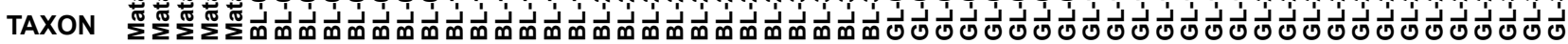

\begin{tabular}{|c|c|c|c|c|c|c|c|c|c|c|c|c|c|c|c|c|c|c|c|c|c|c|c|c|c|c|c|c|c|c|c|c|c|c|c|c|c|}
\hline CUT-Z-CGC & & & & & & & & & & & & & & & & & 1 & & & & & & & & & & & & & & & & & & & & \\
\hline CUT-Z-CGE & & & & & 1 & & & 1 & & & & & & & & & 1 & & & & & 1 & & 1 & & & & & 1 & & & & & & & & \\
\hline CUT-Z-CGG & & & & & & & 1 & & & & & & & & & 1 & & 1 & & & & & & & & & & & & & & & & & & & \\
\hline CUT-Z-CGJ & & & & & 1 & & & & & & & & & & & & & & & & & & & & & & & & & & & & & & & & \\
\hline CUT-Z-CJI & & & & & 1 & & & & & & & & & & & & & & & & & & & 1 & & & & & & & & & & & & & \\
\hline CUT-Z-DDG & 1 & & & & & & & & & & & & & & & & & 1 & & & & & & & & & & & & & & & & & & & \\
\hline CUT-Z-DDH & & & & & & & & & & & & & & & & & 1 & & 1 & & & 1 & 1 & & & & & & & 1 & & & & & & & \\
\hline CUT-Z-ECF & & & 1 & & & & & & & & & & & & & & & & & & & & & & & & & & & & & & & & & & \\
\hline CUT-Z-ECG & & & & & & & & & & & & & 1 & & & & & & & & & & & & & & & & & & & & & & & & \\
\hline CUT-Z-ECH & & & & & & & & & & & & & & & & & & & 1 & & & & & & & & & & & & & & & & & & \\
\hline CUT-Z-EDB & & & & & & & & & 1 & & & & & & & & & & 1 & & & & & & & 1 & & & & & & & & & & & \\
\hline CUT-Z-EDD & & & & & & & & & & & & & & & & & & & 1 & & & & & & & & & & & & & & & & & & \\
\hline CUT-Z-EDH & & & & & & & & 1 & & & & & & & & & & 1 & & & & & & & & & & & & & & & & & & & \\
\hline CUT-Z-EEA & & & & & & & & & & & & & & & & & 1 & & & & & & & & & & & & & & & & & & & & \\
\hline CUT-Z-EGA & & & & & & & & & 1 & & & & & & & & & & & & & 1 & & 1 & & & & & & & & & & & & & \\
\hline CUT-Z-EGB & 1 & & & & & & & & & & 1 & & & & & 1 & & & & & & & & & & & & & & & & & & & & & \\
\hline CUT-Z-EIC & & & & & & & & & & & & & 1 & 1 & & & & & & & & & & & & & & & & & & 1 & & & & & \\
\hline CUT-Z-EID & & & & & & & & & & & & & & & & & & 1 & & & & & & & & & & & & & & & & & & & \\
\hline CUT-Z-FCC & & & & & 1 & & & & & & & & & & & & & & & & & 1 & & & & & & & & & & & & 1 & & & \\
\hline CUT-Z-FAA & & 1 & & & & & & & & & & & & & & & & & & & & & & & & & & & & & & & & 1 & & & \\
\hline CUT-Z-FAE & & & & & & & & & & & & & & & & 1 & & & & & & & & & & & & & & & & & & & & & \\
\hline CUT-Z-FAJ & & & & & & & & & & & & & & & & & & & & & & 1 & & & & & & & & & & & & & & & \\
\hline CUT-Z-FBB & & & & & & & & & & & & & & & & & & & & & & & & & & & & & & & & 1 & & & & & \\
\hline CUT-Z-FBC & & & & & & & & & & & & & & & & & & & & & & & & & & & & & & & & & & & 1 & & \\
\hline CUT-Z-FBD & & & & & & & & & & & & & & & & & & & & & & & & & & & & & & & 1 & & & & & & \\
\hline CUT-Z-FBE & & & & & & & & & & & & & & & & & & & & & & & & & & & & & & & & & & 1 & & & \\
\hline CUT-Z-FBG & & & & & & & & & & & & & & 1 & & & & & & & & & & & & & & & & & & & & & & & \\
\hline CUT-Z-FBH & & & & & & & & & & & & & & & & & & & & & & & & & & & & & & & & & 1 & & & & \\
\hline CUT-Z-FBI & & & & & & & & & & & & & & & & & & & & & & & & & & & & & & & & 1 & & & & & \\
\hline CUT-Z-FCA & & & & & & & & & & & & & & & & & & & & & & & & & & & & & 1 & & & & & & & & \\
\hline CUT-Z-FCB & & & & & & & & & & & & & & & & & & & & & & & & & & & & & & & & & & & 1 & & \\
\hline CUT-Z-FCD & & & & & & & & & & & & & & & & & & & & & & & & & & & & & & & & & & 1 & 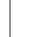 & & \\
\hline CUT-Z-FCJ & & & & & & & & & & & & & & & 1 & & & & & & & & & & & & & & & & & & & & & & \\
\hline CUT-Z-FEC & & & & & & & & & & & & & & & & & 1 & & & & & & & & & & & & & & & & & & & & \\
\hline $\begin{array}{l}\text { CUT-Z-FEE } \\
\text { is P-FEE }\end{array}$ & & & & & & & & & & & & & & & & & & 1 & & & & & & & & & & & & & & & & & & & \\
\hline CUT-Z-FFB & & & & & & & & & & & & & & & & & & & 1 & & & & & & & & & & & & & & & & & & \\
\hline CUT-Z-FFC & & & & & & & & & & & & & & & & & & & & & & & & & & & & & & & & & & 1 & & & \\
\hline CUT-Z-FFD & & & & & & & & & & & & & & & & & & & 1 & & & & & & & & & & & & & & & & & & \\
\hline CUT-Z-FFE & & & & & & & & & & & & & & & & & & & & & & 1 & & & & & & & & & & & & & & & \\
\hline CUT-Z-FFG & & & & & & & & & & & & & & & & & & & & & & & & & & 1 & & & & & & & & & & & \\
\hline CUT-Z-FGJ & & & & & & & & & & & & & & & & & & & 1 & & & & & & & & & & & & & & & & & & \\
\hline CUT-Z-FJF & & & & & & & & & & & & & & & & & & & 1 & & & & & & & & & & & & & & & & & & \\
\hline CUT-Z-FJG & & & & 1 & & & & & & & & & & & & & & & & & & & & & & & & & & & & & & & & & \\
\hline CUT-Z-FJH & & & & & & & & & & & & & & & & & & & & & & & & & 1 & & & & & & & & & & & & \\
\hline CUT-Z-FJI & & & & & & & & & & & & & & & & & & & 1 & & & & & & & & & & & & & & & & & & \\
\hline
\end{tabular}


Pole: Early miocene St. Bathans Paleo

TABLE 6 (continued).

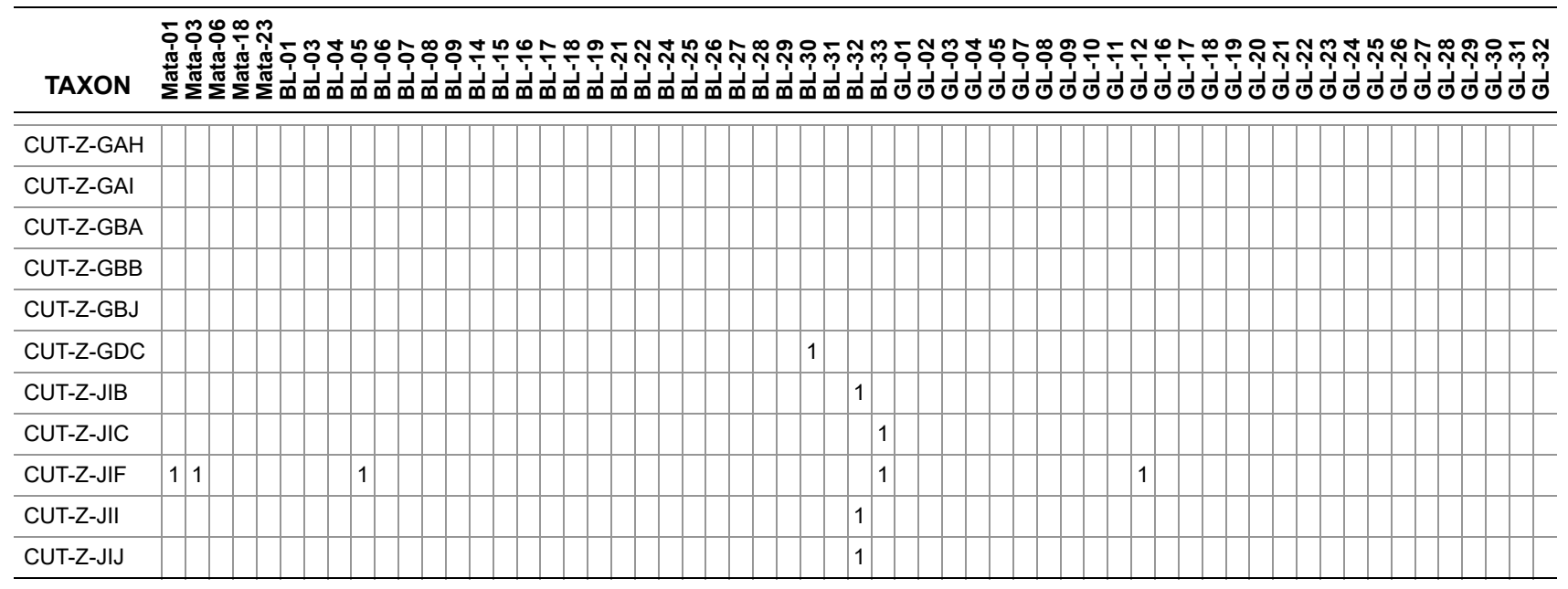

lobes are c. $20-30 \mathrm{~mm}$ wide, the widest point being about half way along the lobe. They are either curved or straight in the mid section, but curving near the midrib, so their main vein and basal margin become decurrent along the midrib. Lobe margins toothed, on both apical and basal side, the teeth small and well-spaced (c. $10 \mathrm{~mm}$ ). Lobes closely-spaced, such that the margins of adjacent lobes are more or less parallel, about a millimetre apart, or there is some overlap between adjacent lobes and of the midrib. Midrib c. $2 \%$ width of midlamina).

Cuticle. CUT-P-EHJ of Pole (2008).

Notes. The terminal leaflet/lobe resembles Euproteaciphyllum pacificum (Carpenter et al., 2012) from the Early Miocene of New Zealand, a taxon compared with tribe Macadamieae, subtribe Gevuininae. However, the later differs in the much more robust teeth and cuticle which, is strongly ridged and cells that are more distinctly buttressed. The leaf morphology recalls the Tasmanian Oligocene fossil Euproteaciphyllum polymorphum, which was said to be "unlike any living Proteaceae" (Jordan et al., 1998). E. polymorphum is about a third the size of the St. Bathans taxon, and apparently lacks a distinctly broad terminal lobe.

Nothofagaceae Kuprianova, 1962 Nothofagus Blume, 1850

Taxon MANU-6 var C, Nothofagus azureus Pole, 1993b

Figure 11

Referred specimens and occurrence. LX1934, LX1935, LX1936, LX1937 (all GL-03).

Notes. This taxon is a prominent component in some samples. Cuticle is present, but very thin, and no useful preparations have been achieved. See Pole (1993b) for placement in Nothofagus.

\section{Casuarinaceae Brown, 1814 \\ Gymnostoma Johnson, 1980 \\ Gymnostoma sp.}

Figure 12

Referred specimens and occurrence. SL3232, SL3222 (GL-30).

Notes. Gymnostoma 'articles' are identified on the basis of their distinctive rows of transversely-oriented stomatal complexes (Scriven and Hill, 1995).

$$
\begin{gathered}
\text { Cunoniaceae Brown, } 1814 \\
\text { Taxon MANU-15 } \\
\text { Figure 13, } 14
\end{gathered}
$$

Referred specimens and occurrence. SL1478 (BL-30), LX337 (GL-01), LX1972 (GL-05).

Cuticle. parataxon CUT-Z-FBE of Pole (2008).

Notes. Pole (1993c) regarded MANU-15 as Elaeocarpaceae/Cunoniaceae, citing lateral veins that tend to be directed towards the marginal sinuses, and then up the apical sides of the teeth (which are sometimes glandular). Based on subsequent work the epidermal features are also consistent with the Cunoniaceae-Elaeocarpaceae (Pole, 2008). The critical features include almost 'naked' guard cells, 'giant' stomata, strongly ribbed simple trichome bases along the veins, and 'cork warts'. A new macrofossil specimen (LX337) adds an important identification clue. This specimen is interpreted as a portion of an imparipinnate compound leaf. An apical leaflet is flanked by two lateral leaflets, and part of a further lateral leaflet (which was probably paired with another) is visible below. This construction argues against Elaeocarpaceae and especially 
TABLE 7. Presence-absence matrix of St. Bathans Member leaf parataxa.

\begin{tabular}{|c|c|c|c|c|c|c|c|c|c|}
\hline Leaf taxon & BL-32 & BL-30 & BL-15 & GL-01 & GL-02 & GL-03 & GL-04 & GL-05 & Mata-01 \\
\hline Palm fronds & & 1 & & & & & & & \\
\hline MANU-1 & & & & & & & & & 1 \\
\hline MANU-3 & & & & 1 & & & & & \\
\hline $\begin{array}{l}\text { MANU-6 var C } \\
\text { Nothofagus azureus }\end{array}$ & & 1 & & & & 1 & & & \\
\hline MANU-13 & & & & 1 & & & & & 1 \\
\hline MANU-15 & & 1 & & 1 & & & & 1 & \\
\hline MANU-17 & & & & 1 & & & & & \\
\hline MANU-33 & & & & 1 & & & & & \\
\hline MANU-35 & & & & & & & & & 1 \\
\hline MANU-46 & & 1 & & & & & & & \\
\hline MANU-49 & & & 1 & & & & & & \\
\hline MANU-50 & & & & 1 & & & & & \\
\hline MANU-51 & & & & 1 & & & & & \\
\hline MANU-52 & & & 1 & & & & & & \\
\hline MANU-53 & & & & 1 & & & & & \\
\hline MANU-54 & & & & & & & & 1 & \\
\hline MANU-55 & 1 & & & & & & & & \\
\hline MANU-56 & & & & 1 & & & & & \\
\hline MANU-57 & & & & & & & & 1 & \\
\hline MANU-58 & & & & & & 1 & & & \\
\hline
\end{tabular}

Elaeocarpus, and strongly suggests the leaves belong in the Cunoniaceae.

\section{Cunoniaceae Brown, 1814 - Elaeocarpaceae de Candolle, 1816 \\ MANU-17}

Figure 15

Referred specimens and occurrence. SL1214 (GL-01)

Notes. A single leaf base has widely-spaced and subdued teeth, with lateral veins that are probably a mixture of craspedromous and semi-craspedromous. The ultimate venation is clearly dendritic. It is considered probably conspecific with MANU-17 (Pole, 1993c). The cuticle is CUT-Z-FCJ (Pole, 2008) and is regarded as Cunoniaceae-Elaeocarpaceae.

\section{Myrtaceae Jussieu,1789 \\ Taxon MANU-53 (new)}

Figure 16

Reference specimen. LX260 (GL-01).

Distinguishing features. Differs from all other Manuherikia Group taxa in the combination of obovate shape and apparently fine venation.
Description. Size: length $75 \mathrm{~mm}$, width $34 \mathrm{~mm}$, microphyll. Shape: obovate, symmetrical, apex unknown, base unclear. Petiole unknown. Margin entire. Midrib c. $2 \%$ width of mid-lamina. First order venation pattern probably externodromous, but details unclear.

Cuticle. (?)CUT-M-EGF of Pole (2008).

Notes. A single leaf has robust cuticle, but no clear venation. It is probably obovate, and has relatively thin midrib. MANU-37 is similar, but is more elliptical. The cuticle has lid cells shows it belongs to the Myrtaceae.

\section{Sapindaceae Jussieu, 1789 Taxon MANU-46}

Figure 17

Referred specimens and occurrence. SL1481 (BL-30)

Notes. A single leaf is considered possibly conspecific with MANU-46. The specimen was almost intact, but the base broke up during mounting. The cuticle is CUT-Z-EDD, which was regarded as Sapindaceae by Pole (2008), and was compared with extant Cupaniopsis anacardioides. However, he noted that in CUT-Z-EDD the subsidiary cell flanges tend not to project over the guard cell out- 
Pole: Early Miocene St. Bathans Paleo

TABLE 8. Palynological data for St. Bathans Member samples. Percentage counts of main taxa are given for GL-3 and GL-05. For all other samples, presence is indicated by an asterix (*).

\begin{tabular}{|c|c|c|c|c|c|c|c|c|c|c|c|c|c|c|c|}
\hline Pollen Taxon & 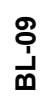 & $\frac{n}{\dot{I}}$ & $\frac{\sim}{\dot{I}}$ & $\frac{\infty}{\dot{a}}$ & $\frac{\sigma}{\bar{\Xi}}$ & $\begin{array}{l}\text { న }_{1} \\
\vec{\Delta}\end{array}$ & సَّ & 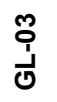 & 㸘 & 号 & 宵 & 号 & ํㅗㅇ & 움 & $\frac{5}{J}$ \\
\hline Baculatisporites & * & & & & * & & & & * & & & & & & \\
\hline Cyathidites sp. & * & & & & & * & * & * & * & & & * & * & * & \\
\hline Foveotriletes verrucosus & & * & & & & * & & * & * & & & * & * & & * \\
\hline Ischyosporites gremius & & & & & & & & & & & & & & & * \\
\hline Monolete ferns total & & & & & & & & $6 \%$ & & & & & & & \\
\hline Monolites alveolatus & & & & & & & & * & & & & * & & & \\
\hline Polypodiisporites radiatus & * & * & * & & & & & & * & * & & & & & \\
\hline Polypodiisporites sp. & * & * & * & * & * & & * & * & * & * & & * & * & & \\
\hline Polypodiaceoisporites tumulatus & & & & & & & & & & & & * & & & \\
\hline Periporopollenites polyoratus & & & & & & & & * & & & & & & & \\
\hline Trilete ferns total & & & & & & & & $1 \%$ & & & & & & & \\
\hline Verrucosisporites kopukuensis & & & & & & & & * & & & & & & & \\
\hline Araucariacites australis & & & * & & & * & & & & & & & & & \\
\hline Dacrycarpites australiensis & * & & * & & * & & * & * & * & & * & & * & & \\
\hline Dacrydiumites praecupressinoides & * & & * & & & & & & & & & & & * & \\
\hline Microalatidites paleogenicus & & * & & & & * & * & * & * & $1 \%$ & & & & & \\
\hline Microcachryidites antarcticus & & & & & & * & & & & & & & & & \\
\hline Podocarpidites sp. & & & & & & & & $1 \%$ & & $2 \%$ & & & & & \\
\hline Podocarpidites ellipticus & & & & & & & & & & * & & & & & \\
\hline Podocarpidites exiguus & & & & & & & & * & & & & & & & \\
\hline Podocarpidites puteus & * & & * & * & * & * & * & & & * & & & * & & \\
\hline Arecipites sp. & * & * & * & & * & * & * & & & $5 \%$ & & * & & * & * \\
\hline Beaupreaidites elegansiformis & & & & & & & & & * & & & & & & \\
\hline Bluffopollis sp. & & & & & & & & & * & & & & & & \\
\hline Cranwellia striata & * & * & * & & * & * & * & & * & $1 \%$ & & & & * & \\
\hline Cupanieidites reticularis & * & * & * & * & * & & * & * & & & & & & & \\
\hline Elaeocarpus/Cunoniaceae & * & & * & & * & & & & & & & & & * & \\
\hline Equisetosporites notensis & * & & & & & & & & & & & & & & \\
\hline Ericipites longisulcatus & * & & & & & & & * & * & * & & & & & \\
\hline Glencopollis ornatus & & & * & & & & & & & & & & & & \\
\hline Gothanipollis bassensis & & & * & & & & & & & & & & & & \\
\hline Gothanipollis gothani & & & * & & & & & & & * & & & & & \\
\hline Gothanipollis perplexus & & & * & & & & & & & & & * & & & \\
\hline Lateropora glabra & & & & & & & & * & & & & & & & \\
\hline Lilliacidites sp. & * & & * & & * & * & * & * & & * & & & * & * & \\
\hline Malvacearumpollis mannanensis & & & & & & * & & & & $10 \%$ & & & & & \\
\hline Malvacipollis subtilis & * & * & * & & & * & * & * & & * & & * & & & * \\
\hline Margocolporites spheripunctatus & & & * & * & & * & & & * & & & & & & \\
\hline Milfordia homeopunctata & * & & & & & & & & & & & & & & \\
\hline Monogemmites gemmatus & * & & & & & & & & & & & & & & \\
\hline Myricipites harrisii & * & * & * & & * & * & * & * & * & $1 \%$ & * & * & * & * & \\
\hline
\end{tabular}


TABLE 8 (continued).

\begin{tabular}{|c|c|c|c|c|c|c|c|c|c|c|c|c|c|c|c|}
\hline Pollen Taxon & 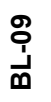 & 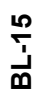 & $\frac{\sim}{\dot{J}}$ & $\stackrel{\infty}{\stackrel{\infty}{\dot{m}}}$ & 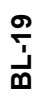 & $\begin{array}{l}\text { 옥 } \\
\overrightarrow{\text { m }}\end{array}$ & స̃ & 站 & 志 & 号 & 家 & $\begin{array}{l}\infty \\
\stackrel{1}{1} \\
\end{array}$ & 임 & 옴 & $\frac{\bar{J}}{\sigma}$ \\
\hline Myrtaceidites sp. & * & * & * & * & * & * & * & & * & $39 \%$ & & * & * & * & \\
\hline Nothofagidites asperus & * & * & * & & & * & * & $1 \%$ & * & & & & & & \\
\hline Nothofagidites cranwelliae & * & * & * & * & * & * & * & $80 \%$ & * & $26 \%$ & * & * & * & * & * \\
\hline Nothofagidites falcatus & * & & * & * & & * & * & $3 \%$ & & & & * & & & * \\
\hline Nothofagidites lachlaniae & * & * & * & & & * & * & $6 \%$ & * & $1 \%$ & & & & * & \\
\hline Nothofagidites matauraensis & * & & * & & & * & & * & & * & & * & & * & \\
\hline Nothofagidites spinosus & * & & & * & & & & & & * & & & & & \\
\hline Nyssapollenites endobalteus & & & & & & & * & & & & & & & & \\
\hline Palaeocoprosmadites zelandiae & & & & & & & & * & & & & & & & \\
\hline Parsonsidites psilatus & & & & & & & & * & * & & & & & & \\
\hline Proteacidites obscurus & & & & * & & * & & * & & * & * & & & * & \\
\hline Proteacidites pseudomoides & * & * & & & * & * & * & & * & * & & * & * & * & \\
\hline Proteacidites stratosus & & * & & & & & & & & & & & & & \\
\hline Proteacidites symphyonemoides & & & & & & & & & & & & & & * & \\
\hline Proteacidites tenuinexinus & & * & * & & & * & & & * & * & & * & & & \\
\hline Proteacidites spp. Total & & & & & & & & & & $5 \%$ & & & & & \\
\hline Rhoipites abnormis & & & & & & & & * & & & & & & & \\
\hline Rhoipites alveolatus & * & * & * & * & * & * & * & & & $\%$ & & & * & * & * \\
\hline Rhoipites aralioides & & & & & & * & & & & & & & & & \\
\hline Rhoipites titokioides & * & & & * & & & & * & & * & & & & & \\
\hline Roxburghpollis giganteus & & & & & & & & * & * & & & & & & \\
\hline Rubipollis oblatus & & * & & & & & & & * & & & & & & \\
\hline Striatricolporites pseudostriatus & & & & & & & * & & & & & & & & \\
\hline Tetracolporites ixerboides & & & & * & & & & * & & & & & & & \\
\hline Tricolpites reticulatus & * & & & & & & & & & * & & * & & & \\
\hline Triporopollenites ambiguus & & & & & & * & & & & & & & & & \\
\hline
\end{tabular}

lines and the subsidiary cells are typically elongate. These features make it distinct from $C$. anacardioides.

\section{Taxon MANU-51 (new)}

Figure 18

Reference specimen. SL650 (GL-01).

Distinguishing features. Differs from all other Manuherikia Group taxa in the combination of ovate shape, a looping zone that forms about a third or more of the lamina width and relatively wide lateral loops.

Description. Size: length c. $77 \mathrm{~mm}$, width $46 \mathrm{~mm}$, notophyll. Shape: ovate, symmetry unclear, apex unknown, base incomplete but possibly rounded. Petiole unknown. Margin entire. First order venation pattern externodromous. Development normal. Midrib c. $2 \%$ width of mid-lamina. First order lat- eral veins numbering about seven on each side of midrib, not decurrent on midrib, relatively thin, irregularly and broadly spaced (c. $10-15 \mathrm{~mm}$ ), course almost straight, or as straight-line segments, angle of divergence c. $50^{\circ}$, parallel, angle of divergence moderate to wide acute. Basal laterals not paired. Lateral loops distinct and a looping zone forming about a third or more of the lamina width. Second order and higher venation pattern very irregular, but with some tendency to admedial orientation. Marginal ultimate venation looped. Areoles poorly developed. Freely ending veinlets possibly present, but not clear.

Cuticle. Possibly CUT-Z-FJI of Pole (2008).

Notes. This is a broad leaf with relatively thin lateral veins. It is similar to MANU-4, but is regarded as having wider, less elongated lateral loops. The 


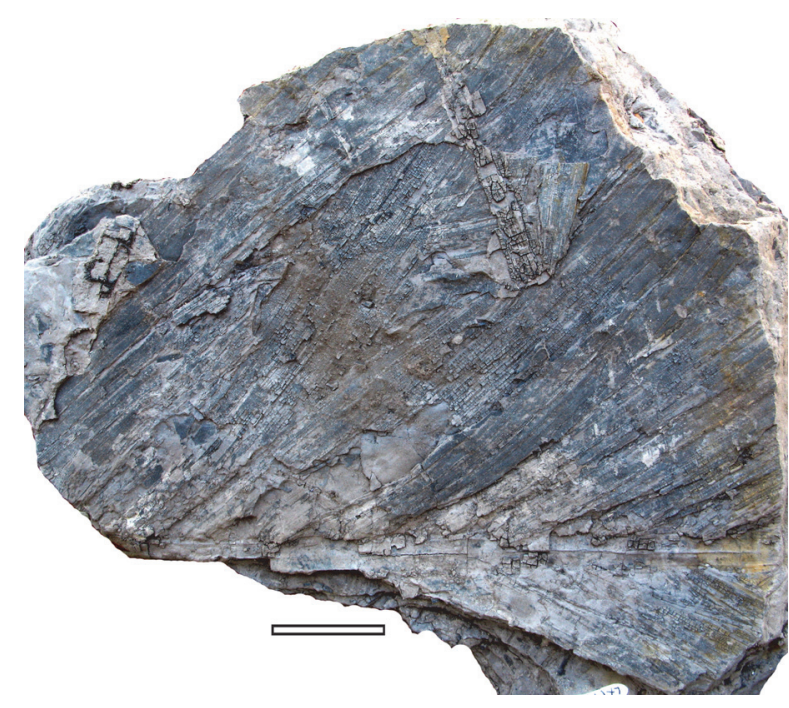

FIGURE 7. Palm frond. (LX1777, BL-30, scale bar equals $50 \mathrm{~mm}$ ).

general shape recalls MANU-12, but differs in the lateral loops, which lie well away from the margin. On that basis, it is placed in a new parataxon. The cuticle CUT-Z-FJI (Pole, 2008) is regarded as Sapindaceae, similar to extant Cupaniopsis anacardioides. The fossil leaf shows no particular resemblance to that species.

\section{Taxon MANU-61 (new)}

Figure 19

Reference specimen. SL1211 (GL-01).

Distinguishing features. Differs from all other Manuherikia Group taxa in having a distinctly asymmetrical base, cuneate on one side, decurrent on the other.

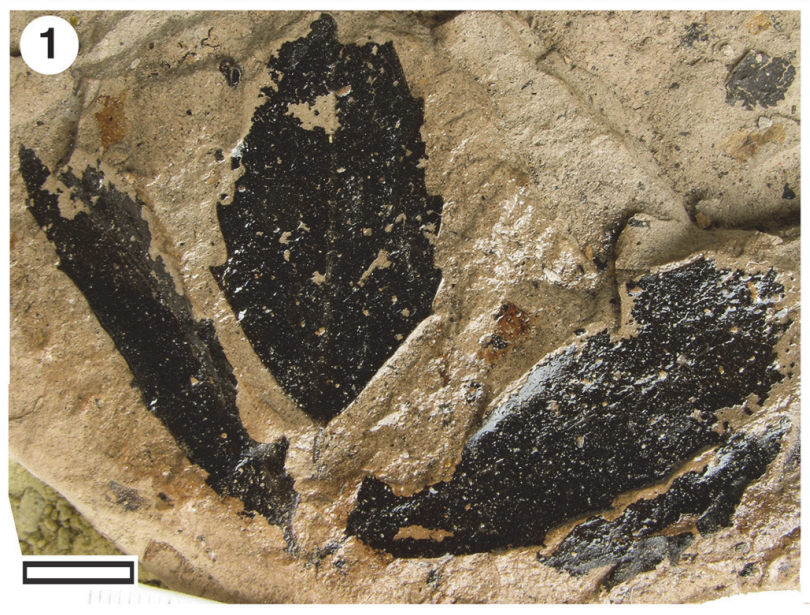

Description. Size: length unclear, probably around $60 \mathrm{~mm}$, width $30 \mathrm{~mm}$, microphyll. Shape: apex, unknown, base asymmetrical, cuneate on one side, decurrent on the other. Midrib c. $3 \%$ width of mid-lamina. Margin entire. Marginal ultimate venation looped. Venation externodromous. Slightly more than 10 lateral veins up each side of the midrib, slightly decurrent on the midvein, individual veins are thicker towards the apex. Areoles well defined with branched veinlets. Petiole is absent, but the midvein broadens abruptly at the base of the lamina, it is possibly a pulvinus.

Cuticle. CUT-Z- FJI of Pole (2008), broadly comparable with extant Cupaniopsis anacardioides.

\section{Loganiaceae R.Br. ex Martius, 1827 Strychnos Linnaeus, 1753}

Taxon MANU-50 (New), Strychnos sp. Figure 20.1

Reference specimen. SL1510 (GL-01).

Distinguishing features. Differs from all other Manuherikia Group taxa in having the basal lateral veins paired right at the lamina base, which are then decurrent into the petiole, and a lack of strongly percurrent venation.

Description. Size: length probably $60-70 \mathrm{~mm}$, width c. $40 \mathrm{~mm}$, microphyll. Shape: ovate, symmetrical, apex unknown, base concavo-convex. Petiole preserved length c. $2 \mathrm{~mm}$.

Margin entire. First order venation pattern externodromous. Development basal. Midrib c. $2 \%$ width of mid-lamina. First order lateral veins probably numbering about 4-5 on each side of midrib, basal laterals decurrent on midrib, more apical ones are not. Basal laterals paired at extreme base of lamina, entering the petiole, angle of divergence

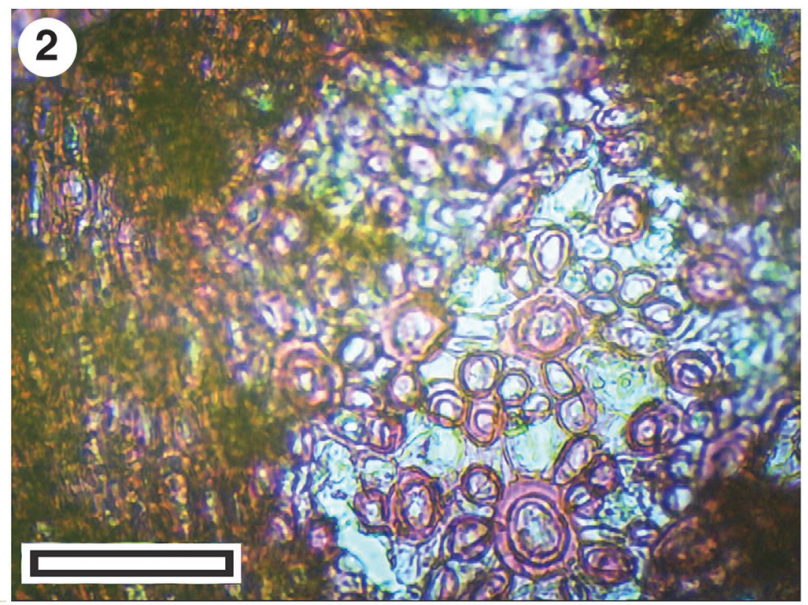

FIGURE 8. MANU-57 (GL-05). 1. In situ leaf on mudstone (LX1700, scale bar equals $10 \mathrm{~mm}$ ). 2. TLM of cuticle showing dense trichome bases or trichome attachments (scale bar equal $50 \mu \mathrm{m}$ ). 

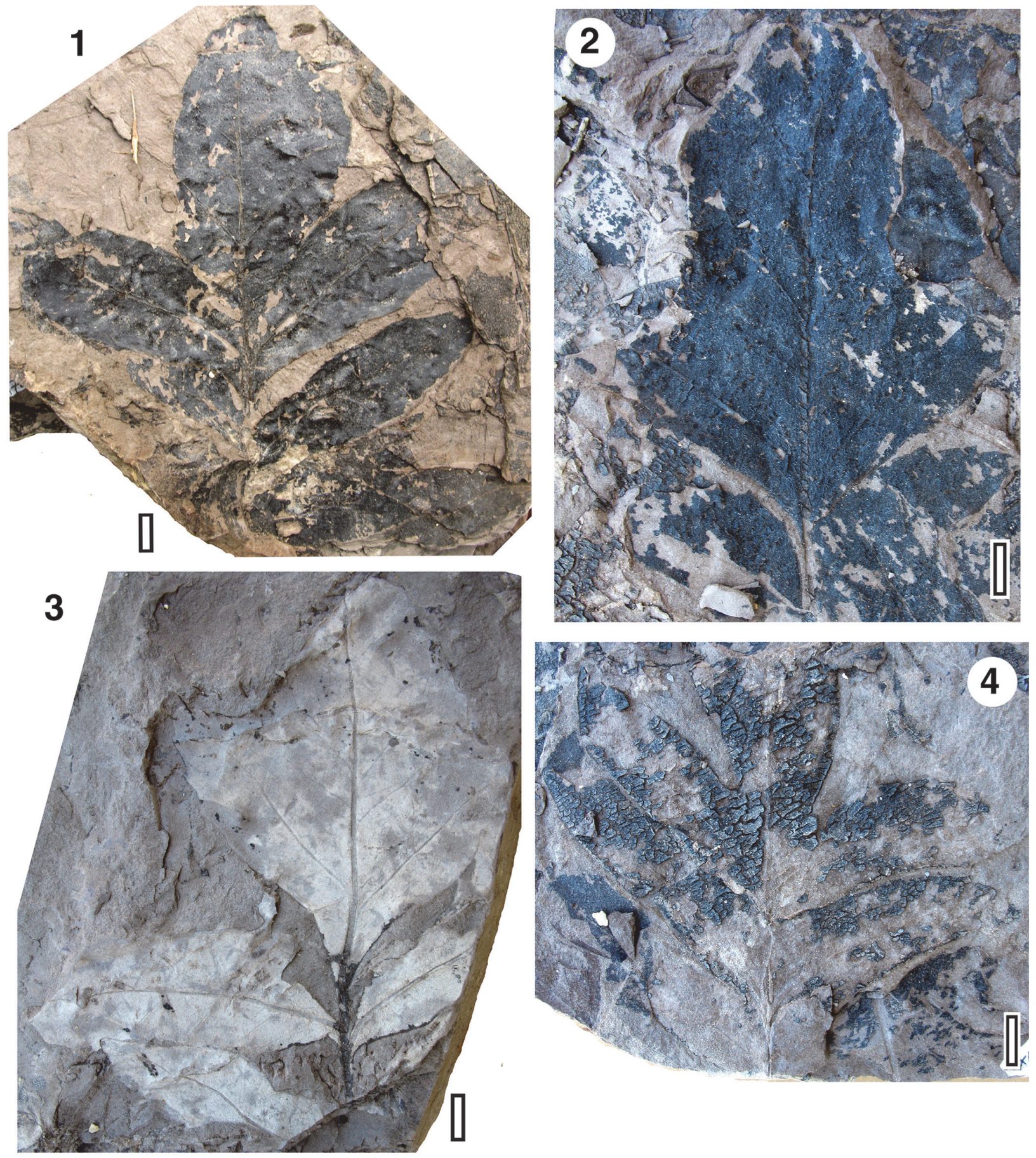

FIGURE 9. MANU-58, compound leaves in situ on mudstone (GL-03, scale bars equal 10 mm). 1. LX1784. 2. LX1786. 3. LX1399. 4. LX1785.

moderate to wide acute. Lateral loops distinct. Second order venation pattern percurrent. Third order venation irregularly reticulate, veins similar in size to the second order veins. Marginal ultimate venation probably looped. Areoles very well developed, freely ending veinlets perhaps sometimes present, unbranched.

Cuticle. CUT-Z-GFJ (new - see below)

Notes. At first glance specimen SL1510 might look like the 'tripliveined' MANU-1. However, MANU-1 is definitely Lauraceae (identified as a Cryptocarya), 

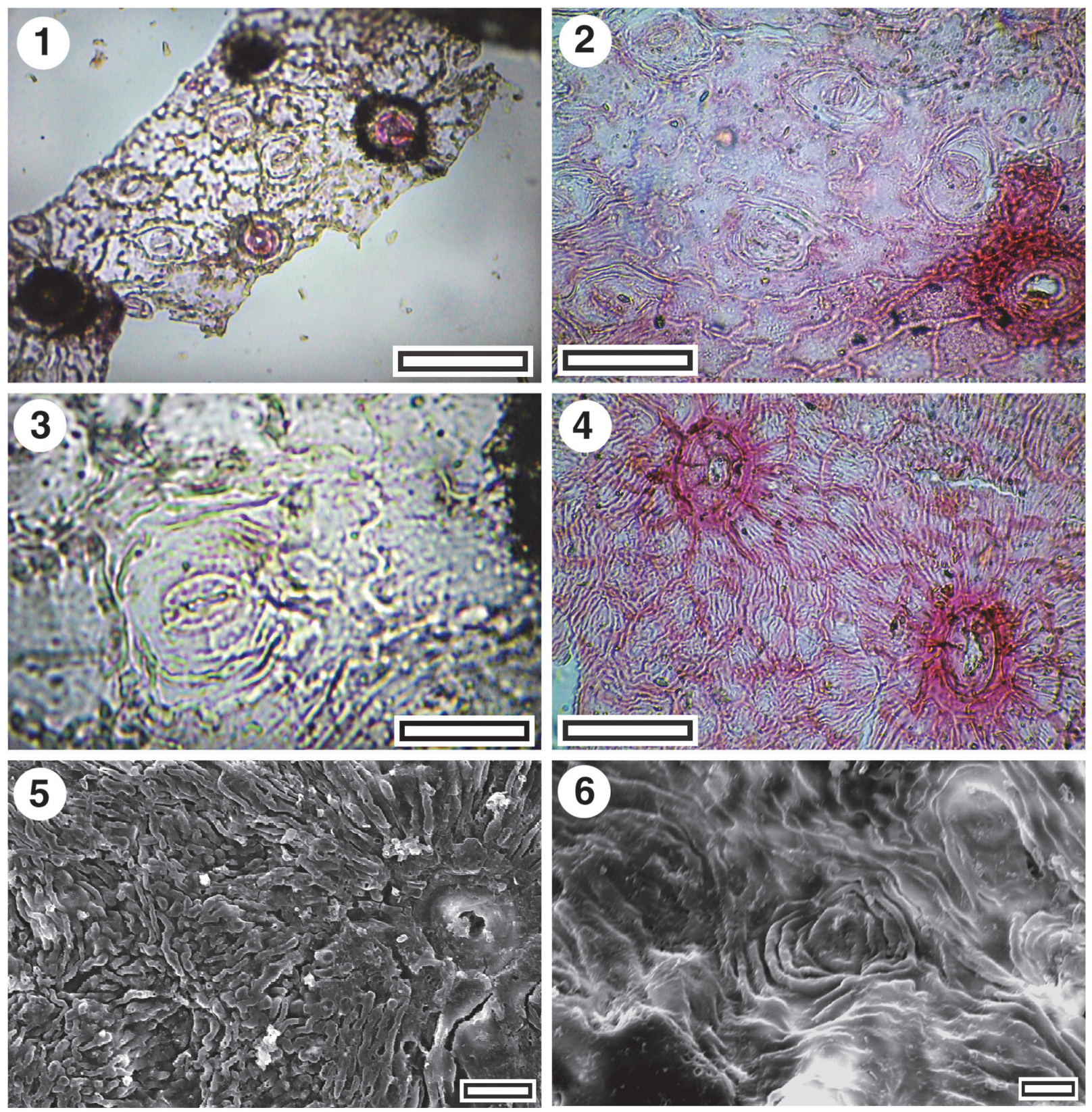

FIGURE 10. CUT-P-EHJ - cuticle of MANU-58. (all material from specimen LX1399, GL-03). 1. TLM showing several stomatal complexes and the larger, more darkly staining trichome attachment sites (scale bar equals $200 \mu \mathrm{m}$ ). 2. TLM showing several stomatal complexes and the larger, more darkly staining trichome attachment sites (scale bar $=100$ $\mu \mathrm{m})$. 3. TLM of single stomatal complex (scale bar equals $50 \mu \mathrm{m}$ ). 4. TLM of adaxial cuticle showing two multi-cellular trichome insertion bases (scale bar equals $100 \mu \mathrm{m}$ ). 5. SEM of outer cuticle surface showing radiating ridges from trichome attachment site (S-1929, scale bar equals $20 \mu \mathrm{m})$. 6. SEM of outer cuticle surface with single stomatal complex in centre (S-1906, scale bar equals $10 \mu \mathrm{m})$.

whereas the cuticle from SL1510 indicates the affinities are clearly not with Lauraceae. In SL1510 the stomatal complexes are essentially brachyparacytic with the subsidiary cells at the same level as the guard cells, but in Lauraceae the paracytic stomatal complexes have subsidiary cell that over- arch the guard cells (Hill, 1986). In MANU-1, as in other 'tripliveined' Lauraceae, the basal laterals are in detailed paired slightly above the lamina base (if not further) and the junction with the midvein is abrupt. In SL1510 the lateral veins are paired right at the lamina base, and are decurrent into the peti- 

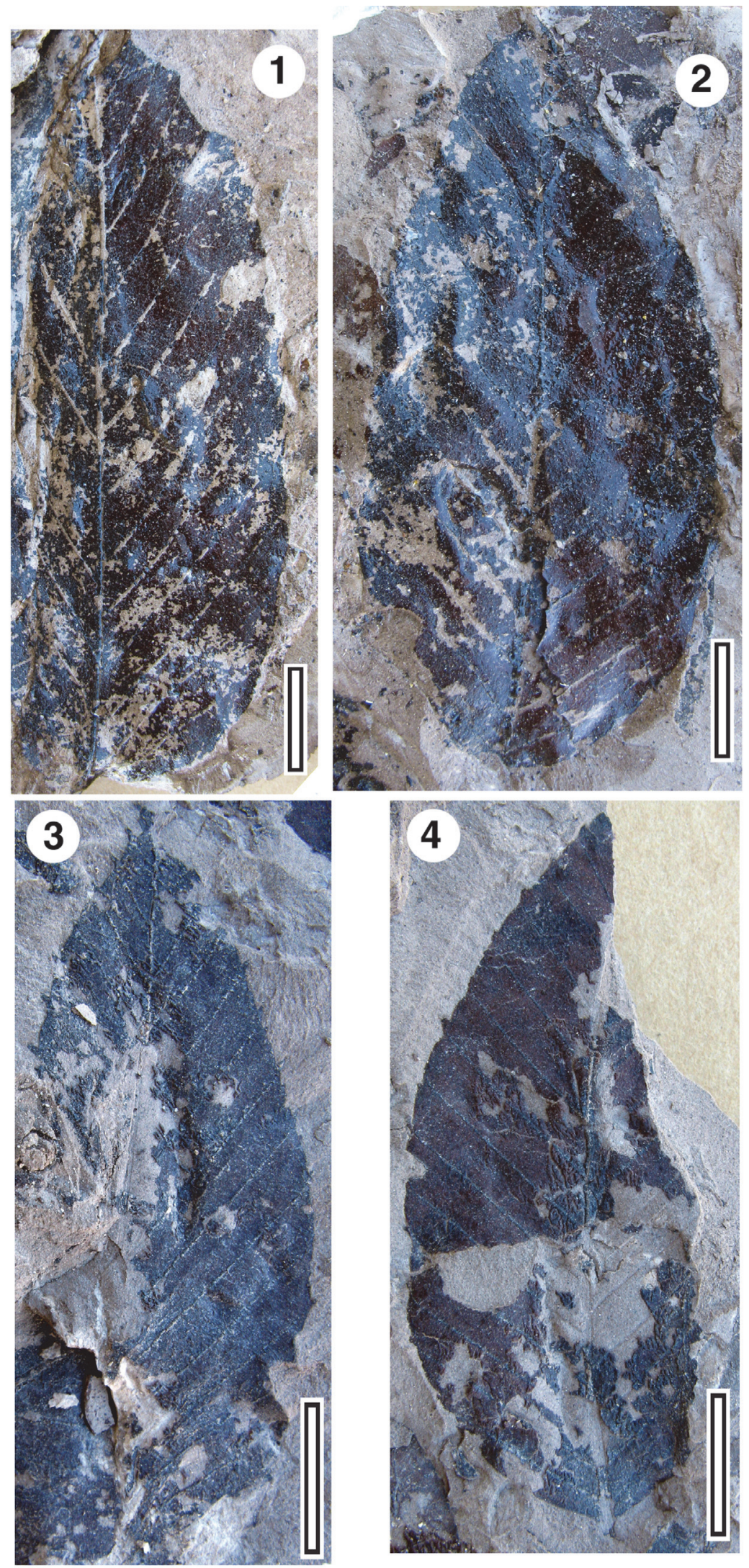

FIGURE 11. MANU-6. Nothofagus azureus. 1. LX1934. 2. LX1935. 3. LX1936. 4. LX1937 (GL-03, all scale bars equal $10 \mathrm{~mm}$ ). 

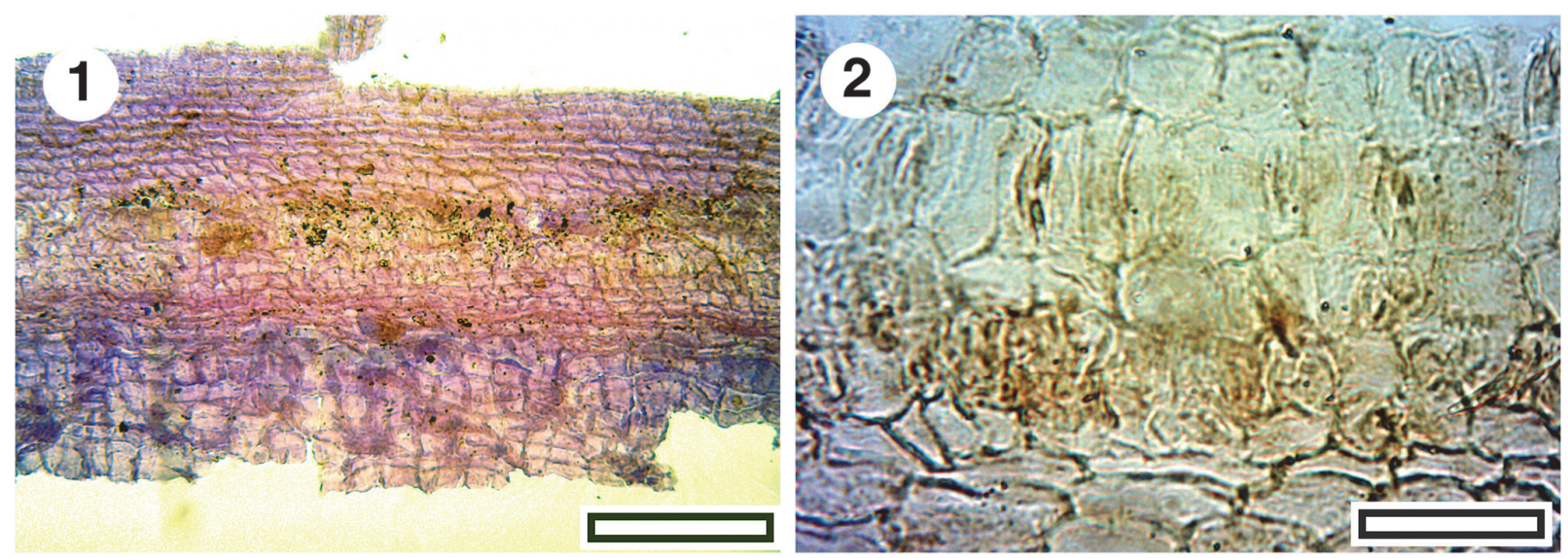

FIGURE 12. Gymnostoma sp. 1. TLM of cuticle showing a single stomatal band (SL3233, GL-30, scale bar equals 50 $\mu \mathrm{m})$. 2. TLM detail of cuticle showing four rows of transversely oriented stomata (SL3232, GL-30, scale bar equals 20 $\mu \mathrm{m})$.

ole. The specimen is distinct and is described as a new parataxon, MANU-50. It can also be distinguished from MANU-1 by the absence of strongly percurrent veins. MANU-2 has an emarginate apex, which is missing on MANU-50, but the overall shape is different.

The combination of basal lateral veins paired at the very base of the lamina, decurrent on the mid vein and entering the petiole, is very distinctive. In the context of current Australian and Malesian rainforest floras, this strongly suggests the fossil is Strychnos. The presence of a brachyparacytic stomatal complex is consistent with this. Both the fossil and extant Strychnos have subsidiary cells which are somewhat vaguely distinct and have sometimes divided in half. The genus is very large (c. 100 spp.) and no attempt is made here to compare at the species level. This is the first record of Strychnos from New Zealand. It is a component of contemporary rainforests in south-eastern Queensland/northwestern New South Wales, and its presence in the New Zealand Miocene is not surprising.

\section{Cuticle taxon CUT-Z-GFJ (New) Strychnos} Figure 20.2-20.5

Reference specimen. SL1505 (GL-01).

Description. Stomatal complexes in loose clusters, isolated, randomly oriented, essentially brachyparacytic (some subsidiary cells have divided in half), size range unimodal. Subsidiary cells elongate or isodiametric, periclinal walls thinner than normal epidermal cells, but in some cases appear to become thicker further away from the guard cells, unornamented. Guard cell pair outline clearly visible in TLM view, but not ringed by thick anticlinal walls, clearly elliptical, length $20-25 \mu$ (medium), at same level as subsidiary cells. Outer stomatal ledge unclear in TLM view, thinner than normal epidermal cells, extending over the stoma, leaving a narrow, slit-like pore.

Epidermal Cells. Epidermal cell flanges clearly visible using TLM, normal cells highly variable in shape (cells over veins not distinguished by shape), approximately the same size as the stomata, anticlinal walls wavy to sinuous, unbuttressed, smooth, unornamented.

Indumentum. Glabrous.

Notes. The cuticle of MANU-50 is unlike any previously described from the Manuherikia Group. There are broad similarities of the stomata with CUT-Z-AJC, but that differs in having stomata that tend to be concentrated in groups, and has cork warts.

\section{Unknown affinity \\ Taxon MANU-13 (Pole, 1993c)}

Figure 21

Referred specimens and occurrence. SL1212 (GL-01), SB1237 (Mata-01)

Notes. On the combination of its distinct pattern of veinlets within the areoles that are branched several times and laterals that are strongly decurrent on the midvein, these leaves are identified with MANU-13 (Pole, 1993).

Cuticle. Probably CUT-Z-CCE of Pole (2008) although the epidermal cells in the current material appear more sinuous and the trichome attachments are not as prominent. 

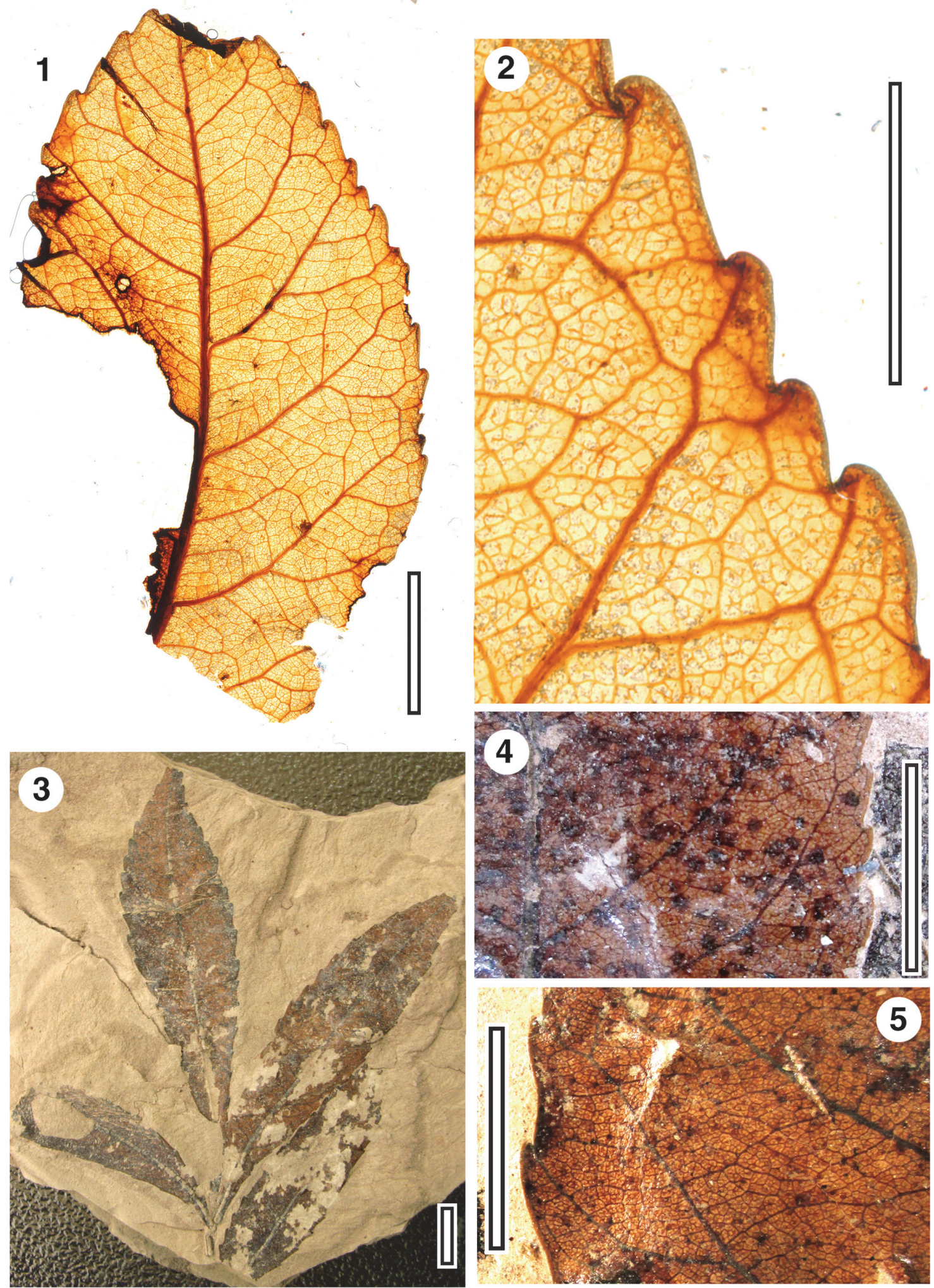

FIGURE 13. MANU-15. Elaeocarpus/Cunoniaceae leaf fossils. 1. Glycerine jelly/plastic sheet mount (SL1478, BL-30, scale bar equals $10 \mathrm{~mm}$ ). 2. Glycerine jelly/plastic sheet mount, detail of margin (SL1478, BL-30, scale bar equals 5 $\mathrm{mm})$. 3. Apparent compound leaf on mudstone matrix, (LX337, GL-01, scale bar equals $10 \mathrm{~mm})$. 4. Leaf on mudstone matrix (LX1410, GL-05, scale bar equals $5 \mathrm{~mm}$ ). 5. PVB resin mount (LX1388, scale bar equals $5 \mathrm{~mm}$ ). 

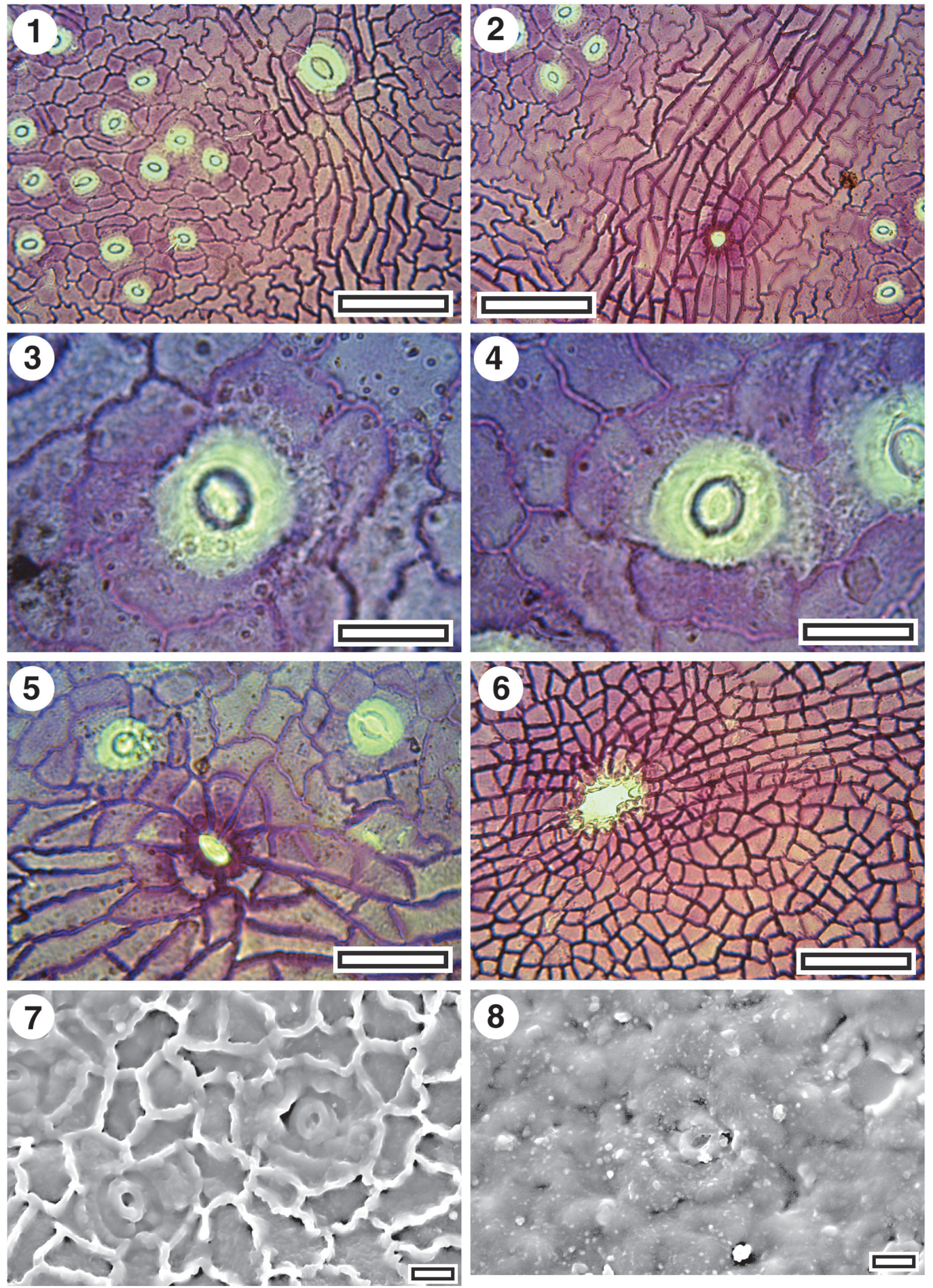

FIGURE 14. Cuticle from MANU-15 - CUT-Z-FBE (BL-30). 1. TLM with a group of normal stomatal complexes at left, and a 'giant' stomatal complexes over a vein at upper right (SL1478, scale bar equals $100 \mu \mathrm{m}$ ). 2. TLM with two groups of normal stomatal complexes separated by a vein with a simple trichome attachment (SL1478, scale bar equals $100 \mu \mathrm{m})$. 3. TLM of single stomatal complex (SL1478, scale bar equals $20 \mu \mathrm{m})$. 4. TLM of single stomatal complex (SL1478, scale bar equals $20 \mu \mathrm{m}$ ). 5. TLM of two stomatal complexes with a trichome attachment (SL1478, scale bar equals $50 \mu \mathrm{m}$ ). 6 . TLM of upper cuticle with a small 'cork wart' (SL1478, scale bar equals $100 \mu \mathrm{m}$ ). 7. SEM of inner cuticle with two stomatal complexes (S-1948, scale bar equals $10 \mu \mathrm{m})$. 8. SEM of outer cuticle with one stomatal complex in the centre (S-1948, scale bar equals $10 \mu \mathrm{m})$. 

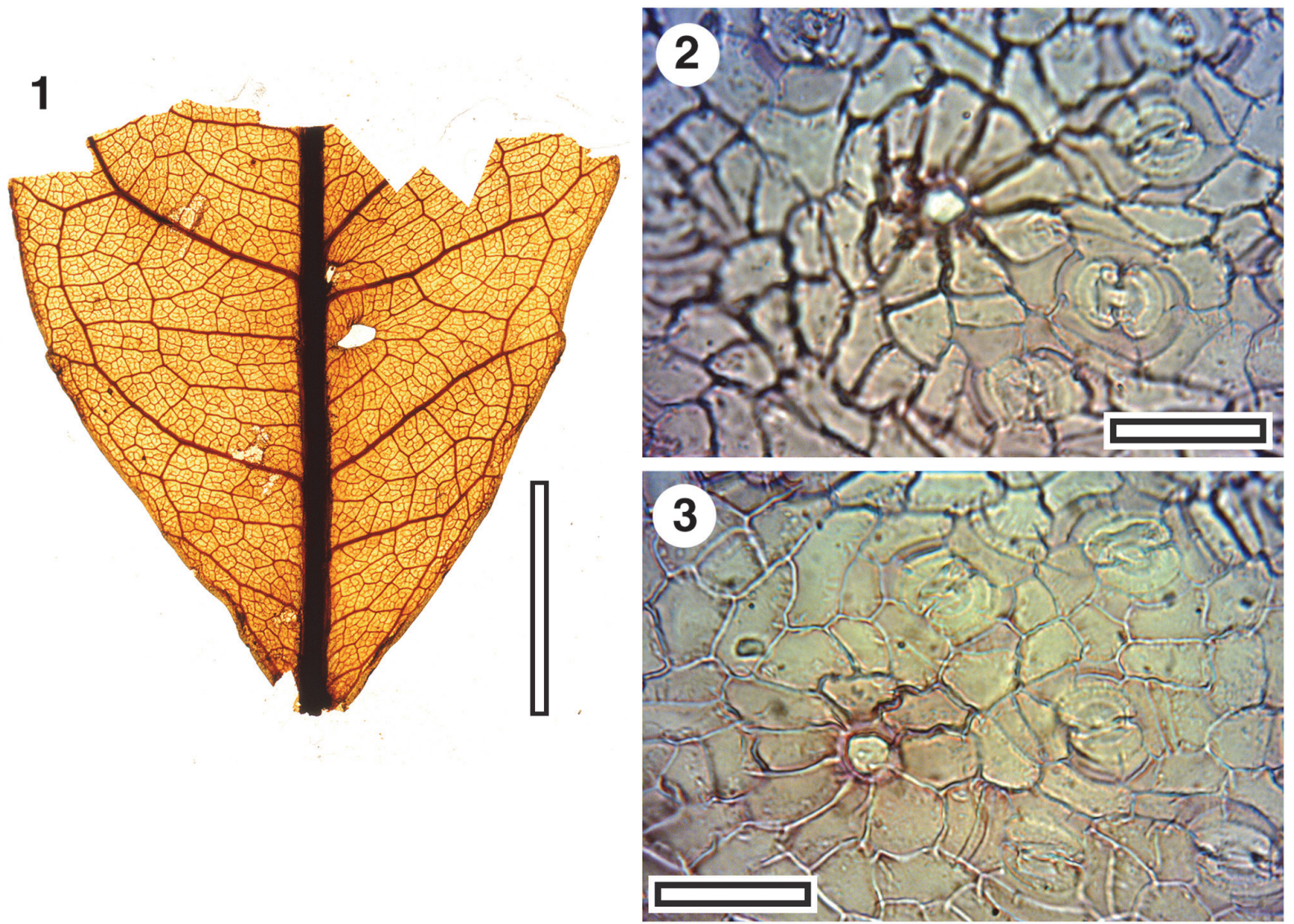

FIGURE 15. MANU-17 and CUT-Z-FCJ (SL1214, GL-01). 1. Base of leaf, glycerine jelly/plastic sheet mount (scale bar equals $10 \mathrm{~mm}$ ). 2. TLM of cuticle with stomatal complexes and single trichome attachment (scale bar equals 50 $\mu \mathrm{m})$. 3. TLM of cuticle with stomatal complexes and single trichome attachment (scale bar equals $50 \mu \mathrm{m}$ ).

Taxon MANU-49 (New)

Figure 22, 23

Reference specimen. OU29799 (BL-15)

Referred specimens and occurrence. OU29800, OU30222, OU29798.

Distinguishing features. Differs from all other Manuherikia Group taxa in having prominent and inclined teeth, in combination with relatively thin first order venation.

Description. Size: length probably c. $60 \mathrm{~mm}$, width 27-30 mm, microphyll. Shape: probably elliptical, symmetrical although the base is possibly asymmetrical, apex acuminate, base convex. Petiole unclear. First order venation pattern mixed craspedodromous and semicraspedodromous, sometimes first-order laterals appear almost bifurcating. Development normal. Midrib c. 1.0-1.4\% width of mid-lamina. First order lateral veins numbering about 6-8 on either side of midrib in the main, expanded part of the lamina, sometimes slightly decurrent on midrib, relatively thin, irregularly spaced (c. every 8-15 mm), course irregularly curved or as a series of straight segments, angle of divergence c. $50-70^{\circ}$, roughly parallel, angle of divergence moderate to wide acute. Basal laterals not paired. Lateral loops distinct. Second order venation pattern: within the lateral zones there is no clear pattern, but sometimes a relatively thick vein 'halves' the zone either vertically or horizontally by a second-order lateral, and sometimes the two vein types may 'quarter' it. Venation within the larger external loops often shows a tendency to an admedial orientation - with a relatively large vein extending from the outer limit of the loop towards the mid rib. Marginal ultimate venation branched. Areoles irregular, poorly developed, freely-ending veinlets sometimes present, unbranched.

Margin toothed (indented c. 3\% of distance to midrib in widest portion of the lamina), tooth length c. $0.4-0.7 \mathrm{~mm}$, dentate axes inclined to tangent to axis, basal side of tooth convex, apical side of tooth convex, sinuses rounded and very broad, 

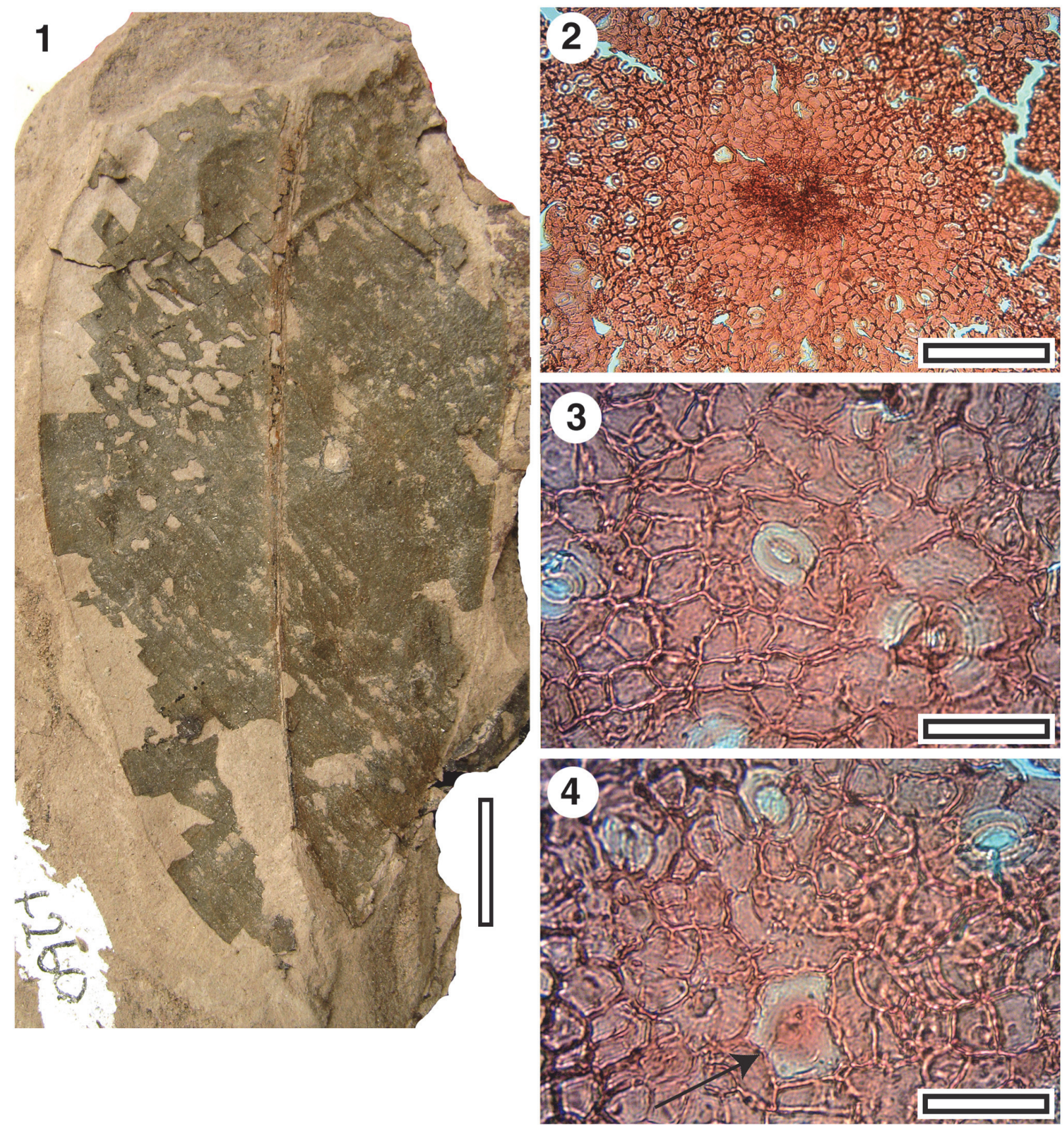

FIGURE 16. MANU-53 and (?) CUT-M-EGF (LX260, GL-01). 1. Whole leaf, in situ on mudstone (scale bar equals 10 $\mathrm{mm}$ ). 2. TLM of cuticle with numerous stomatal complexes surrounding glandular structure in centre (scale bar equals $200 \mu \mathrm{m}$ ). 3. TLM of cuticle (scale bar equals $50 \mu \mathrm{m}$ ). 4. TLM of cuticle with stomatal complexes at top and paired lid cells below, arrowed (scale bar equals $50 \mu \mathrm{m}$ ).

spacing mostly regular, mostly $3-5 \mathrm{~mm}$ apart, number of teeth per first order lateral mostly $1-2$, series simple, present along complete margin. Teeth primarily vascularised by second or third order externals, running centrally, or closer to the basal (outer) margin, which often reflexing strongly towards the tooth apex. A marginal vein, of almost equal thickness, proceeds just inside the proximal flank of the tooth to the apex. Occasionally a much finer vein is present just inside the distal tooth flank.

Cuticle. CUT-Z-ADF of Pole (2008). 

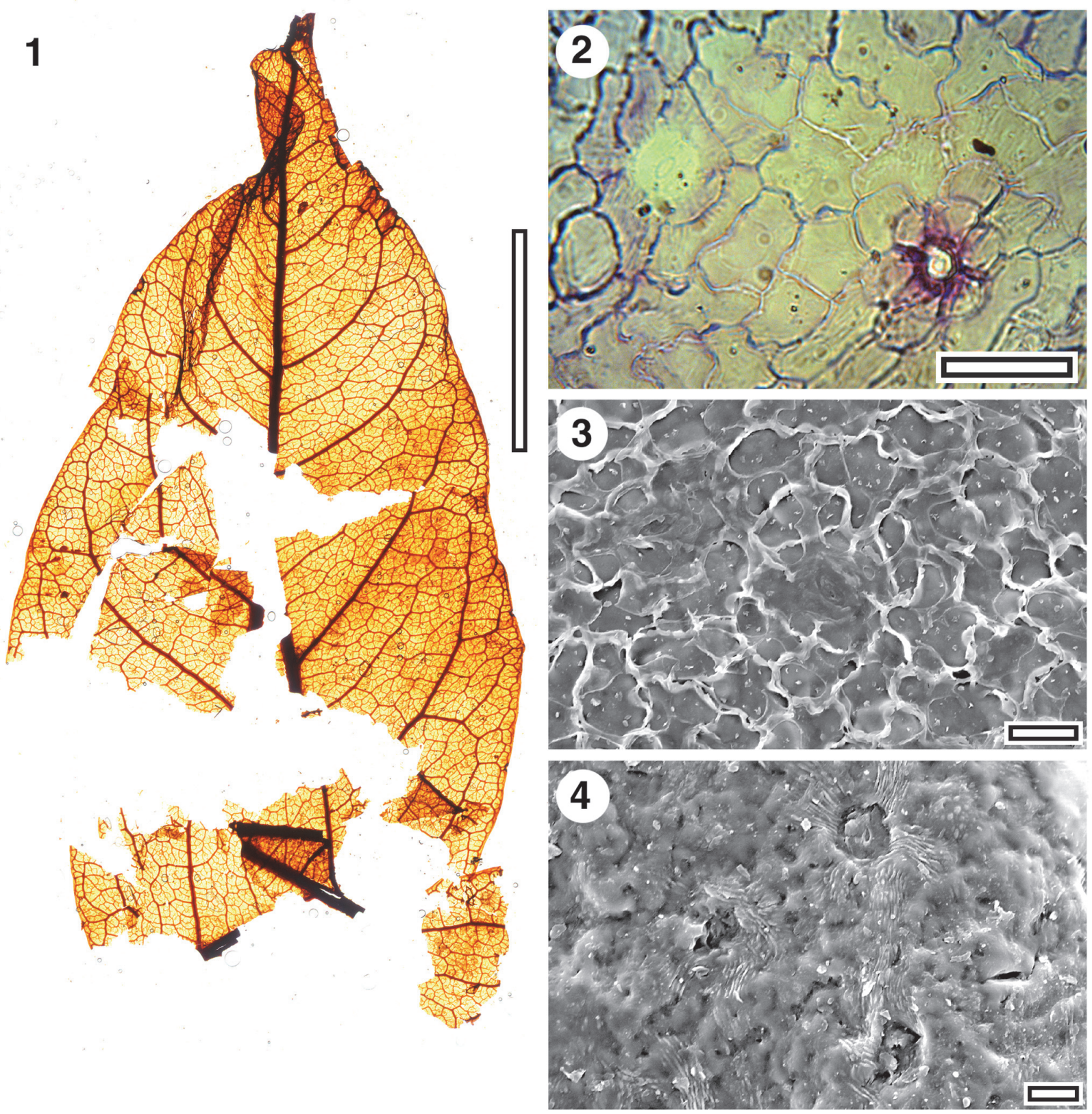

FIGURE 17. MANU-46 and CUT-Z-EDD (SL1481, BL-30). 1. A whole leaf (base was intact, but damaged during mounting), glycerine jelly/plastic sheet mount. 2. TLM of cuticle with stomatal complex to left and trichome attachment to right (scale bar equals $50 \mu \mathrm{m})$. 3. SEM of inner cuticle surface(S-1949, scale bar equals $20 \mu \mathrm{m})$. 4. SEM of outer cuticle surface with stomatal complexes surrounded by short radiating ridges (S-1949, scale bar equals $20 \mu \mathrm{m}$ ).

Notes. Several leaf specimens have broadly similar features to MANU-15, but differ in having thinner lateral venation, and more widely-spaced teeth with smoother sinuses between.

\section{Taxon MANU-52 (new)}

Figure 24

Reference specimen. OU29848 (BL-15).
Distinguishing features. Differs from all other Manuherikia Group taxa in having a (probably) elliptical lamina, with margins in mid-lamina being almost straight, and broadly spaced first order lateral veins.

Description. Size: length unclear but probably around $115 \mathrm{~mm}$, width $32 \mathrm{~mm}$, notophyll. Shape: probably elliptical with margins in mid-lamina almost straight, symmetrical, apex unknown, base 

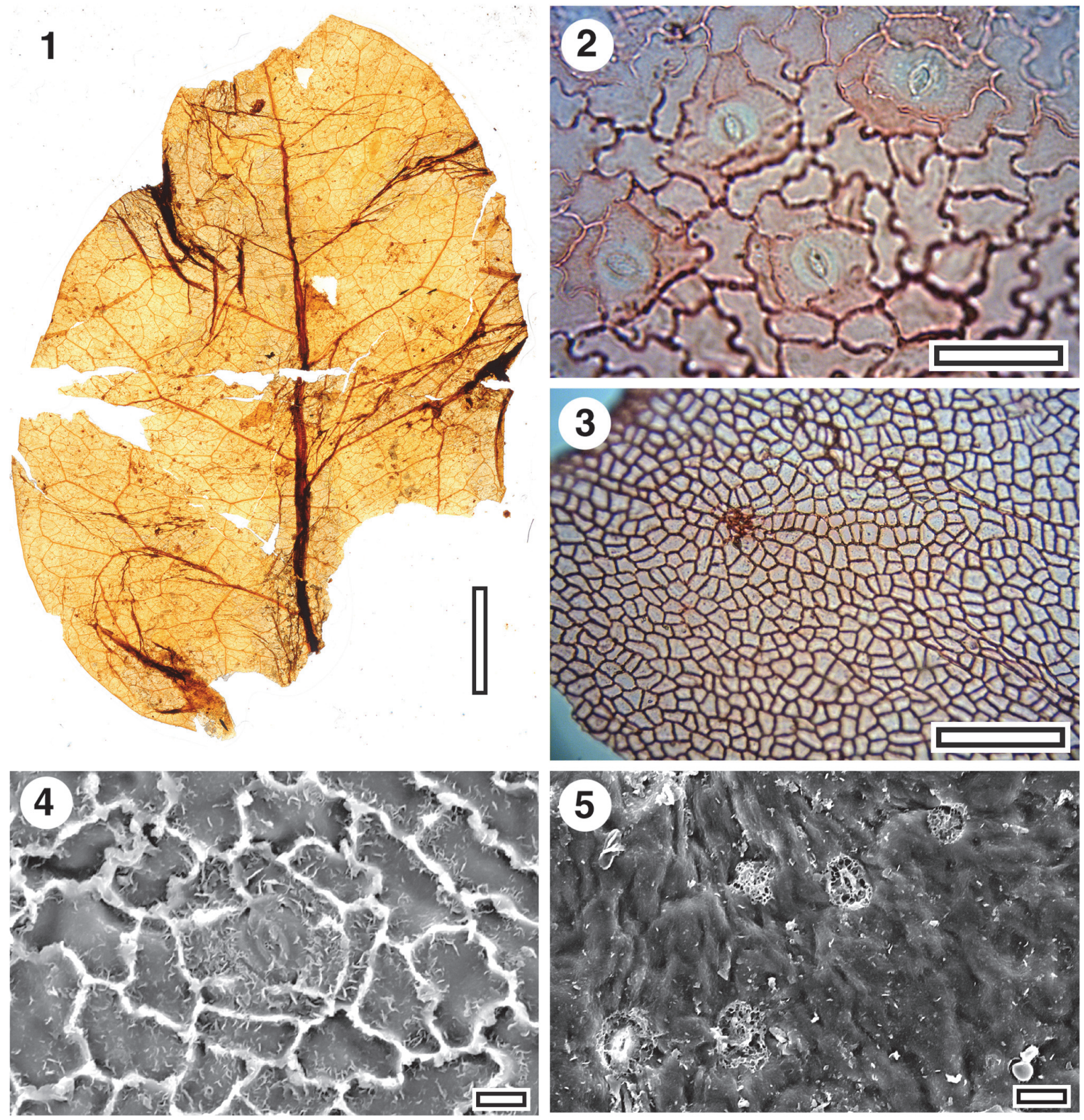

FIGURE 18. MANU-51 and (?) CUT-Z-FJI (SL6501, GL-01). 1. Whole leaf, glycerine jelly/plastic sheet mount (scale bar equals $10 \mathrm{~mm}$ ). 2. TLM of cuticle (SL6501, scale bar equals $50 \mu \mathrm{m}$ ). 3. TLM of cuticle (SL6501, scale bar equals $200 \mu \mathrm{m})$. 4. SEM of inner cuticle surface(S-1951, scale bar equals $10 \mu \mathrm{m})$. 5. SEM of outer cuticle surface(S-1951, scale bar equals $20 \mu \mathrm{m}$ ).

cuneate or decurrent. Petiole unknown. Margin entire. First order venation pattern externodromous. Development normal. Midrib c. $3 \%$ width of mid-lamina. First order lateral veins probably numbering about five on each side of midrib, not decurrent on midrib, relatively thick, irregularly and broadly spaced (c. every 15-25 mm), course curved, angle of divergence c. $50^{\circ}$. Basal laterals not paired, roughly parallel then starting to converge, angle of divergence moderate to wide acute. Lateral loops distinct. Second order venation pattern percurrent. Third order venation pattern regularly reticulate. Marginal ultimate venation fimbrial. Areoles regularly developed, freely ending veinlets sometimes present, branching once.

Cuticle. CUT-Z-AJI of Pole (2008). 

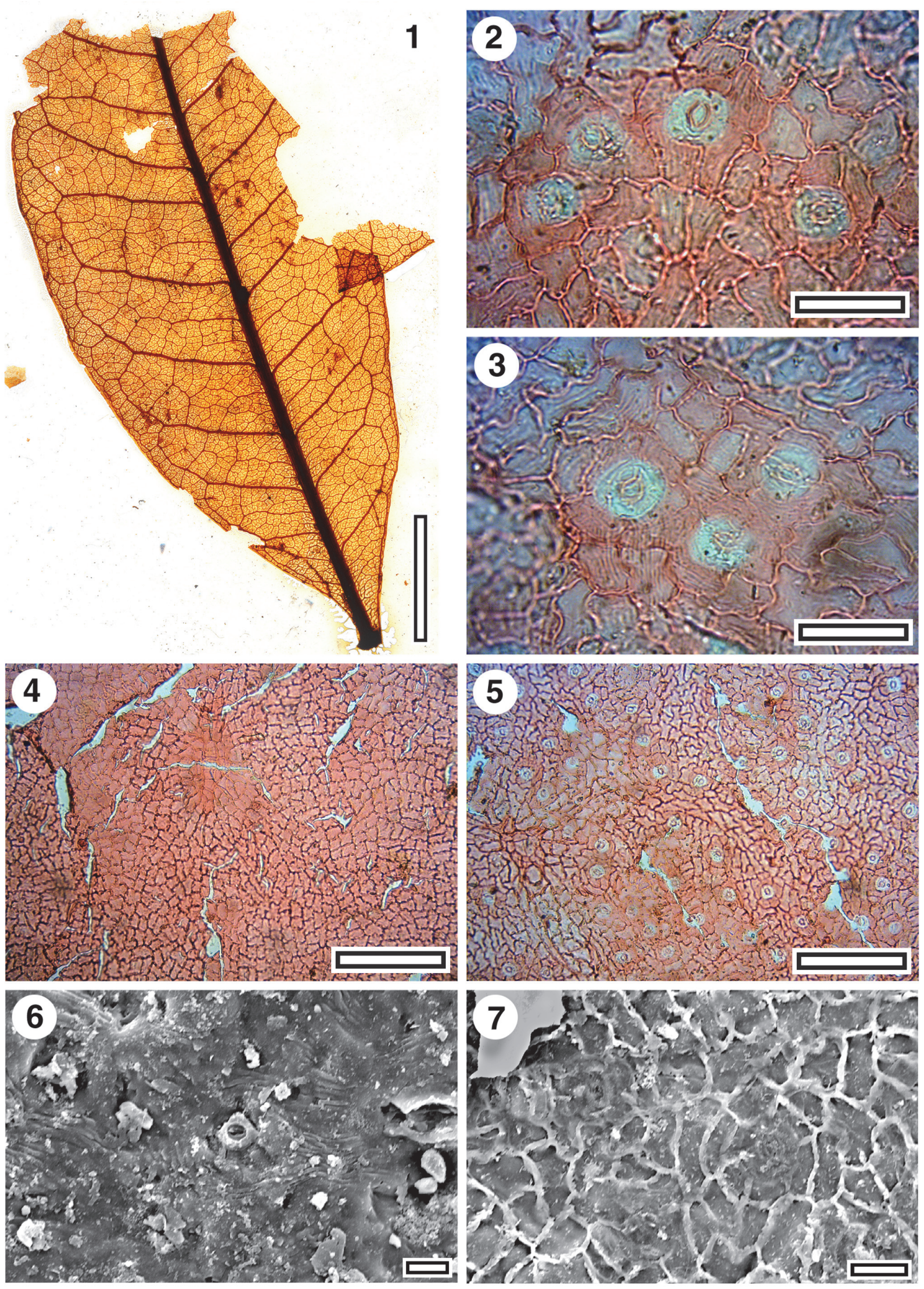

FIGURE 19. MANU-61 and CUT-Z-FJI (SL1211, GL-01). 1. Whole leaf, glycerine jelly/plastic sheet mount (scale bar equals $10 \mathrm{~mm}$ ). 2. TLM of cuticle (scale bar equals $50 \mu \mathrm{m}$ ). 3. TLM of cuticle (scale bar equals $50 \mu \mathrm{m}$ ). 4. TLM of cuticle (scale bar equals $200 \mu \mathrm{m}$ ). 5. TLM of cuticle (scale bar equals $200 \mu \mathrm{m}$ ). 6. SEM of outer cuticle surface (S-1953, scale bar equals $10 \mu \mathrm{m})$. 7. SEM of inner cuticle surface(S-1953, scale bar equals $20 \mu \mathrm{m})$. 

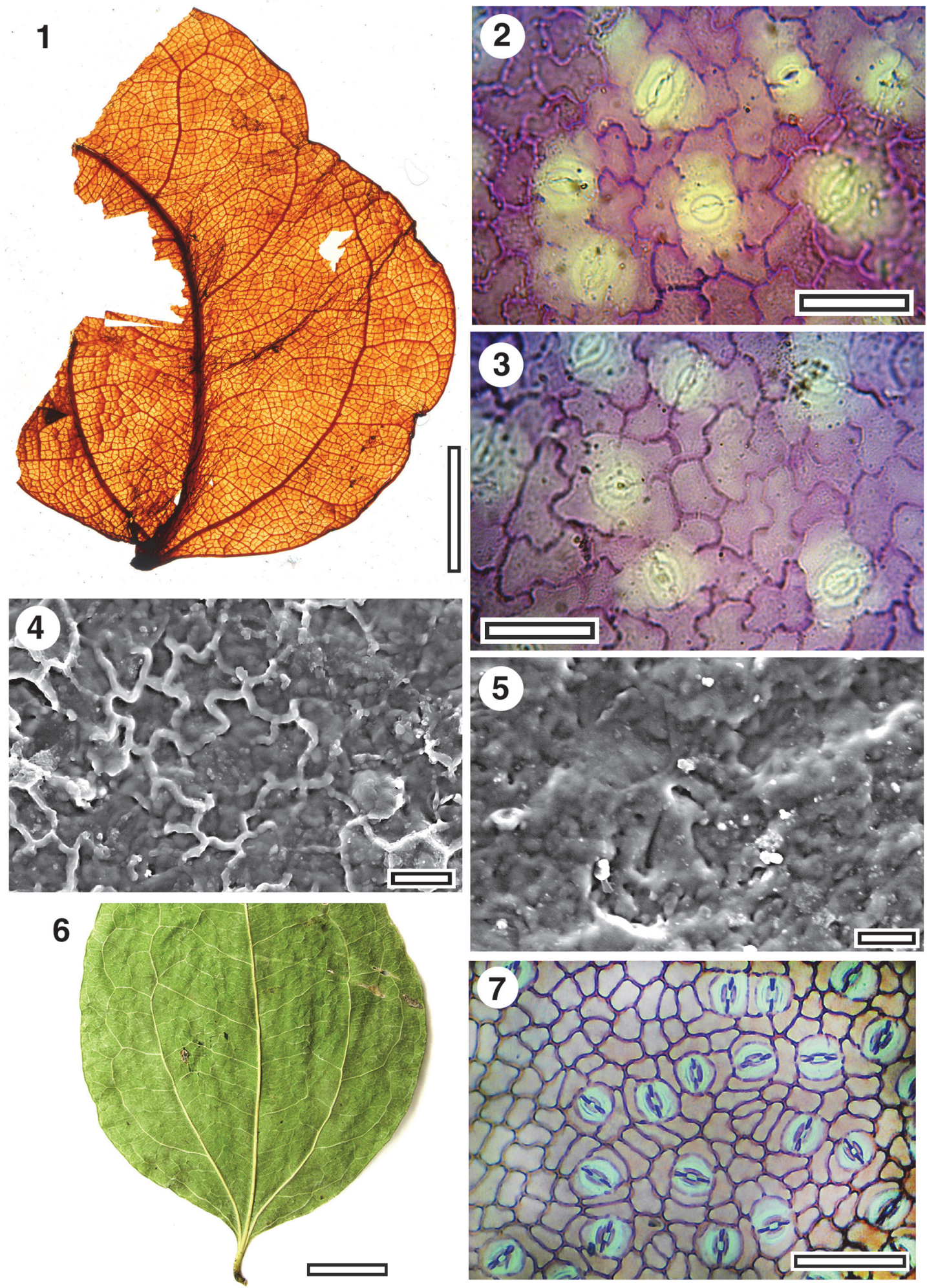

FIGURE 20. MANU-50 and CUT-Z-GFJ, Strychnos sp. (SL1510, GL-01). 1. Whole leaf, glycerine jelly/plastic sheet mount (scale bar equals $10 \mathrm{~mm}$ ). 2. CUT-Z-GFJ, TLM (scale bar equals $50 \mu \mathrm{m}$ ). 3. CUT-Z-GFJ, TLM (scale bar equals $50 \mu \mathrm{m}$ ). 4. CUT-Z-GFJ, SEM of inner cuticle surface with a single, indistinctly outlined stomatal complex (S1946, scale bar equals $20 \mu \mathrm{m})$. 5. CUT-Z-GFJ, SEM of outer cuticle surface with at least one almost cryptic stomatal complex (S-1946, scale bar equals $20 \mu \mathrm{m})$. 6. Leaf base of extant Strychnos lucida Brown (1810) (AQ837571, scale bar equals $10 \mathrm{~mm}$ ). 7. Extant Strychnos minor Dennstedt (1818) cuticle, TLM (AQ481523, scale bar equals $200 \mu \mathrm{m})$. 

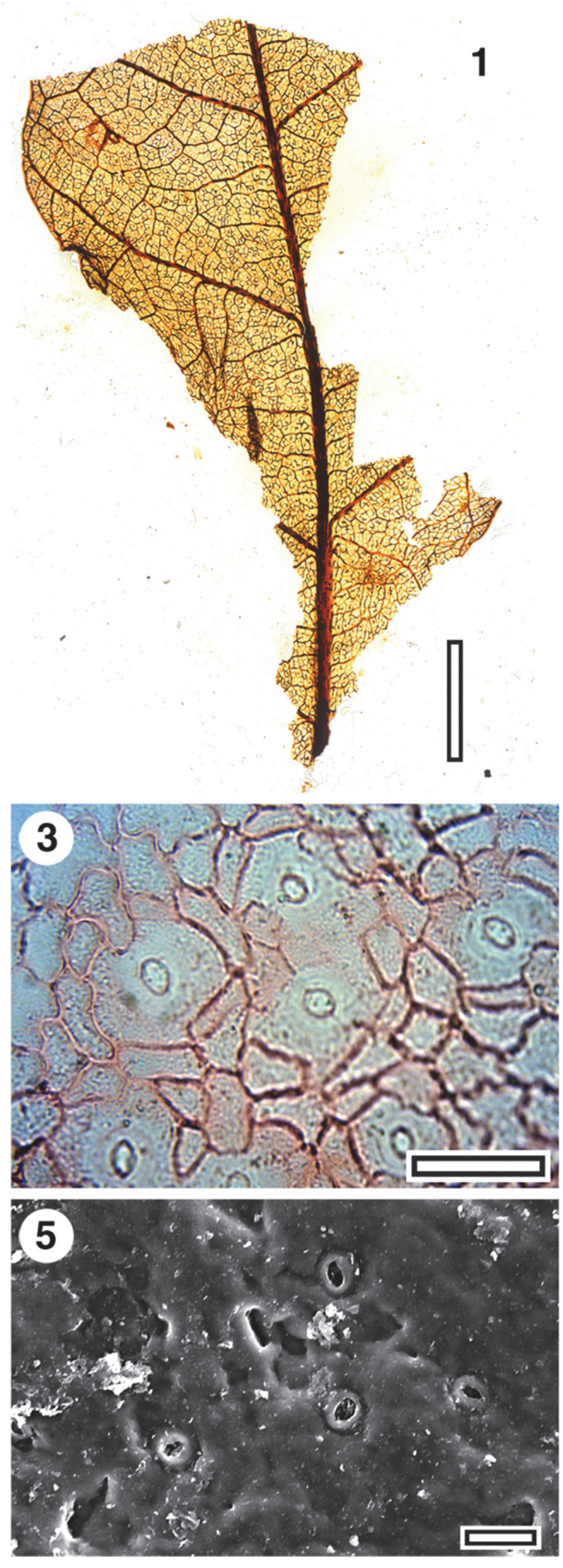
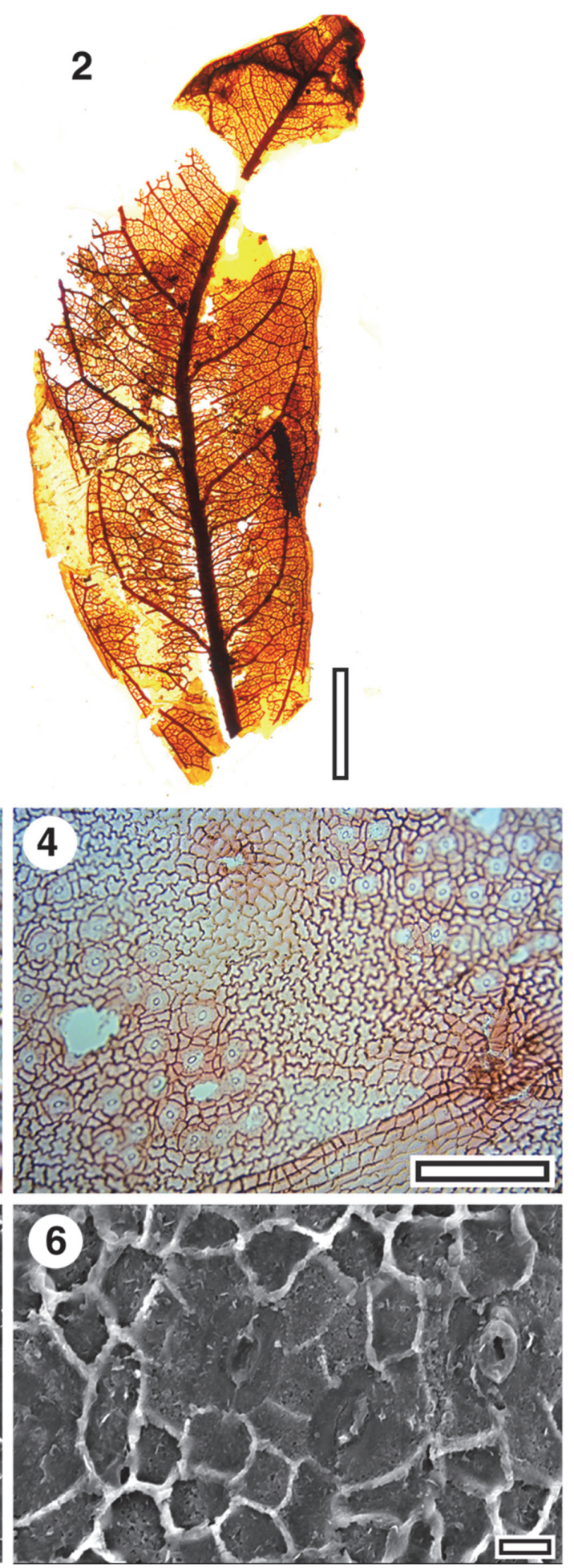

FIGURE 21. MANU-13 and its cuticle CUT-Z-CCE (Mata-01). 1. Glycerine jelly/plastic sheet mount, SL1212 (scale bar equals $10 \mathrm{~mm}$ ). 2. Glycerine jelly/plastic sheet mount, SB1237 (scale bar equals $10 \mu \mathrm{m}$ ). 3. TLM of cuticle with six stomatal complexes (SL1212, scale bar equals $50 \mu \mathrm{m}$ ). 4. TLM of cuticle with two stomatal areas and a major venal area at bottom right. The cells in contact with the guard cells are not clearly distinct. Two possible trichome bases are at top and right (SL1212, scale bar equals $200 \mu \mathrm{m})$. 5. SEM of outer cuticle surface. Four stomatal complexes are in view with simple outer stomatal ledges (S-1952, scale bar equals $20 \mu \mathrm{m})$. 6. SEM of inner cuticle with three stomatal complexes, two of them are sharing contact cells (S-1952, scale bar equals $10 \mu \mathrm{m})$. 


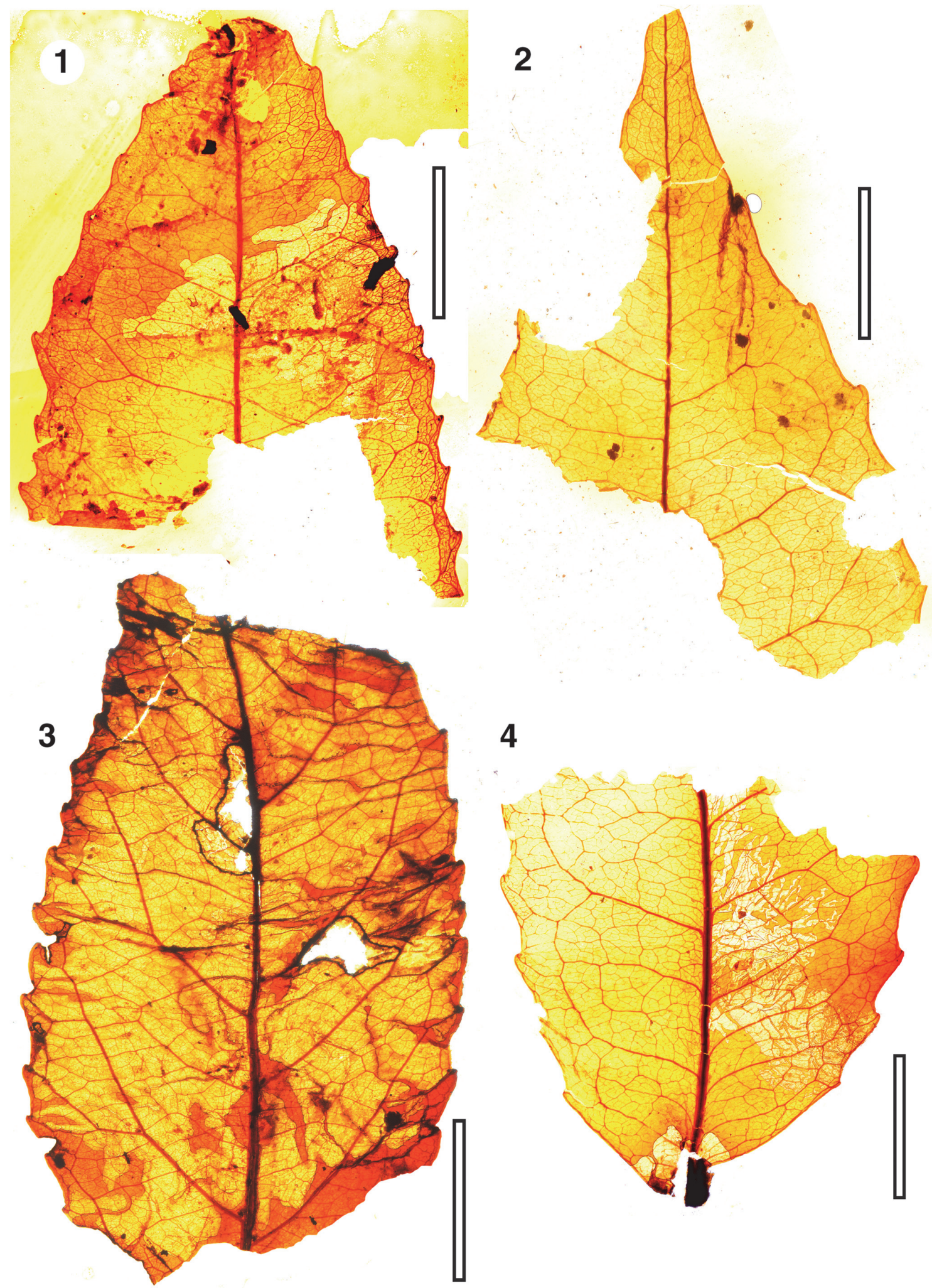

FIGURE 22. MANU-49 (BL-15). Leaves in glycerine jelly/plastic sheet mounts (scale bars equals $10 \mathrm{~mm}$ ). 1. OU29799. 2. OU29800. 3. OU30222. 4. OU29798. 

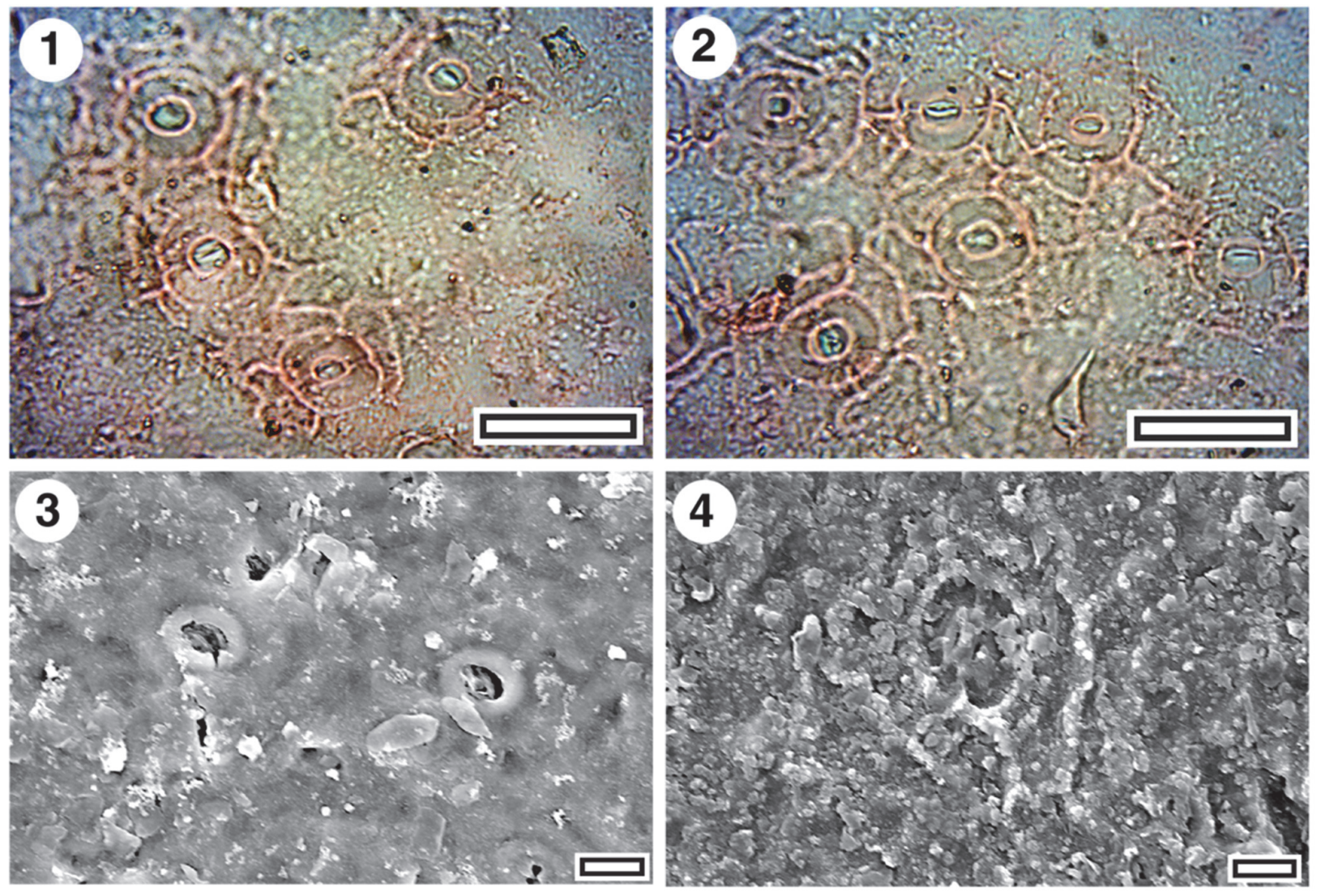

FIGURE 23. CUT-Z-ADF - cuticle of MANU-15 (BL-15). 1. TLM (OU29799, scale bar equals $50 \mu \mathrm{m}$ ). 2. TLM (OU29799, scale bar equals $50 \mu \mathrm{m}$ ). 3. SEM of outer cuticle surface with several stomatal complexes with simple outer stomatal ledges (S-1950, scale bar equals $10 \mu \mathrm{m})$. 4. SEM of inner cuticle surface with a single stomatal complex (S-1950, scale bar equals $10 \mu \mathrm{m})$.

Notes:. A single leaf has relatively bland venation externodromous venation, with robust higher order venation. No teeth are visible. It might be assumed to be Lauraceae, but the cuticle shows this is not so. There are no other Manuherikia Group parataxa that are strikingly similar (MANU-21 may have a similar shape, but is digitate, and the fine venation details are unknown) so the specimen is regarded as a new parataxon.

\section{Taxon MANU-54 (new)}

Figure 25

Reference specimen. LX1446 (GL-05).

Distinguishing features. Differs from all other Manuherikia Group taxa in having an elliptical lamina with very small teeth (c. $0.2 \mathrm{~mm}$ high).

Description. Size: length $70 \mathrm{~mm}$, width $22 \mathrm{~mm}$, microphyll. Shape: elliptical, symmetrical, apex unknown, base convex. Petiole unknown. Margin toothed. First order venation pattern probably externodromous. Development normal. Midrib c.
$1.5 \%$ width of mid-lamina. First order lateral veins probably numbering about five on each side of midrib, not decurrent on midrib, irregularly spaced (c. $10 \mathrm{~mm}$ ), course curved. Teeth very small (c. 0.2 $\mathrm{mm}$ high), little more than slightly hooked (?glandular) extensions of the margin (c. every 2-3 mm).

Cuticle. CUT-Z-EHI (Pole 2008).

Notes. A single specimen has minute teeth, and indistinct but relatively fine venation. The cuticle was very fragmentary and difficult to prepare.

\section{Taxon MANU-55 (new)}

Figure 26

Reference specimen. LX1973 (BL-32).

Distinguishing features. Differs from all other Manuherikia Group taxa in having craspedodromous first order venation and robust, slightly spinose teeth.

Description. Size: length $70-$ possibly $120 \mathrm{~mm}$, width 29-64 mm, microphyll-notophyll. Shape: probably elliptical-obovate, possibly asymmetrical, 

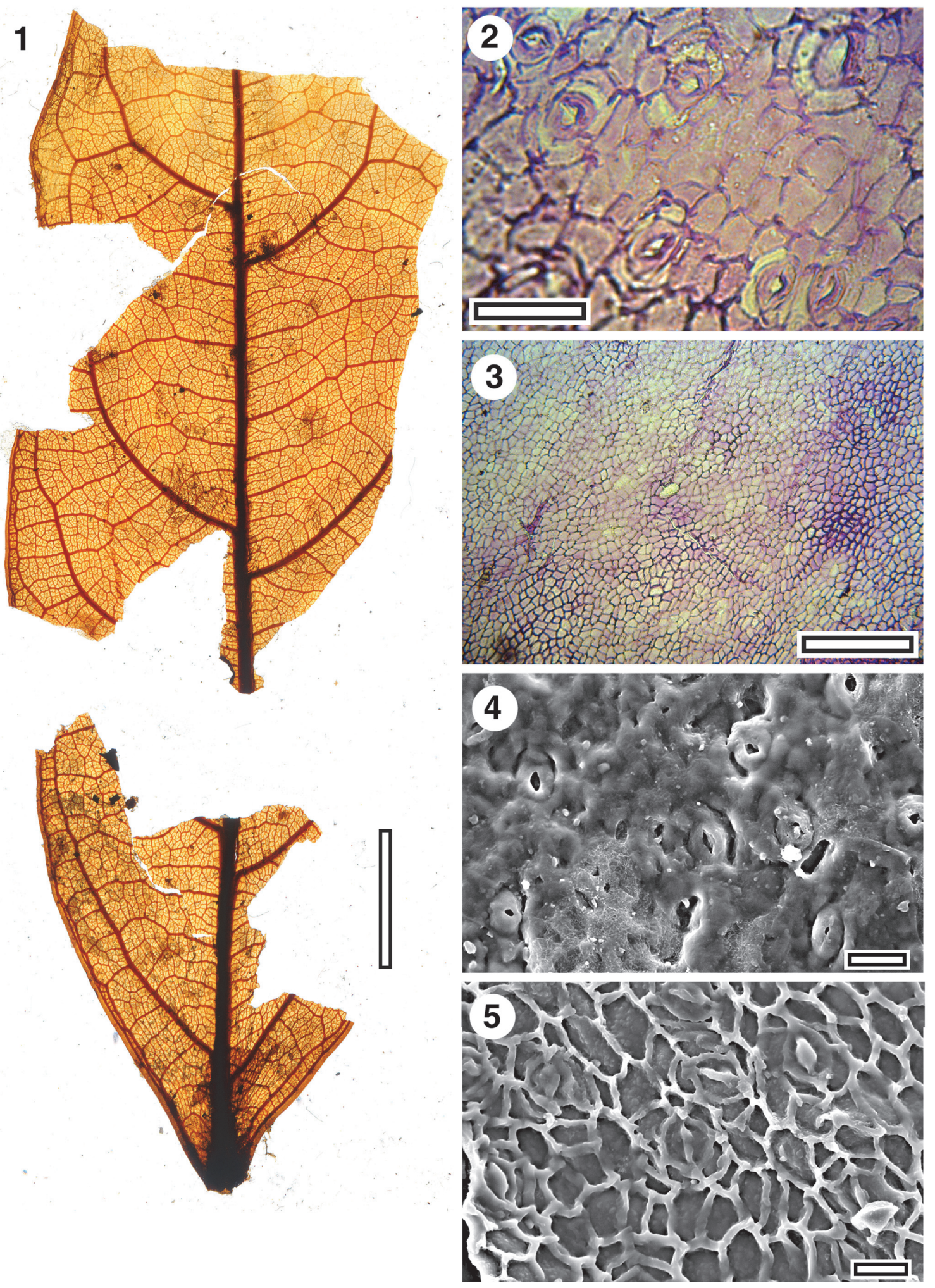

FIGURE 24. MANU-52 and CUT-Z-AJI (OU29848, BL-15). 1. Whole leaf, whole leaf, glycerine jelly/plastic sheet mount (scale bar equals $10 \mathrm{~mm}$ ). 2. TLM of cuticle (scale bar equals $50 \mu \mathrm{m}$ ). 3. TLM of upper cuticle (scale bar equals $200 \mu \mathrm{m}$ ). 4. SEM of outer cuticle surface with several stomatal complexes and very irregular surface (S-1947, scale bar equals $20 \mu \mathrm{m}) .5$. SEM of inner cuticle (S-1947, scale bar equals $20 \mu \mathrm{m}$ ). 

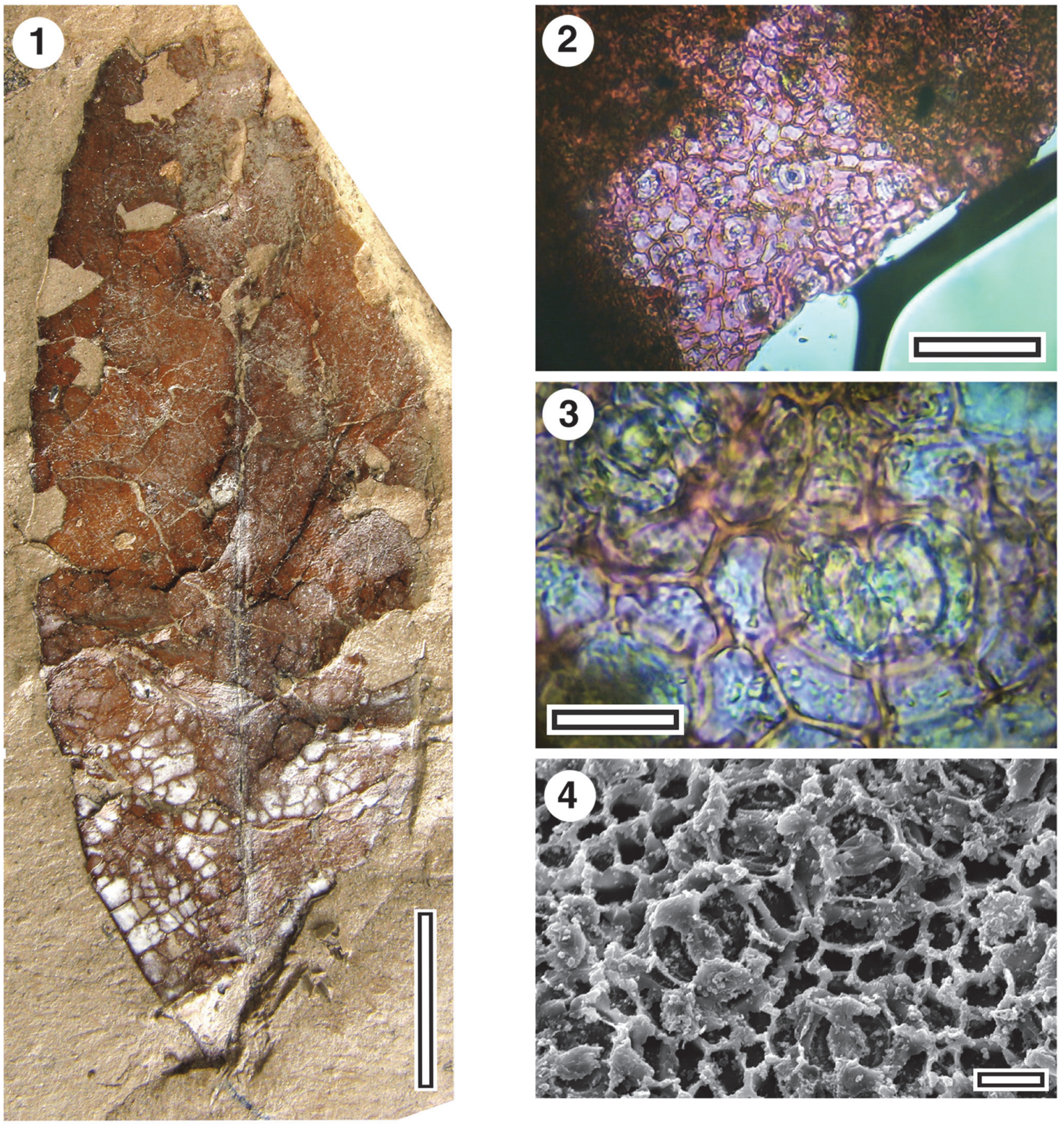

FIGURE 25. MANU-54 and CUT-Z-EHI (LX1446, GL-05). 1. Whole leaf, in situ on mudstone (scale bar equals 10 $\mathrm{mm}$ ). 2. TLM with several stomatal complexes (scale bar equals $200 \mu \mathrm{m}$ ). 3 . TLM of single stomatal complex. The outer stomatal rim is clearly visible, as is the outer bound of the subsidiary cells (scale bar equals $50 \mu \mathrm{m}$ ). 4 . SEM of inner cuticle surface. Prominent T-pieces and cutinisation around the guard cells are apparent (S-1908, scale bar equals $20 \mu \mathrm{m})$.

apex unknown, base unclear. Petiole unknown. Margin toothed. First order venation pattern craspedodromous. Development normal. Midrib c. 1-2 \% width of mid-lamina. First order lateral veins probably numbering at least six to seven on each side of midrib, not decurrent on midrib, irregularly and broadly spaced (c. every 7-11 mm), course curved to straight, angle of divergence c. $45-90^{\circ}$, angle of divergence moderate to wide acute. Basal laterals not paired. Second order venation pattern loosely percurrent. Third order and higher venation pattern unclear. Teeth robust (c. $2 \mathrm{~mm}$ high), slightly spinose, sinusues smooth.

Cuticle. CUT-Z-ABD of Pole (2008). 

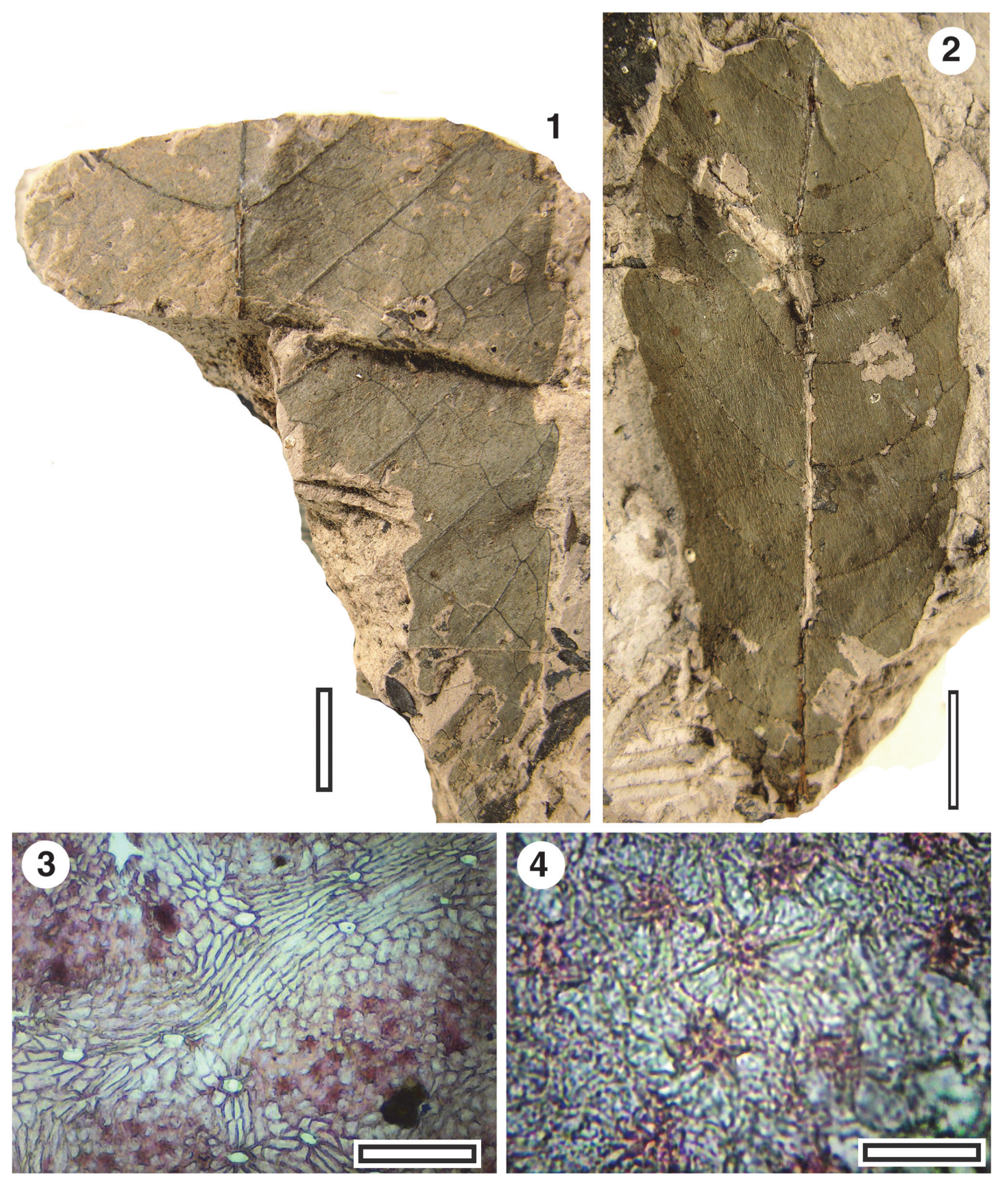

FIGURE 26. MANU-55 and CUT-Z-ABD. 1. In situ leaf on mudstone (SL4855, BL-32, scale bar equals $10 \mathrm{~mm}$ ). 2. in situ leaf on mudstone (SL4856, BL-32, scale bar equals $10 \mathrm{~mm}$ ). 3. TLM of cuticle showing stomatal groups separated by venal areas (SL4856, BL-32, scale bar equals $200 \mu \mathrm{m}$ ). 4. TLM of cuticle (SL4856, BL-32, scale bar equals $50 \mu \mathrm{m}$ ). 


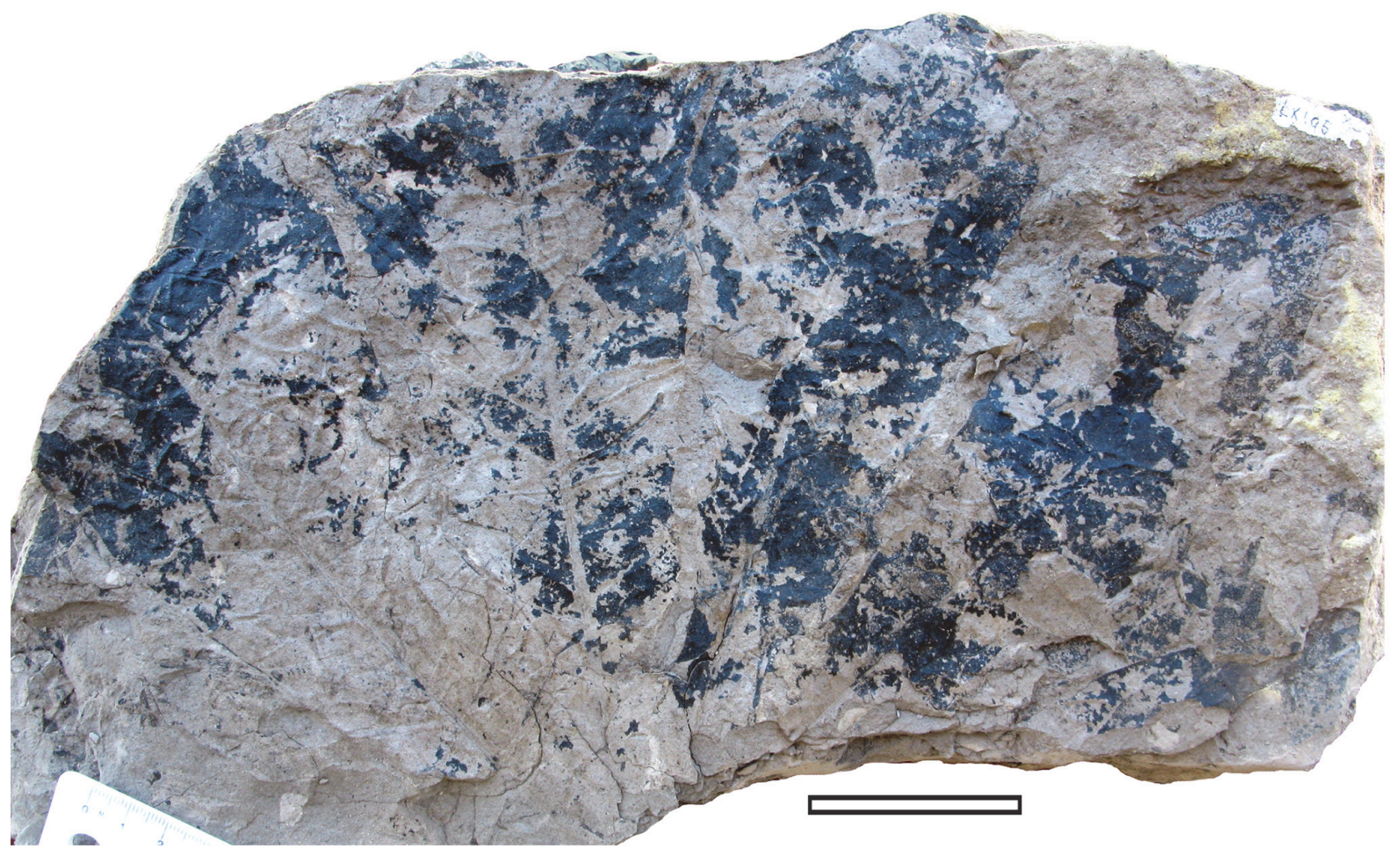

FIGURE 27. MANU-56. Digitately compound leaf, in situ on mudstone. (LX195, GL-01, scale bar equals 50 mm).

Notes. Two leaves have craspedodromous venation, with veins running either relatively straight, or arching to robust teeth (perhaps slightly spinose). The taxon is clearly distinct from other Manuherikia Group craspedodromous taxa (MANU-6/Nothofagus and MANU-23) and is regarded as a new parataxon.

\section{Taxon MANU-56 (new)}

Figure 27

Reference specimen. LX195 (GL-01).

Distinguishing features. Differs from all other Manuherikia Group taxa in being digitately compound with mesophyll-sized leaflets.

Description. Construction, digitately compound. Size: leaflet length c. $220-250 \mathrm{~mm}$, width c. 75-90 $\mathrm{mm}$, mesophyll. Shape: probably obovate, apex unknown, base probably acute. Petiole unknown. Margin entire. First order venation pattern externodromous. Development normal. Midrib c. $2 \%$ or less width of mid-lamina. First order lateral veins probably numbering at least six to seven on each side of midrib, not decurrent on midrib, relatively thin, irregularly and broadly spaced (c. every 7-10 $\mathrm{mm}$ ), course curved, angle of divergence c. 40$50^{\circ}$. Basal laterals not paired, parallel, angle of divergence moderate to wide acute. Lateral loops indistinct. Finer venation unclear.

Notes. A single specimen is interpreted as at least four leaflets of a digitately compound leaf. Unfortunately the bases and apexes are missing. The margins appear to be entire and the venation indistinct, but robust externodromous. The individual leaflets have one of the largest lamina of any Manuherikia Group taxa. For comparison, MANU-18 has widths to at least $165 \mathrm{~mm}$. The only taxon known to be digitately compound, MANU-21, is much smaller $(10-23 \mathrm{~mm})$. The specimen is therefore regarded as a new parataxon.

Cuticle. Unknown.

$$
\text { Taxon MANU-59 (new) }
$$

Figure 28

Reference specimen. LX1437 (GL-05).

Distinguishing features. Differs from all other Manuherikia Group taxa in having teeth restricted to the apical two-thirds of the lamina.

Description. Size: length unclear, probably around $65 \mathrm{~mm}$, width $38 \mathrm{~mm}$, microphyll. Shape: probably obovate, apex widely acute, base acute. Midrib $<1$ $\%$ width of mid-lamina. Margin toothed. Teeth restricted to the apical two-thirds of the lamina, apically pointed, irregularly spaced (c. $1 \mathrm{~mm}$ apart) 

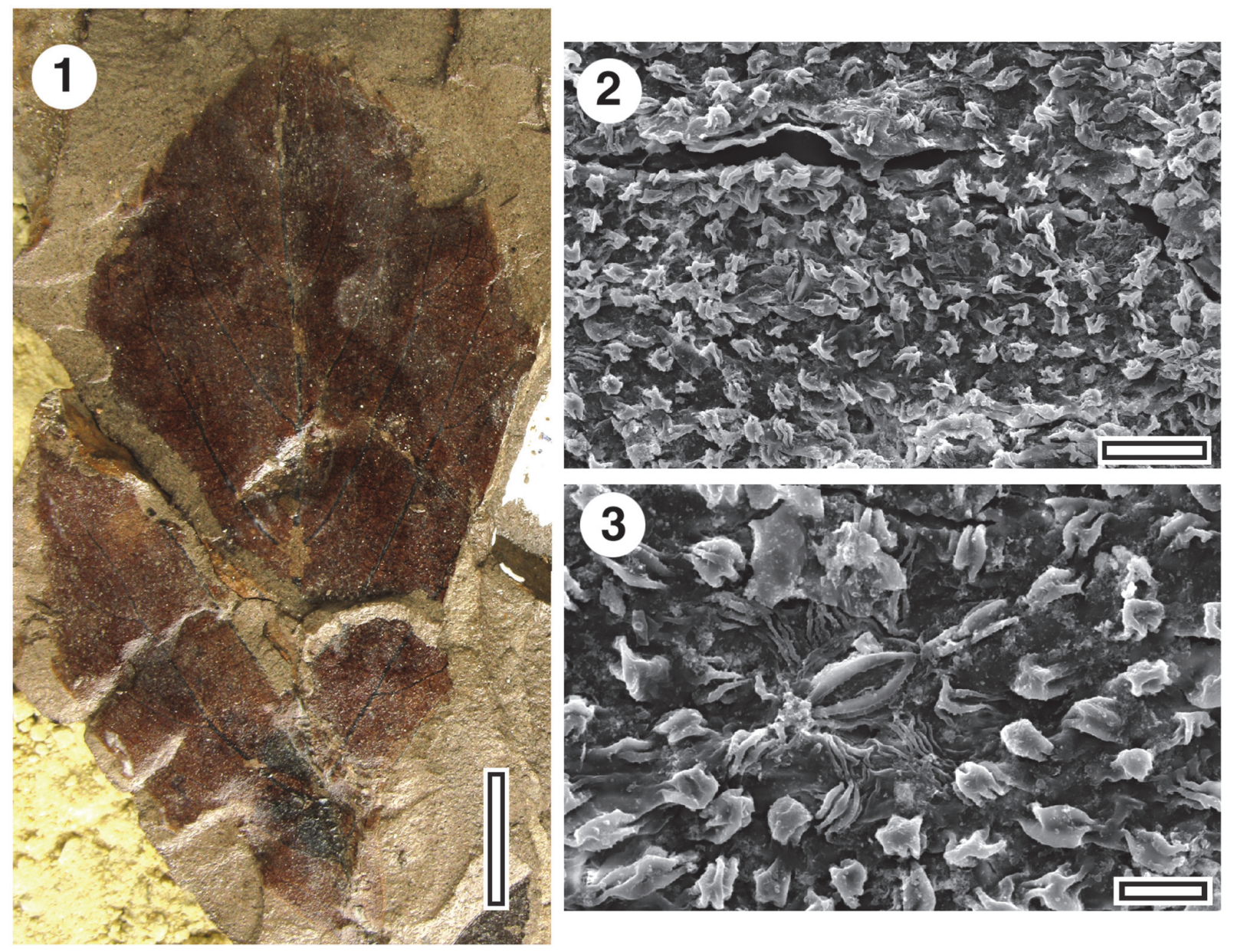

FIGURE 28. MANU-59 (LX1437, GL-05). 1. Whole leaf, in situ on mudstone (scale bar equals $10 \mathrm{~mm}$ ). 2. SEM of outer cuticle (S-1897, scale bar equals $50 \mu \mathrm{m}$ ). 3. SEM of outer cuticle surface showing single stomatal complex (S1897, scale bar equals $20 \mu \mathrm{m}$ ).

and sized (c. $1 \mathrm{~mm}$ high). Venation probably semicraspedodromous, but unclear.

Cuticle. Papillate, with prominently flanged papillae. Stomata are sharply elliptical with a pattern of fine ridges radiating away from the outer stomatal ledges. This cuticle is differs from other papillate cuticle from the Manuherikia Group, but as it is only known from a single fragment on an electron microscope stub (other preparations failed) a new parataxon is not described at this stage.

Notes. MANU-59 differs from all other toothed Manuherikia taxa in its lamina shape and size and shape of its teeth. MANU-30 shows a similar shape, but the teeth and venation are more robust. MANU-16 can have a similar lamina shape, but the margin is more crenate.

\section{Taxon MANU-60 (new)}

Figure 29
Reference specimen. LX272 (GL-05).

Distinguishing features. Differs from all other Manuherikia Group taxa in having a widely elliptical lamina with a few (6-7 up each side), widelyspaced (6-7 $\mathrm{mm}$ apart) teeth.

Description. Size: length unclear, probably around $60 \mathrm{~mm}$, width $36 \mathrm{~mm}$, microphyll. Shape: widely elliptical, apex rounded, base unknown. Midrib c. $1.2 \%$ width of mid-lamina. Margin toothed. Venation unclear. Teeth robust, apically pointed, c. 1.0 $\mathrm{mm}$ high, about 6-7 up each side of leaf, widelyspaced, spaced 6-7 mm apart.

Cuticle. CUT-Z-ECF of Pole (2008).

Notes. MANU-60 differs from all other Manuherikia Group taxa in its widely-spaced teeth and rounded apex. MANU-27 is only known from a small fragment but MANU-60 shows no sign of the prominent loops of MANU-27. 

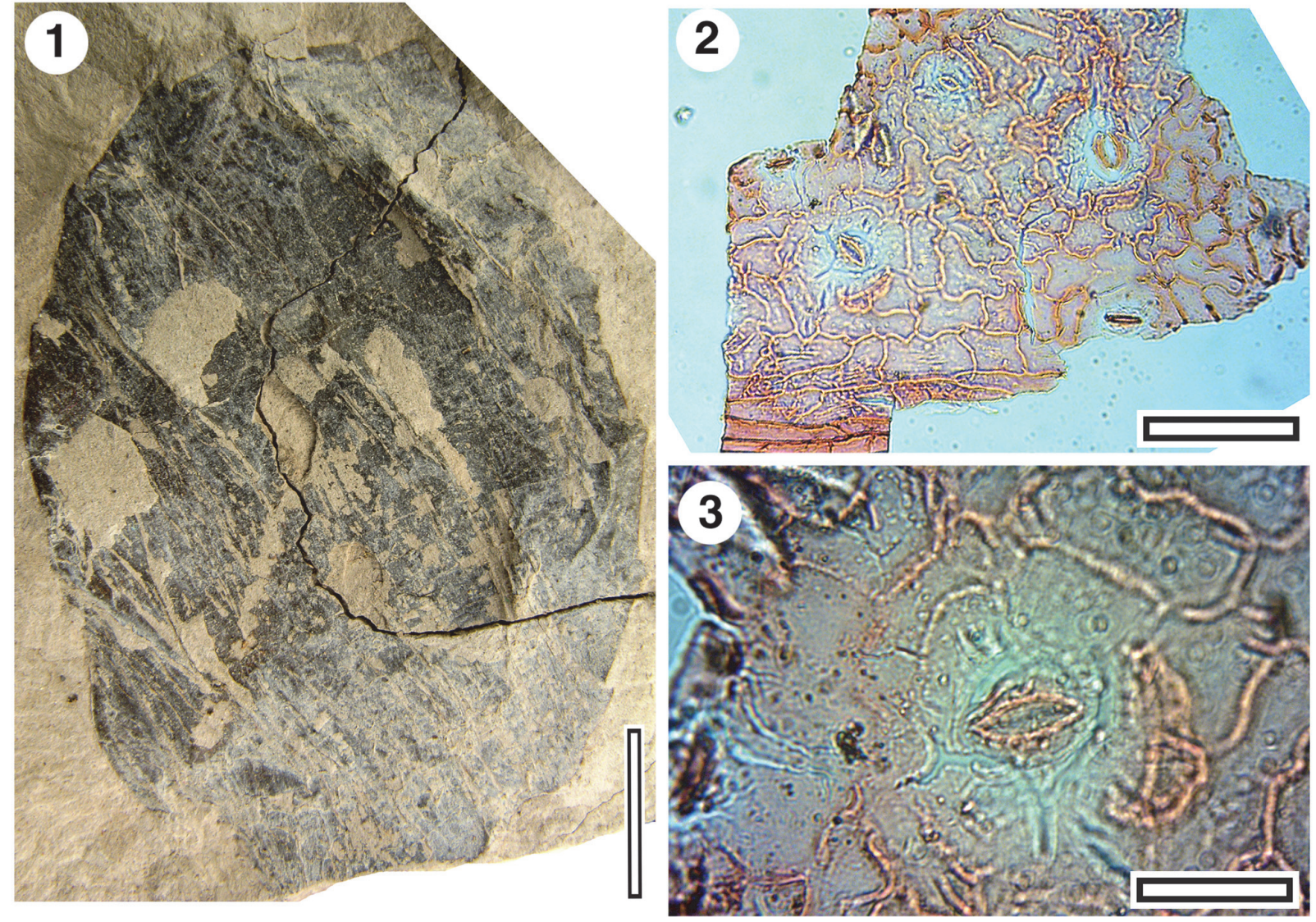

FIGURE 29. MANU-60 and CUT-Z-ECF (LX272 GL-01). 1. Whole leaf, in situ on mudstone, (scale bar equals $10 \mathrm{~mm}$ ). 2. TLM of cuticle (scale bar equals $200 \mu \mathrm{m}$ ). 3. TLM of single stomatal complex (scale bar equals $50 \mu \mathrm{m}$ ).

\section{Lauraceae wood}

Figure 30

Description. Wood having intervessel pits which are alternate and bordered. Rays narrow (one cell), c. 10-15 cells in height, sometimes associated with oil cells. These features are consistent with Lauraceae (Meylan and Butterfield, 1978; Leisman, 1986; Poole et al., 2000). This specimen and other wood from the St. Bathans Member is the subject of on-going research.

\section{Fungi}

Figure 31

Specimens and occurrence. SL3134, GL-02; SL3251, GL-31

Notes. Fragments of leaf cuticle occasionally have well-preserved epiphyllous fungal remains. Branched mycelia are associated with orbicular and elongate hyphopodia. Comparable fossil material is illustrated by Dilcher (1963), Phipps and Rember (2004), Bannister et al. (2016) and Conran et al. (2016).
Roots

Figure 32

Specimens and occurrence. SL2857, GL-18; SL3149, GL-01; SL3180, BL-01; SL3199, BL-32; SL3283, BL-05; SL4785, BL-04; SL4795, GL-25.

Notes. Apparent sections of roots are present in several samples which have six or seven parallel rows of tubercles/rootlets running along their lengths. Holdgate et al. (2007, fig 9c) illustrated 'proteoid roots' from the middle Miocene Yallourn coal seam in Victoria. Their material is broadly comparable, but does not appear to have the distinct rows or rootlets.

\section{DISCUSSION}

\section{The Paleoclimate}

Paleoclimate of the southern New Zealand Miocene has been summarised by Pole (2014), and this included leaf-length analyses for St. Bathans material (Further leaf-size histograms are given here, Figure 6). Most leaves fall into the 

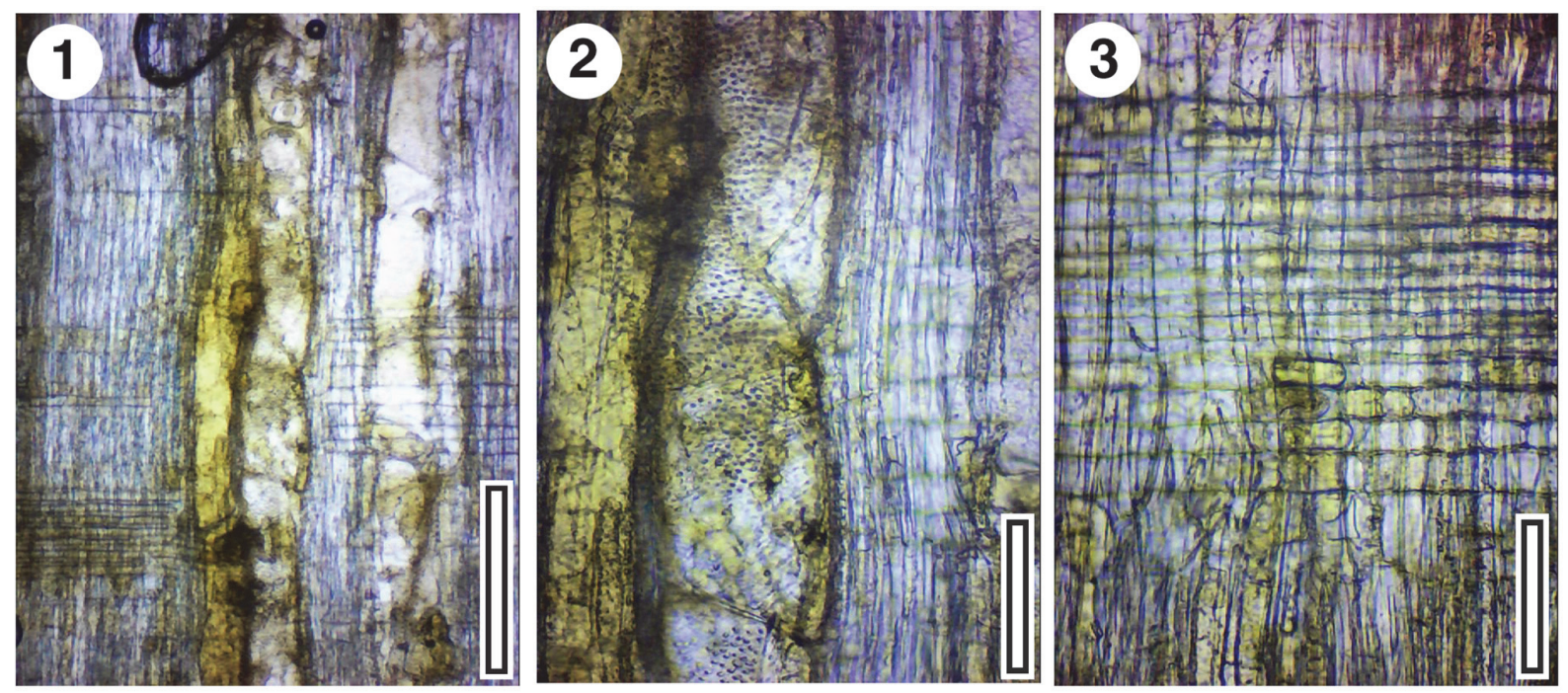

FIGURE 30. Lauraceae wood (LX1977, GL-34). TLM images of radial sections. 1. Vessels and rays (scale bar equals $0.1 \mathrm{~mm}$ ). 2. Detail of vessel showing alternate pitting (scale bar equals $20 \mu \mathrm{m}$ ). 3. Detail of ray showing resin-filled cells (scale bar equals $20 \mu \mathrm{m}$ ).

microphyll class and fewer into the notophyll. Leaf size, average length (following Greenwood, 1992; Carpenter et al., 1994a) and floristic composition (Pole, 2014) suggest Mean Annual Temperatures (MAT) of about $14-20^{\circ} \mathrm{C}$, that is, 'warm-temperate' following widespread usage (e.g., Pocknall, 1989) or 'subtropical' following Wolfe (1979, warmer temperatures would be 'paratropical') or 'mesothermal' following the non-geographic system of Nix (1982). Today St. Bathans lies close to $45^{\circ} \mathrm{S}$, but was likely around three degrees higher in the early Miocene (using GPlates, http://www.earthbyte.org, with the Müller et al., 2008 rotation model). The suggested paleo-MATs are thus $3-9{ }^{\circ} \mathrm{C}$ warmer than maritime temperatures at that latitude today.

Foliar physiognomic analysis ('CLAMP' and Leaf Margin Analysis; Wolfe, 1979, 1993) was not attempted for St. Bathans, as there are too few taxa known from intact leaves. However, foliar physiognomic and co-existence analyses for other (younger) Manuherikia Group assemblages and for southern New Zealand early Miocene in general, arrive at similar results - at least $17-18^{\circ} \mathrm{C}$ (Pole, 2014). Frosts, if they occurred, were likely mild and few. The taxa present all suggest ever-wet conditions although rainfall amounts may have been
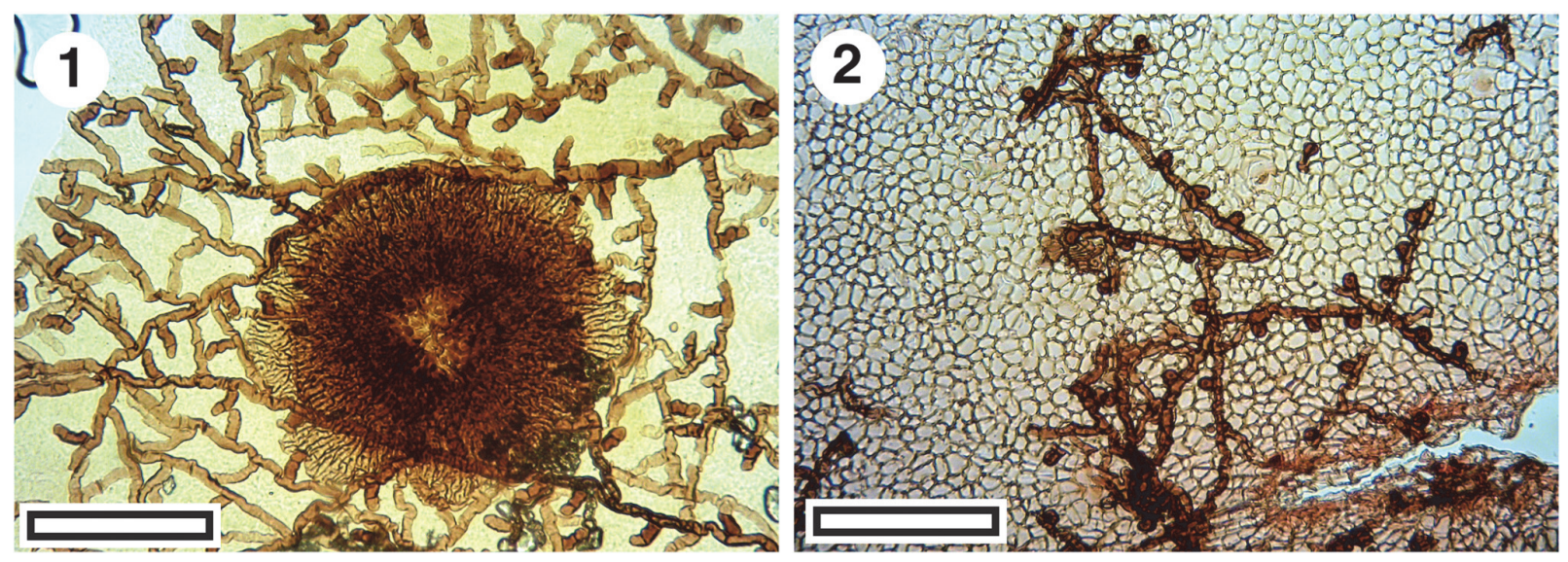

FIGURE 31. Epiphyllous fungi (TLM images). 1. Circular stromata (ascomata), cf. Phragmothyrites sp, and branched mycelia associated with elongate hyphopodia (SL3251, GL-31, scale bar equals $200 \mu \mathrm{m}$ ). 2. Branched mycelia associated with orbicular hyphopodia (SL3134, GL-02, scale bar equals $200 \mu \mathrm{m}$ ). 

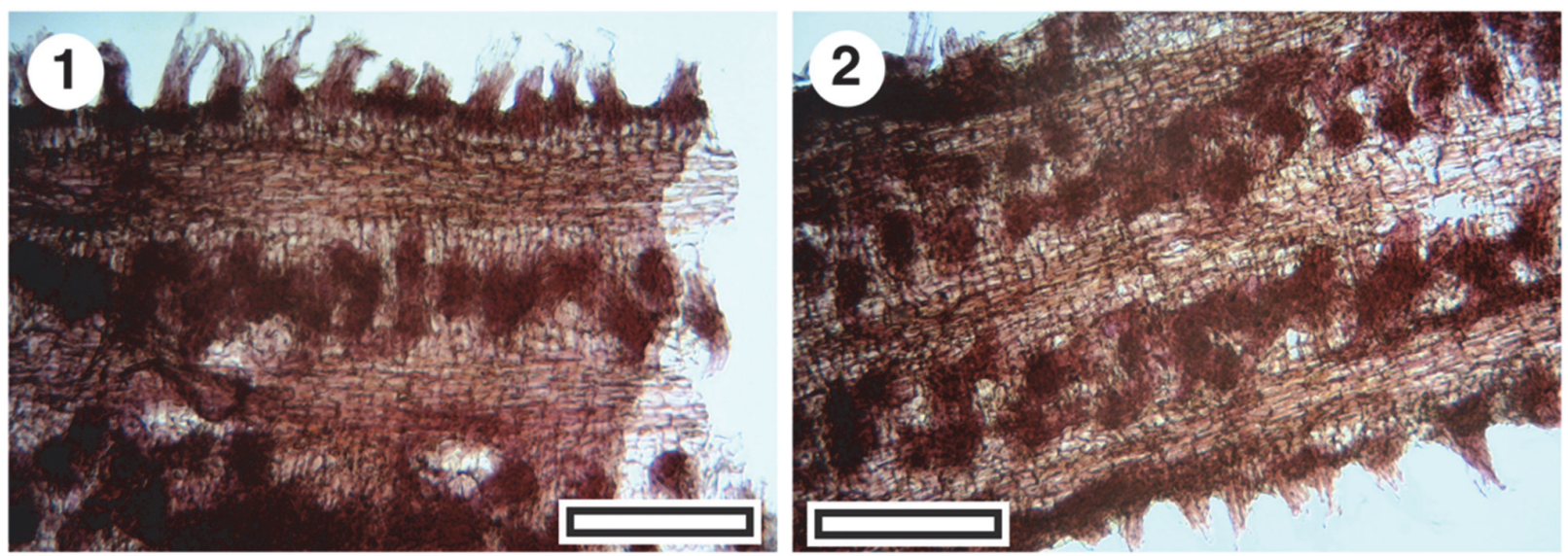

FIGURE 32. Roots, TLM images, showing parallel zones of lateral rootlets, scale bars equals $0.5 \mathrm{~mm}$ ). 1. SL4795, GL-25. 2. SL4795, GL-25.

highly variable to account for the braided fluvial system.

A range of paleoclimate estimates for the Manuherikia Group, including one for the St. Bathans Palaeovalley, were offered by Reichgelt et al. (2015). These were based on 'leaf morphotypes', with 10 of these illustrated as photographs from the GL-05 locality. The basis on which those morphotypes were distinguished is unclear - they are not described and primary CLAMP data were not supplied. A further 12 morphotypes were listed that had their 'types' in other Manuherikia Group localities and which were also defined solely on the basis of a photograph. As the basis of these morphotypes, Reichgelt et al. (2015) figured specimens that had already been published as both figures and descriptions in Pole (1993b, 1993c, 1993d) (their Morphotaxon D-figured by Pole 1993c, fig 7c; Morphotaxon U-figured by Pole 1993a, fig 5d; Morphotaxon V-figured by Pole 1993c, fig 23; Morphotaxon AT-figured by Pole 1993c, fig 26a; Morphotaxon AY-figured by Pole 1993c, fig 13a; Morphotaxon BC-figured by Pole 1993c, fig 24n).

Three of the other four leaf fossil assemblages studied by Reichgelt et al. (2015) were collected previously: two from Bannockburn (registered with the New Zealand Geological Society Fossil Record database as F41/f220 and F41/ f235) and one from Lauder Station (H41/f046). Despite being provided with fully curated collections, they made no reference to any of the existing published descriptive work on those specimens (Pole, 1993a, 1993b, 1993c, 1993d, 1993e, and a key to distinguish all published Manuherikia Group leaf parataxa, Pole, 2014). Reichgelt et al. (2015) simply listed some of the papers in a Supplementary file but with no mention of them in the text. Instead, Reichgelt et al. (2015) used morphotypes based only on images. No explanation was given as to why they were different from each other, and more importantly, the criteria by which specimens in other assemblages could be assigned to a morphotype. The resulting datasets are substantially different from what has been previously published on the same material. Pole (1993f, and references therein), documented seven taxa from the F41/ f235 assemblage, while Reichgelt et al. (2015) claim 20 but only cite (and figure) nine specimens from that assemblage. For the H41/f046 assemblage, Pole (1993f) documented three taxa, versus 21 taxa claimed, and nine specimens cited by Reichgelt et al. (2015). For these two assemblages, the Reichgelt et al. (2015) CLAMP climate estimates are based not only on taxa without descriptions, but mostly on taxa for which specimen numbers are not given and which are assigned to morphotypes in other localities based on unstated criteria.

Furthermore, Reichgelt et al. (2015) mislocated all of the previously collected and curated leaf fossil assemblages to other sites several kilometers away. The two assemblages from Bannockburn (F41/f220 and F41/f235) were indicated on a measured section of the Kawarau River, which was redrawn from a PhD thesis (of B.J. Douglas) without acknowledgement (confirmed in letter to Pole from D.E. Lee, 09.07.18). They showed the Lauder Station assemblage ( $\mathrm{H} 41 / \mathrm{f0} 46)$ on a measured section of Vinegar Hill, which is not only misplaced laterally, but stratigraphically - the carbonaceous Fiddlers Member fossil assemblage was indicated 
as coming from unfossiliferous lacustrine Bannockburn Formation. Reichgelt et al. (2015) also provide a measured section of the DH1 Nevis DSIR drill core from the Nevis Valley. However, since drilling was completed in 1978, the Nevis core had been mis-curated in the New Zealand Petroleum and Minerals National Core Store until researched in September 2018 by B.J. Douglas (personal commun. 2018). It is unlikely to have been available to Reichgelt et al. (2015). The reasonable inference is that both the Vinegar Hill and Nevis $\mathrm{DH} 1$ sections shown in Reichgelt et al. (2015) also originate in Douglas's thesis (where they were presented in detail).

That is, Reichgelt et al. (2015), a publiclyfunded course of research (a Royal Society of New Zealand Marsden Grant) was provided with three of the five assemblages they studied, but did not reference any of the descriptive publications on those specimens, misplaced all their locations and showed them on measured sections which had been re-drawn from someone else's research without acknowledgement. To obtain their climate estimates, Reichgelt et al. (2015) ignored the existence of a fully described and keyed taxonomy in favour of a series of undescribed morphotypes and where a significant (sometimes the majority) of morphotypes in each assemblage are not backedup by cited specimens. They collected a further locality from the St. Bathans Member (the GL-05 documented here), claimed it as "new", ignoring four taxonomic publications on that horizon alone (Pole, 2007a, 2007b, 2008, 2014). The apparent absolute refusal of Reichgelt et al. (2015) to acknowledge existing taxonomic publications on their specimens or the wider area they are working on, to deal with them in the usual way (by synonymising, splitting, etc.) and instead to use a de novo unverifiable dataset suggests reasonable grounds to disregard their work. That course is followed here.

Prebble et al. (2016) arrived at a MAT centred around c. $19.6{ }^{\circ} \mathrm{C}$, for the New Zealand early Miocene (Otaian-Altonian). Thus their average equates to the upper limit suggested here. Their generally warmer results follow from restricting their modern floral dataset to Australia, New Zealand and New Caledonia. By excluding the montane vegetation of New Guinea, a region specifically identified as a modern analogue of the early Miocene New Zealand (Pocknall, 1989), and also Australia (Duigan, 1966; Kemp, 1978), Prebble et al. (2016) introduce a systematic warming into their results.
Rainfall is likely to have been everwet (Pole, 2014). Additionally, Pole (2003) argued that the subtropical high pressure cells may have typically tracked to the south of New Zealand in the early Miocene. If this was the case, a frequent synoptic situation then may have been what is periodically experienced in the New Zealand summer today - a high pressure cell centred at around $50^{\circ} \mathrm{S}$, with rainfall provided by cyclonic systems developing in the Tasman Sea to the west, or in the south-west Pacific to the north and northeast. Until the distinct Antarctic/Southern Ocean cooling occurred around $14 \mathrm{Ma}$ (Flower and Kennett, 1994), the frontal systems which provide so much precipitation to New Zealand today, were either too far south to effect New Zealand (if they were centred on the location of the 'protoSubantarctic' and 'Subtropical' oceanic fronts in Nelson and Cooke, 2001) or perhaps did not exist at all in their modern sense.

\section{Biodiversity and Soils}

The sediments of the St. Bathans Palaeovalley tend to be either very pure quartz sand and gravel, or mud. The regions of pure quartz sand (exposed bars) would have been an extremely lownutrient and freely-draining substrate difficult for plants to colonise. These conditions bring to mind the white-sand of the 'black-water' rivers in the Amazon today (Janzen, 1974, although of likely different river morphology). In the St. Bathans Palaeovalley nutrient levels in the quartz sand locations were probably lower even than the deeply weathered greywacke/schist basement rocks on the hills. The muddy regions (channel-fill, flood-plains) would have had somewhat higher nutrient levels but were probably water-logged. Each of these microenvironments within the valley likely had a quite different plant community. The fact that the fluvial style was braided suggests a highly variable discharge (Douglas, 1986) responding to a variable rainfall. Additionally a braided style would have made life challenging for the vegetation growing within the valley, as it would need to have dealt with often-changing channel patterns, and may even have faced valley-full floods from time to time. Superimposed on the mosaic of soil-types would have been a range of successional-stages as abandoned bars and channels were re-vegetated.

Despite these conditions, and perhaps because of them, the palaeovalley had an extraordinarily high biodiversity. The muddy units in the palaeovalley (that are mostly flood deposits, rather than actual soils) have excellent fossil plant content. From the entire Palaeovalley, the remains of 
161 taxa (145 angiosperms 14 conifers, and two cycad-like gymnosperms) have been published from fragments of leaves and shoots (Pole, 1992, 1993a, 1993b, 1993c, 1993d, 1997, 1998, 2007, 2008a, 2008b, 2008c, 2010, 2014; Hill and Pole, 1994; Pole et al., 2003, 2008). The highest cuticle diversity was found in BL-32. From this one sample, the leaves and shoots of 48 angiosperms and seven conifer taxa have been recovered. The most widespread taxa are CUT-Z-ABD (in 38 samples), Retrophyllum (31), CUT-L-ECB (30), Dacrycarpus (in 26 samples), and CUT-M-DID and CUT-P-022 (both in 27 samples). Pole (2007) noted a relationship between extant Lauraceae species diversity and local total tree diversity. Based on this, the number of Lauraceae taxa known from the Palaeovalley (21) suggests a tree diversity of about 120 . The 161 fossil taxa that have been found is broad confirmation - the additional taxa are likely to have been shrubs and lianes.

As the dispersed cuticle total would have come only from those trees, shrubs and lianes with a robust cuticle, the total diversity would have been astounding. Data from palynology increases the total even more, with monocots and ferns that have not been recovered as macrofossils. To place this diversity in to a recent context, McGlone et al. (2010) recognised 215 species of tree ( $\geq 6 \mathrm{~m}$ tall) in the entire New Zealand region (including the subtropical Kermadec Island). In northernmost New Zealand Burns (1995) found a mean of about 30 trees, shrubs and lianes from patches (0.02-0.04 ha) and a maximum of 52 species.

This makes the St. Bathans Palaeovalley one of the most diverse Cenozoic fossil floras ever published. Christophel and Blackburn (1978) estimated a diversity of approximately 200 species from the Australian Eocene location of Maslin Bay, although to date only four Maslin Bay taxa have been published (Blackburn, 1981; Carpenter et al., 2006). The Oligocene Little Rapid River deposit in Tasmania has produced 26 conifer species in 14 genera (Hill and Brodribb, 1999). More recently, Wilf et al. (2005) have described 152 species of leaf from the Eocene of Laguna del Hunco and Rio Pichileufu in Patagonia, Argentina. In the USA, 347 leaf morphotypes are known from the Cretaceous and Paleogene of the Hell Creek Formation and Ludlow Member of the Fort Union Formation (75 taxa from just the Paleocene) (Johnson, 2002).

Thus the biodiversity of the palaeovalley may not have been unusual for Cenozoic times - other fossil deposits around the world with as good fossil preservation are likely to have similar or even higher diversity. Despite the overall high diversity in the St. Bathans Palaeovalley, there are samples with a notably low diversity. For example, there are seven samples (BL-17, 19, 21, 26, 27, GL-19, 31) with five or less taxa but which include Retrophyllum and Myrtaceae. It is speculated that this Retrophyllum-Myrtaceae combination may be a lowdiversity association that was wide-spread across the valley, perhaps in response to a level of disturbance from floods and shifting channels that hindered rather than boosted biodiversity.

The composition of BL-30 has previously been noted as distinct (Pole, 2007), and was speculated to result from climate change. BL-30 is a typical lens of carbonaceous mud with intact leaves, yet it contains almost the only Dacrydium, Papuacedrus, and the small-leaved Podocarpus alwyniae macrofossils, and, despite it being the most common conifer overall - no Retrophyllum. The composition suggests relatively cool conditions, and the assemblage might represent vegetation normally at higher locations along the valley.

The variety of taxonomic composition in the different samples is consistent with the mosaic of vegetation communities that would have occurred across the palaeovalley. Different compositions would have resulted, for example, from the history of flood events - whether these created bare land surface or simply disturbed existing vegetation, and the time vegetation had to recover from those floods. The quartz gravels of the bars and the mud of the flood basins or abandoned channels would have provided very different habitats with respect to drainage and soil nutrient levels and would have had an important patterning effect on floristics. Podocarp conifers are prominent on the alluvial plains of braided rivers of the West Coast of New Zealand today. Floods that produce large bare surfaces favour the establishment of extensive Dacrycarpus stands, while smaller disturbances (and poorer soils) favour Dacrydium (Wardle 1974, 1977, 1980; Duncan et al., 1990; Norton and Leathwick, 1990; Miller, 2004). In the St. Bathans Palaeovalley, Dacrycarpus dacrydioides was present, and a similar ecology to the West Coast today can be inferred. One sample, BL-25, may reflect a Dacrycarpus-dominant vegetation (Dacrycarpus is the only conifer). However, in most cases Dacrycarpus appears to have been subordinate to Retrophyllum, a genus now extinct in New Zealand. Today Retrophyllum can be found as scattered trees in lowland rainforest in Fiji (Farjon and Page, 1999; pers. obs.) or in New Caledonia as trees growing along the margins of watercourses (Jaffré, 
1995) or even in deep standing water (Hope and Pask, 1998). The latter ecology may well be a model for the St. Bathans Palaeovalley Retrophyllum.

Despite the prominence of quartzose gravel and sand in the St. Bathans Member, the conifer flora tends to be those genera, which are now associated with relatively 'fresh' soils in New Zealand (McGlone, 1988; Pole, 2007). The genera which are more common on the older, and more leached soils, such as Dacrydium, are not common. This might reflect the dynamic environment of the SBM where shifting channels kept the substrate fresh, even though much of it may have been based on low-nutrient quartz gravels and did not allow the accumulation of acidic peat swamps.

The exposure with GL-30, 31, 32, and 33 is interpreted as a lake that formed in an abandoned channel. Periodic floodwaters built up thin beds of mud and initially brought in small fragments of monocot swamp vegetation that established around the lake edge. This was replaced by swamp forest, and intact broadleaves were washed into the lake. Gradually Retrophyllumdominated swamp forest expanded and finally grew over what remained of the lake. This vegetation was terminated when a fluvial channel scoured through the area, and then deposited gravel.

The accumulation of wood in GL-34 includes fragments that are consistent with Lauraceae, which fits with the prominence of Lauraceae in the leaf/cuticle record.

\section{The Age of the St. Bathans Palaeovalley}

The St. Bathans Member is stratigraphically the oldest unit of the Manuherikia Group (Douglas 1986). It was not included in Mildenhall and Pocknall's (1989) palynological study of the Manuherikia Group, although Pocknall (1982) presented a pollen list for a sample from Blue Lake. It listed Proteacidites stratosus Pocknall and Mldenhall (1984), a species that initially helped define the top of the $P$. isopogiformis in Pocknall and Mildenhall (1984), but its range was later extended through the $S$. latispinosus Zone by Mildenhall and Pocknall (1989). The basic palynological 'signature' reported by Pocknall - outright dominance by Nothofagus of the "brassii" type, (62\%), but much rarer "fusca" type $(5 \%)$ and Podocarpaceae $(2 \%)$ compares with assemblages that Couper (1960, fig. 3) regarded as Waitakian, such as the east bank of the Mataura River. This location was placed in the Rhoipites waimumuensis Zone by Pocknall (in Issac et al., 1990), which is probably of Otaian age and close to the Oligocene-Miocene boundary. It differs from the St. Bathans sample in that both $N$. cranwellae and $N$. mataurarensis are dominant (Couper, 1960), whereas Pocknall did not list $N$. matauraensis at all in his St. Bathans sample. Mildenhall and Pocknall (1989) placed most of the older Manuherikia Group sediments they investigated into their Spinozonocolpites latispinosus Zone, although they recognised the older $P$. isopogiformis Zone in just one location - at the base of the Gimmerburn/Haughtons Hill drillhole. The $P$. isopogiformis Zone is probably of Altonian age, as early-mid Altonian marine underlies $P$. isopogiformis Zone lignite at Kapuka in Southland (Pocknall in Issac et al., 1990).

The recognition of the $P$. isopogiformis Zone in what are probably post-SBM, non-incised sediments at Gimmerburn by Mildenhall and Pocknall (1989) indicates the SBM might itself belong to the $P$. isopogiformis Zone, or be even older. However, the current study failed to find reliable critical palynological taxa for placement into the Pocknall and Mildenhall zonation.Tthe key taxa are apparently rare in Central Otago. If this is the case, then perhaps the one instance of the $P$. isopogiformis Zone cited by Mildenhall and Pocknall (1989) may underestimate the real extent of this zone (it is not clear that the other samples placed in to the $S$. latispinosus Zone were all based on actual presence of key taxa). One pollen species found in the present study, Rhoipites titokioides Mildenhall and Pocknall (1989), was listed as restricted to the $S$. latispinosus Zone by Mildenhall and Pocknall (1989), but its presence in the SBM, suggests that $R$. titokioides ranges earlier than the $S$. latispinosus Zone. The qualitative criteria given by Pocknall and Mildenhall (1989) that might distinguish the Rhoipites waimumuensis Zone from the $P$. isopogiformis Zone in Southland do not seem to be confidently applicable in the SBM. For example, Casuarinaceae pollen is important in both zones in Southland, but is typically uncommon in the SBM. Likewise, Pocknall and Mildenhall (1989) describe Podocarpaceae as not accounting for more than $10 \%$ of the Rhoipites waimumuensis Zone pollen sum, versus regularly accounting for about $10 \%$ of the $P$. isopogiformis Zone pollen sum, while in the SBM, Podocarpaceae is often uncommon (present at only 2-3\% in the two counted samples) and sometimes entirely lacking.

In most SBM samples Nothofagus pollen clearly dominates (i.e., $90 \%$ of the counted sample GL-03) and can be placed into the Nothofagus 'brassii' type Zone of Pole and Douglas (1998). Some samples are dominated by Myrtaceae (e.g., 
GL-05) and fall within the higher Arecaceae-Myrtaceae Zone.

Some dispersed cuticle taxa from the St. Bathans Member are also found in the Southland Lignites, which is at least consistent with them being broadly the same age. Pole (2007) found nine species of Lauraceae parataxa that were present in both St. Bathans and Southland samples. However, there is no evidence that any of those taxa have a stratigraphic distribution that might compliment the Pocknall and Mildenhall zonation. All the dispersed cuticle taxa that are present in more than two or three samples in Southland are present in both the $P$. isopogiformis and S. latispinosus Zones.

The inability to simply link the SBM with the Southland (Pocknall and Mildenhall, 1984) palynostratigraphy might reflect the distinct environment of the SBM. However, two other lines of evidence can at least help to give maximal constraints on the age of the SBM. Firstly, although late Oligocene-earliest Miocene palynological samples in New Zealand are mostly dominated by Nothofagus pollen, there was a regional change during the middle Oligocene (Duntroonian) from dominance by $N$. maturaensis to dominance by $N$. cranwellae (Morgans et al., 2004). As the dominant Nothofagus pollen in St. Bathans samples is $N$. cranwellae, the St. Bathans channels are most likely post this date.

Secondly, the St. Bathans Palaeovalley can be put into a regional landscape context. The Waipounamu Erosion surface is an extensive topographic feature, truncating basement rock that extends over much of southern New Zealand (Landis et al., 2008). It has been interpreted as a wave-cut platform and is dated to maximum transgression of the sea around $24 \mathrm{Ma}$. The SB Palaeovalley lies within the extent of this erosion surface - the Hawkdun Mountains to the north have a prominently flat top, while the Dunstan Mountains to the west are almost as flat, although in neither of these areas is any marine sediment preserved to directly confirm a marine origin of the surface. However, if this origin is accepted, and the SB Palaeovalley is incised into schist/greywacke that was earlier planned off by the erosion surface, then $24 \mathrm{Ma}$ is a maximum age for the palaeovalley. Furthermore, Douglas (1986) interpreted a palaeotopography of up to $100 \mathrm{~m}$ immediately surrounding the SB Palaeovalley, and this topography must have arisen since a $24 \mathrm{Ma}$ planation event. A brief period of tectonism around this time in the region has been proposed (Stein et al., 2011). Incision of the SB Palaeovalley probably followed from the uplift that formed during that period, and at the same time, terminated the regional marine transgression and initiated deltaic sedimentation into the Eastern Southland Lignite Basin (including the Gore Lignite Measures) to the south (Stein et al., 2011). Some of the quartzose gravels in this basin may have been transported there by SB type fluvial systems. Palynological investigation (Pocknall and Mildenhall, 1984) indicates that marine sedimentation (Chatton Formation) along with marginal marine and freshwater swamps (Pomahaka Formation) existed in the inland Eastern Southland region up until at least the Otaian, and that the main phase of lignite formation on a prograding delta (Gore Lignite Measures), began about the same time. Thus incision of the SBP likely dates to around the Otaian and sedimentation within the SBP obviously began sometime after the actual incision. To summarise, it is most likely that the SBP was deposited in the Otaian-Altonian of the early Miocene, and is therefore probably equivalent in age to the Rhoipites waimumuensis and/or the Proteacidites isopogiformis Zone of Pocknall and Mildenhall (1994). See Pole (2014) for a broader discussion of the age of Miocene plant fossil localities in southern New Zealand.

\section{CONCLUSIONS}

The early Miocene St. Bathans Palaeovalley Ecosystem was a response to rejuvenation of the southern New Zealand landscape by tectonic uplift. This resulted in an incised valley through which flowed a braided river system. The particular disturbance regime in combination with a wet, fire-free environment, and a mean annual temperature of around $15-19{ }^{\circ} \mathrm{C}$, created a very biodiverse, predominantly rainforest vegetation. Retrophyllum was the dominant conifer and at times it was a prominent component of low-diversity vegetation patches in association with Myrtaceae. These low diversity communities may have been a response to repeated disturbance.

\section{ACKNOWLEDGEMENTS}

Special thanks to $\mathrm{Dr} \mathrm{T}$. Enright, for access to Dunstan Station, and to Dr. A. Kemp, CMM of the University of Queensland, and QUT, for access to SEM facilities. Access to the Queensland Herbarium continues to be much appreciated. The helpful comments of two anonymous reviewers improved the manuscript. I am indebted to the continuing support of Vicki Leung. 


\section{REFERENCES}

Bannister, J.M., Conran, J.G., and Lee, D.E. 2016. Life on the phylloplane: Eocene epiphyllous fungi from Pikopiko Fossil Forest, Southland, New Zealand. New Zealand Journal of Botany, 54:412-432. https://doi.org/10.1080/0028825x.2016.1208252

Blackburn, D.T. 1981. Tertiary megafossil flora of Maslin Bay, South Australia: Numerical taxonomic study of selected leaves. Alcheringa, 5:9-28. https://doi.org/10.1080/ 03115518108565430

Blume, 1850. Museum Botanicum Lugduno-Batavium, vol. 1. E. J. Brill, Leiden.

Brown, R. 1810. Prodromus Florae Novae Hollandiae et Insula Van-Diemen. J. Johnson and Co., London.

Burns, B.R. 1995. Environmental correlates of species richness at Waipoua Forest Sanctuary, New Zealand. New Zealand Journal of Ecology, 19:153-162. https://doi.org/10.1080/ $0028825 x .1996 .10412695$

Brown, R. 1814. Prodromous Florae Novae Hollandiae et Insulae Van-Diemen. Taylor, London.

Carpenter, R.J., Hill, R.S., and Jordan, G.J. 1994a. Cenozoic vegetation in Tasmania. macrofossil evidence, p.276-298. In Hill, R.S. (ed.), History of the Australian Vegetation. Cretaceous to Recent. Cambridge University Press, Cambridge.

Carpenter, R.J., Hill, R.S., and Scriven, L.J. 2006. Palmately lobed Proteaceae leaf fossils from the middle Eocene of South Australia. International Journal of Plant Science, 167:1049-1060. https://doi.org/10.1086/505537

Carpenter, R.J., Jordan, G.J., and Hill, R.S. 1994b. Banksiaephyllum taylorii (Proteaceae) from the Late Paleocene of New South Wales and its relevance to the origin of Australia's scleromorphic flora. Australian Systematic Botany, 7:385-392. https://doi.org/10.1071/ sb9940385

Carpenter, R.J. and Pole, M.S. 1995. Eocene plant fossils from the Lefroy and Cowan paleodrainages, Western Australia. Australian Systematic Botany, 8:1107-1154. https:// doi.org/10.1071/sb9951107

Christophel, D.C. and Blackburn, D.T. 1978. Tertiary megafossil flora of Maslin Bay, South Australia: a preliminary report. Alcheringa: An Australasian Journal of Palaeontology, 2:311319. https://doi.org/10.1080/03115517808527787

Conran, J.G., Bannister, J.M., Reichgelt, T., and Lee, D.E. 2016. Epiphyllous fungi and leaf physiognomy indicate an ever-wet humid mesothermal (subtropical) climate in the late Eocene of southern New Zealand. Palaeogeography, Palaeoclimatology, Palaeoecology, 452:1-10. https://doi.org/10.1016/j.palaeo.2016.03.032

Cookson, I.C. 1959. Fossil pollen grains of Nothofagus from Australia. Proceedings of the Royal Society of Victoria, 71:25-30.

Couper, R.A. 1953. Upper Mesozoic and Cainozoic spores and pollen grains from New Zealand. New Zealand Geological Survey Palaeontological Bulletin, 22:1-77.

Couper, R.A. 1960. New Zealand Mesozoic and Cainozoic plant microfossils. New Zealand Geological Survey Paleontological Bulletin, 32:1-88.

de Candolle, A.P. 1816. Essai sur les propriétés médicales des plantes. Crochard, Paris.

Dennstedt, A.W. 1818. Schluessel zum Hortus Indicus Malabaricus. Verlage des LandesIndustrie-Comptoirs, Weimar.

Dilcher, D.L. 1963. Eocene epiphyllous fungi. Science, 142:667-669. https://doi.org/10.1126/ science.142.3593.667

Dilcher, D.L. 1974. Approaches to the identification of angiosperm leaf remains. The Botanical Review, 40:1-157. https://doi.org/10.1007/bf02860067

Douglas, B.J. 1986. Lignite resources of Central Otago. New Zealand Energy Research and Development Committee Publication, P104:1-367.

Duigan, S.L. 1966. The nature and relationships of the Tertiary brown coal flora of the Yallourn area in Victoria, Australia. The Palaeobotanist, 14:191-200.

Duncan, R.P., Norton, D.A., and Woolmore, C.B. 1990. The lowland vegetation pattern, south Westland, New Zealand 2. Ohinemaka forest. New Zealand Journal of Botany, 28:131-140. https://doi.org/10.1080/0028825x.1990.10412353

Ellis, B., Daly, D.C., Hickey, L.J., Mitchell, J.V., Johnson, K.R., Wilf, P., and Wing, S.L. 2009. Manual of Leaf Architecture. Cornell University Press, Ithaca. 
Farjon, A. and Page, C.N., (eds.). 1999. Conifers. Status Survey and Conservation Action Plan. IUCN, IUCN/SSC Conifer Specialist Group, Gland and Cambridge.

Flower, B.P. and Kennett, J.P. 1994. The middle Miocene climatic transition: East Antarctic ice sheet development, deep ocean circulation and global carbon cycling. Palaeogeography, Palaeoclimatology, Palaeoecology, 108:537-555. https://doi.org/10.1016/00310182(94)90251-8

Greenwood, D.R. 1992. Taphonomic constraints on foliar physiognomic interpretations of Late Cretaceous and Tertiary palaeoclimates. Reviews of Palynology and Palaeobotany, 71:149190. https://doi.org/10.1016/0034-6667(92)90161-9

Hammer, Ø., Harper, D.A.T., and Ryan, P.D. 2001. PAST: Paleontological statistics software package for education and data analysis. Palaeontologia Electronica, 4(1):1-9. https://palaeo-electronica.org/2001_1/past/issue1_01.htm

Haston, E., Richardson, J.E., Stevens, P.F., Chase, M.W., and Harris, D.J. 2009. The Linear Angiosperm Phylogeny Group (LAPG) III: a linear sequence of the families in APG III. Botanical Journal of the Linnean Society, 161:128-131. https://doi.org/10.1111/j.10958339.2009.01000.x.

Hekel, H. 1972. Pollen and spore assemblages from Queensland Tertiary sediments. Publications of the Geological Survey of Queensland, 355:1-31.

Hickey, L.J. 1973. Classification of the architecture of dicotyledonous leaves. American Journal of Botany, 60:17-33. https://doi.org/10.2307/2441319

Hill, R.S. 1986. Lauraceous leaves from the Eocene of Nerriga, New South Wales. Alcheringa, 10:327-351. https://doi.org/10.1080/03115518608619144

Hill, R.S. and Brodribb, T. 1999. Southern conifers in time and space (Turner Review No. 2). Australian Journal of Botany, 47:639-696. https://doi.org/10.1071/bt98093

Hill, R.S. and Pole, M.S. 1994. Two new species of Pterostoma R.S. Hill from Cenozoic sediments in Australasia. Review of Palaeobotany and Palynology, 80:123-130. https:// doi.org/10.1016/0034-6667(94)90097-3

Holdgate, G.R., Cartwright, I., Blackburn, D.T., Wallace, M.M., Gallagher, S.J., Wagstaff, B.E., and Chung, L. 2007. The Middle Miocene Yallourn coal seam - The last coal in Australia. International Journal of Coal Geology, 70:95-115.

Hope, G. and Pask, J. 1998. Tropical vegetational change in the late Pleistocene of New Caledonia. Palaeogeography Palaeoclimatology Palaeoecology, 142:1-21. https://doi.org/10.1016/s0031-0182(97)00140-5

Issac, M.J., Lindqvist, J.K., and Pocknall, D.T. 1990. Geology and lignite resources of the East Southland Group, New Zealand. New Zealand Geological Survey Bulletin, 101:1-202.

Jaffré, T. 1995. Distribution and ecology of the conifers of New Caledonia, p. 171-196, In Hill, R. S. (ed.), Ecology of the Southern Conifers. Melbourne University Press, Melbourne.

Janzen, D.H. 1974. Tropical blackwater rivers, animals, and mast fruiting by the Dipterocarpaceae. Biotropica, 6:69-103. https://doi.org/10.2307/2989823

Johnson, L.A.S. 1980 Notes on Casuarinaceae. Telopea, 2:83-84.

Johnson, K.R. 2002. Megaflora of the Hell Creek and lower Fort Union formations in the western Dakotas; vegetational response to climate change, the Cretaceous-Tertiary boundary event, and rapid marine transgression. Special Paper Geological Society of America, 361:329-391. https://doi.org/10.1130/0-8137-2361-2.329

Jordan, G.J., Carpenter, R.J., and Hill, R.S. 1998. The macrofossil record of Proteaceae in Tasmania: a review with new species. Australian Systematic Botany, 11:465-501. https:// doi.org/10.1071/sb97020

Jussieu, A.L. 1789. Genera Plantarum. Herissant, Paris.

Kemp, E.M. 1978. Tertiary climatic evolution and vegetation history in the southeast Indian Ocean region. Palaeogeography, Palaeoclimatology, Palaeoecology, 24:169-208. https:// doi.org/10.1016/0031-0182(78)90042-1

Kuprianova, A.L. 1962: Palynological data and the systematics of the Fagales and Urticales, p. 17-25. In Sladkov, A.N. (ed.), For the Frst International Conference on Palynology: Reports of Soviet Palynologists (extended theses). U.S.S.R. Academy of Sciences, Moscow.

Landis, C.A., Campbell, H.J., Begg, J.G., Mildenhall, D.C., Paterson, A.M., and Trewick, S.A. 2008. The Waipounamu Erosion Surface: questioning the antiquity of the New Zealand land surface and terrestrial fauna and flora. Geological Magazine, 145:173-197. https://doi.org/ $10.1017 / \mathrm{s} 0016756807004268$ 
Leisman, G.A. 1986. Cryptocaryoxylon gippslandicum gen. et sp. nov., from the Tertiary of eastern Victoria. Alcheringa: An Australasian Journal of Palaeontology, 10: 225-234. https:// doi.org/10.1080/03115518608619157

Linnaeus, C. 1753. Species Plantarum, vol. 1. Laurentii Salvii, Stockholm.

Martius, C.F.P. 1824-1829. Nova Genera et Species Plantarum, quas in itinere per Brasiliam MDCCCXVII-MDCCCXX jussu et auspiciis Maximiliani Josephi I., Bavariae regis augustissimi instituto /collegit et descripsit C.F.P. de Martius., vol. 2. Impensis Auctoris, Munich.

McGlone, M.S. 1988. New Zealand, p. 557-602. In Huntley, B. and Webb, III T. (eds.), Vegetation History. New Zealand. Kluwers, Dordrecht. https://doi.org/10.1007/978-94-009-3081-0_15

McGlone, M.S., Richardson, S.J., and Jordan, G.J. 2010. Comparative biogeography of New Zealand trees: species richness, height, leaf traits and range sizes. New Zealand Journal of Ecology, 34:1-15.

Meylan, B.A. and Butterfield, B.G. 1978. The Structure of New Zealand Woods. DSIR, Wellington.

Mildenhall, D.C. 1989. Summary of the age and paleoecology of the Miocene Manuherikia Group, Central Otago, New Zealand. Journal of the Royal Society of New Zealand, 19:19-29. https://doi.org/10.1080/03036758.1989.10426452

Mildenhall, D.C. and Pocknall, D.T. 1989. Miocene-Pleistocene spores and pollen from Central Otago, South Island, New Zealand. New Zealand Geological Survey Palaeontological Bulletin, 59:1-128.

Miller, C. 2004. Floristics and species richness of floodplain forests, South Westland, New Zealand. New Zealand Journal of Botany, 42:847-860. https://doi.org/10.1080/ 0028825x.2004.9512934

Morgans, H.E.G., Beu, A.G., Cooper, E.M., Crouch, E.M., Hollis, C.J., Jones, C.M., Raine, J.I., Strong, C.P., Wilson, G.J., and Wilson, G.S. 2004. Paleogene (Dannevirke, Arnold and Landon Series), p. 125-163. In Cooper, R.A. (ed.), The New Zealand Geological Timescale. Paleogene (Dannevirke, Arnold and Landon Series). Institute of Geological and Nuclear Sciences Monograph, 22.

Müller, R.D., Sdrolias, M., Gaina, C., and Roest, W.R., 2008. Age, spreading rates and spreading asymmetry of the world's ocean crust. Geochemistry, Geophysics, Geosystems, 9:Q04006. https://doi.org/10.1029/2007gc001743

Nelson, C.S. and Cooke, P.J. 2001. History of oceanic front development in the New Zealand sector of the Southern Ocean during the Cenozoic-a synthesis. New Zealand Journal of Geology and Geophysics, 44:535-553. https://doi.org/10.1080/00288306.2001.9514954

Nix, H. 1982. Environmental determinants of biogeography and evolution in Terra Australis, $p$. 47-66. In Barker, W.R. and Greenslade, P.J.M. (eds.), Evolution of the Fauna and Flora of Arid Australia. Environmental Determinants of Biogeography and Evolution in Terra Australis. Peacock Publications, Fremville, South Australia.

Norton, D.A. and Leathwick, J.R. 1990. The lowland vegetation pattern, south Westland, New Zealand. 1. Saltwater Ecological Area. New Zealand Journal of Botany, 28:41-51. https:// doi.org/10.1080/0028825x.1990.10412342

Paijmans, K. (ed.) 1976. New Guinea Vegetation. CSIRO, Canberra.

Phipps, C.J. and Rember, W.C. 2004. Epiphyllous fungi from the Miocene of Clarkia, Idaho: reproductive structures. Review of Palaeobotany and Palynology, 129:67-79. https://doi.org/ 10.1016/j.revpalbo.2003.11.003

Pocknall, D.T. 1982. Pollen and spores from Blue Lake, St. Bathans (H41) and Harliwicks Lignite Pit, Roxburgh (G43), Central Otago, New Zealand. Palynology Section, NZGS, Lower Hutt, report.

Pocknall, D.T. 1989. Late Eocene to Early Miocene vegetation and climatic history of New Zealand. Journal of the Royal Society of New Zealand, 19:1-18. https://doi.org/10.1080/ 03036758.1989 .10426451

Pocknall, D.T. and Mildenhall, D.C. 1984. Late Oligocene-Early Miocene spores and pollen from Southland, New Zealand. New Zealand Geological Survey Paleontological Bulletin, 51:1-66.

Pole, M.S. 1991. A modified terminology for angiosperm leaf architecture. Journal of the Royal Society of New Zealand, 21:297-312. https://doi.org/10.1080/03036758.1991.10420828

Pole, M.S. 1992. Early Miocene flora of the Manuherikia Group, New Zealand. 2. Conifers. Journal of the Royal Society of New Zealand, 22:287-302. https://doi.org/10.1080/ 03036758.1992 .10420822 
Pole, M.S. 1993a. Early Miocene flora of the Manuherikia Group, New Zealand. 6. Lauraceae. Journal of the Royal Society of New Zealand, 23:303-312. https://doi.org/10.1080/ 03036758.1993.10721228

Pole, M.S. 1993b. Early Miocene flora of the Manuherikia Group, New Zealand. 8. Nothofagus. Journal of the Royal Society of New Zealand, 23:329-344. https://doi.org/10.1080/ 03036758.1993.10721230

Pole, M.S. 1993c. Early Miocene flora of the Manuherikia Group, New Zealand. 9. Miscellaneous leaves and reproductive structures. Journal of the Royal Society of New Zealand, 23:345391. https://doi.org/10.1080/03036758.1993.10721231

Pole, M.S. 1993d. Early Miocene flora of the Manuherikia Group, New Zealand. 5. Smilacaceae, Polygonaceae, Elaeocarpaceae. Journal of the Royal Society of New Zealand, 23:289-302. https://doi.org/10.1080/03036758.1993.10721227

Pole, M.S. 1993e. Early Miocene floras of the Manuherikia Group, New Zealand. 7. Myrtaceae, including Eucalyptus. Journal of the Royal Society of New Zealand, 23:313-328. https:// doi.org/10.1080/03036758.1993.10721229

Pole, M.S. 1993f. Early Miocene flora of the Manuherikia Group, New Zealand. 10. Paleoecology and stratigraphy. Journal of the Royal Society of New Zealand, 23:393-426. https://doi.org/ 10.1080/03036758.1993.10721232

Pole, M.S. 1997. Miocene conifers from the Manuherikia Group, New Zealand. Journal of the Royal Society of New Zealand, 27:355-370. https://doi.org/10.1080/03014223.1997.9517543

Pole, M.S. 1998. The Proteaceae record in New Zealand. Australian Systematic Botany, 11:343372. https://doi.org/10.1071/sb97019

Pole, M.S. 2003. New Zealand climate in the Neogene and implications for global atmospheric circulation. Palaeogeography, Palaeoclimatology, Palaeoecology, 193:269-284. https:// doi.org/10.1016/s0031-0182(03)00232-3

Pole, M. 2007a. Conifer and cycad distribution in the Miocene of southern New Zealand. Australian Journal of Botany, 55:143-164. https://doi.org/10.1071/bt06056

Pole, M. 2007b. Lauraceae macrofossils and dispersed cuticle from the Miocene of southern New Zealand. Palaeontologia Electronica, 10.1.3A:1-38. https://palaeo-electronica.org/ 2007 1/zealand/index.html

Pole, M. $2007 \mathrm{c}$. Monocot macrofossils from the Miocene of southern New Zealand. Palaeontologia Electronica, 10.3.15A:1-21. https://palaeo-electronica.org/2007_3/125/ index.html

Pole, M. 2008. Dispersed leaf cuticle from the Early Miocene of southern New Zealand. Palaeontologia Electronica 11.3.15A:1-117. https://palaeo-electronica.org/2008_3/153/ index.html

Pole, M. 2010. Was New Zealand a primary source for the New Caledonian flora? Alcheringa, 34:61-74. https://doi.org/10.1080/03115510903343477

Pole, M. 2014. The Miocene climate in New Zealand: Estimates from paleobotanical data. Palaeontologia Electronica 17.2.27A:1-79. https://doi.org/10.26879/436 https://palaeo-electronica.org/content/2014/780-miocene-climate-of-new-zealand.

Pole, M. and Douglas, B.J. 1998. A quantitative palynostratigraphy of the Miocene Manuherikia Group, New Zealand. Journal of the Royal Society of New Zealand, 28:405-420. https:// doi.org/10.1080/03014223.1998.9517572

Pole, M.S., Douglas, B.J., and Mason, G. 2003. The terrestrial Miocene biota of southern New Zealand. Journal of the Royal Society of New Zealand, 33:415-426. https://doi.org/10.1080/ 03014223.2003 .9517737

Pole, M., Dawson, J., and Denton, T. 2008. Fossil Myrtaceae from the Early Miocene of southern New Zealand. Australian Journal of Botany, 56:67-81. https://doi.org/10.1071/bt07032

Poole, I., Richter, H., and Francis, J.E. 2000. Gondwanan origins for Sassafras (Lauraceae): evidence from Late Cretaceous fossil wood of Antarctica. International Association of Wood Anatomists Journal, 21:463-475. https://doi.org/10.1163/22941932-90000262

Povey, D.A.R., Spicer, R.A., and England, P.C. 1994. Palaeobotanical investigation of early Tertiary palaeoelevations in northeastern Nevada: initial results. Review of Palaeobotany and Palynology, 81:1-10. https://doi.org/10.1016/0034-6667(94)90122-8

Raine, J.I. Mildenhall, D.C., and Kennedy, E.M. 2011. New Zealand fossil spores and pollen: an illustrated catalogue. 4th edition. GNS Science Miscellaneous Series no. 4. http:// data.gns.cri.nz/sporepollen/index.htm 
Reichgelt, T., Kennedy, E.M., Conran, J.G., Mildenhall, D.C., and Lee, D.E. 2015. The early Miocene paleolake Manuherikia: vegetation heterogeneity and warm-temperate to subtropical climate in southern New Zealand. Journal of Paleolimnology, 53:349-365. https:// doi.org/10.1007/s10933-015-9827-5

Romero, E.J. 1973. Polen fosil de "Nothofagus" ("Nothofagidites") del Cretacio y Paleoceno de Patagonia. Revista del Museo La Plata, 7:291-307.

Scriven, L.J. and Hill, R.S.1995. Macrofossil Casuarinaceae: their identification and the oldest macrofossil record, Gymnostoma antiquum sp. nov., from the Late Paleocene of New South Wales, Australia. Australian Systematic Botany, 8:1035-1053. https://doi.org/10.1071/ sb9951035

Stein, J, Craw, D., and Pope, J. 2011. Initial sedimentation and subsequent diagenesis in the Eastern Southland Lignite Basin, southern New Zealand. New Zealand Journal of Geology and Geophysics, 54:167-180. https://doi.org/10.1080/00288306.2010.515599

von Berchtold, B.V. and PresI, J.S. 1820. O Přirozenosti rostlin, aneb Rostlinář, 1:1-300

Wardle, P. 1974. The kahikatea (Dacrycarpus dacrydioides) forest of south Westland. Proceedings of the New Zealand Ecological Society, 21:62-71.

Wardle, P. 1977. Plant communities of Westland National Park (New Zealand) and neighbouring lowland and coastal areas. New Zealand Journal of Botany, 15:323-398. https://doi.org/ 10.1080/0028825x.1977.10432549

Wardle, P. 1980. Primary succession in Westland National Park and its vicinity, New Zealand. New Zealand Journal of Botany, 18:221-232. https://doi.org/10.1080/ 0028825x.1980.10426921

Webb, L.J. 1959. A physiognomic classification of Australian rainforests. Journal of Ecology, 47:551-570. https://doi.org/10.2307/2257290

Wehi, P.M. and Clarkson, B.D. 2007. Biological flora of New Zealand 10. Phormium tenax, harakeke, New Zealand flax. New Zealand Journal of Botany, 45:521-544. https://doi.org/ 10.1080/00288250709509737

Whitmore, T.C. 1986. Tropical Rain Forests of the Far East. English Language Book Society/ Oxford University Press, Oxford.

Wilf, P., Johnson, K.R., Cuneo, N.R., Smith, M.E., Singer, B.S., and Gandolfo, M.A. 2005. Eocene plant diversity at Laguna del Hunco and Rio Pichileufu, Patagonia, Argentina. The American Naturalist, 165:634-650. https://doi.org/10.1086/430055

Wolfe, J.A. 1979. Temperature parameters of humid to mesic forests of eastern Asia and their relation to forests of other regions of the northern Hemisphere and Australasia. US Geological Survey Professional Paper, 1106:1-37. https://doi.org/10.3133/pp1106

Wolfe, J.A., 1993. A method of obtaining climatic parameters from leaf assemblages. United States Geological Survey Bulletin, 2040:1-73. 